Travaux et documents du CIRAC

\title{
LES MÉDIAS À L'ÈRE DU NUMÉRIQUE
}

Réflexions franco-allemandes pour l'Europe

Sous la direction d'Isabelle BOURGEOIS

CIRAC 


\section{Les médias à l'ère du numérique}

Réflexions franco-allemandes pour l'Europe

Isabelle Bourgeois (dir.)

DOI : 10.4000/books.cirac. 622

Éditeur : IFAEE

Année d'édition : 2008

Date de mise en ligne : 13 décembre 2017

Collection : Travaux et documents du CIRAC

ISBN électronique : 9782905518590

\section{Doneratition}

http://books.openedition.org

\section{Édition imprimée}

ISBN : 9782905518361

Nombre de pages : 160

\section{Référence électronique}

BOURGEOIS, Isabelle (dir.). Les médias à l'ère du numérique : Réflexions franco-allemandes pour l'Europe. Nouvelle édition [en ligne]. Cergy-Pontoise : IFAEE, 2008 (généré le 02 octobre 2020). Disponible sur Internet : <http://books.openedition.org/cirac/622>. ISBN : 9782905518590. DOI : https://doi.org/ 10.4000/books.cirac.622.

(c) IFAEE, 2008

Conditions d'utilisation:

http://www.openedition.org/6540 
Travaux et documents du CIRAC

\section{LES MÉDIAS}

À L'ÈRE DU NUMÉRIQUE

Réflexions franco-allemandes

pour l'Europe

Sous la direction d'Isabelle BOURGEOIS 




\title{
LES MEDIAS
}

\section{A L'ERE DU NUMERIQUE}

\author{
Réflexions franco-allemandes \\ pour l'Europe
}

Sous la direction d'Isabelle BOURGEOIS

CIRAC

TRAVAUX ET DOCUMENTS DU CIRAC 


\section{Travaux et documents du CIRAC}

Collection dirigée par René Lasserre

(C) CIRAC, 2008

CIRAC, c/o Université de Cergy-Pontoise

33 Boulevard du Port - 95011 CERGY-PONTOISE CEDEX

ISBN 978-2-905518-36-1 


\section{AVANT-PROPOS}

Cet ouvrage retrace les débats entre experts et professionnels allemands, français et européens, réunis à Berlin, les 7 et 8 novembre 2006 dans le cadre d'un Dialogue francoallemand sur les médias, intitulé : "La concentration des médias à l'ère du numérique. Quelle prévoyance pour le marché et le citoyen?» (voir www.deutsch-franzoesischermediendialog.de). La problématique de ce Dialogue, organisé conjointement par l'instance de régulation de l'audiovisuel du Land de Thuringe, la Thüringer Landesmedienanstalt (TLM), son homologue du Land de Rhénanie du Nord-Westphalie, la Landesanstalt für Medien Nordrhein-Westfalen (LfM) et le Centre d'Information et de Recherche sur l'Allemagne Contemporaine (CIRAC), avait été conçue et développée conjointement par la TLM et le CIRAC.

Né à l'initiative de la société civile, ce Dialogue avait pour objectif, par-delà la confrontation des approches respectives des défis présentés par l'avènement de l'ère du numérique pour la régulation des médias, l'approche du marché et la défense du pluralisme, de favoriser une meilleure compréhension des contextes nationaux dans lesquels s'effectuent les choix institutionnels, politiques et réglementaires, comme des impératifs qui les guident. Car ce n'est que sur cette base que les acteurs politiques qui auront à définir les règles du nouveau 'modèle' médiatique de demain pourront développer conjointement des approches mieux coordonnées pour une action à l'échelon national, bilatéral et, surtout, communautaire. Inscrit dans le processus de transition vers l'économie du savoir et de la connaissance, le Dialogue franco-allemand sur les médias est amené à se prolonger.

Mes remerciements s'adressent à tous ceux qui, par leur engagement, leur curiosité des réalités du partenaire en Europe et leur grande disponibilité, ont contribué à ce que puisse émerger un dialogue profond et un échange fructueux - à commencer par les intervenants de ce Dialogue. Ils s'adressent, bien sûr, à l'Etat libre de Thuringe qui nous a chaleureusement accueillis dans sa représentation auprès du gouvernement fédéral à Berlin. Ils s'adressent particulièrement à Victor Henle, président de la TLM jusqu'en 2007, sans le partenariat engagé de qui ce Dialogue n'aurait pas pu voir le jour, et à son équipe, en tout premier lieu ses collaboratrices Doreen Schiller et Kathrin Wagner qui ont assumé l'organisation concrète de ces rencontres, soutenues du côté du CIRAC par Florentine Daudin, assistante du directeur. Enfin, mes remerciements tout particuliers s'adressent à Solène Hazouard, ingénieur d'études au CIRAC, sans le courage et la patience de qui la transcription des débats n'aurait pu se faire, et qui m'a secondée efficacement pour leur traduction.

Isabelle Bourgeois 



\title{
LES MEDIAS A L'ÈRE DU NUMÉRIQUE. REFLEXIONS FRANCO-ALLEMANDES POUR L'EUROPE
}

\author{
TABLE DES MATIÈRES
}

\author{
AVANT-PROPOS \\ Preface de Victor Henle \\ INTRODUCTION \\ Isabelle Bourgeois \\ La régulation entre deux logiques : prévoyance et libre concurrence

Debat $\mathbf{N}^{\circ} \mathbf{1}$

Dans la jungle des nouveaux services.

L'audiovisuel a-t-il vécu?

EXPOSE INTRODUCTIF

Isabelle Bourgeois

Des médias entre la loi et le marché.

Debat animé par Bénédicte de Peretti

INTERVENANTS : Francis Balle, Bernd Holznagel, Uwe Kammann

DEBAT $\mathbf{N}^{\circ} 2$

Nouveaux défis pour la régulation et le contrôle.

Quels niveaux et quelles instances?

EXPOSE INTRODUCTIF

Francis Balle

La genèse mouvementée de l'idée de régulation en France.

Debat animé par Hans-Jürgen Jakobs

INTERVENANTS : Dieter Dörr, Elisabeth Flüry-Hérard, Jasmin Kundan 


\section{DeBaT $\mathbf{N}^{\circ} 3$}

Parts de capital ou parts d'audience.

Ces critères sont-ils encore adaptés à l'ère du numérique ?

EXPOSE INTRODUCTIF

Isabell Hülsen

De l'incompatibilité entre parts de marché et parts d'audience

DÉBAT animé par Isabelle Bourgeois

INTERVENANTS : Alain Lancelot, Jürgen Heinrich, Dieter Schmidtchen

\section{DEBAT $N^{\circ} 4$}

Segmentation de l'offre, individualisation de la réception, atomisation de la société.

Quelles bases de légitimité pour une régulation des médias?....

EXPOSE INTRODUCTIF

Jean-Louis Missika

Heurs et malheurs de la révolution numérique.

DebAT animé par Andreas Stopp

INTERVENANTS : Jean-Louis Missika, Jacques Rigaud, Norbert Schneider

\section{DeBAT ${ }^{\circ} 5$}

Les entreprises s'européanisent.

Faut-il un contrôle des concentrations

pour les médias à l'échelon européen?

EXPOSE INTRODUCTIF

Thomas Kleist

En l'absence d'un espace public européen, l'échelon national

reste le cadre pertinent de la garantie du pluralisme.

DeBAT animé par Joseph Lorent

INTERVEnANTS : Olaf Christiansen, Peter Eberl, Werner Schwaderlapp

PostFace de Victor Henle

Construire la démocratie de la communication

LES INTERVENANTS 139

INDICATIONS BIBLIOGRAPHIQUES 147 


\section{Preface}

La concentration des médias s'est fortement intensifiée ces dernières années. Dans les seuls mois précédant ce Dialogue franco-allemand sur les médias, l'Office fédéral des cartels avait été amené à émettre publiquement de sérieuses réserves quant à un projet de fusion. Il aurait réuni l'opérateur de télévision ProSiebenSat.1 et le groupe d'édition de presse Axel Springer. Ce projet de fusion, abandonné en février 2006, avait non seulement été au centre des préoccupations des Landesmedienanstalten, les autorités allemandes de régulation de l'audiovisuel, ou des diverses institutions en charge de l'évaluation du degré de concentration des médias, il avait aussi été amplement débattu dans l'espace public. Pendant près d'un an, une question a préoccupé l'opinion allemande : comment limiter les risques de voir un acteur occuper une position dominante sur les marchés des médias et développer un pouvoir d'influence démesuré sur l'opinion publique?

Pas plus tard que la veille de ce Dialogue, la Fondation Friedrich Ebert avait réuni à Berlin des experts pour discuter du "contrôle des opinions en position dominante à l'heure de la convergence ${ }^{1}-$ une thématique proche de celle que nous abordons aujourd'hui. Il s'agissait de définir des règles pour la démocratie et l'économie dans l'ère du numérique, et d'explorer les causes et conséquences de la concentration des médias.

En France, en décembre 2005, la commission présidée par Alain Lancelot avait soumis au Premier ministre une brillante analyse des questions juridiques et économiques soulevées par la concentration des médias. Elle était parvenue à la conclusion que, en France, la concentration des médias n'avait pas encore atteint un degré alarmant. Néanmoins elle précisait que de nombreuses modifications devaient être apportées à la réglementation en vigueur, notamment une réforme du droit commun, du droit de la procédure et une restructuration des compétences institutionnelles. Je vous recommande la lecture de son analyse et de ses propositions de réforme, qui sont lourdes d'enseignement également pour l'approche allemande.

Nous entrons dans l'ère de la communication numérique. Elle défie l'organisation fédérale du système allemand des médias, en forçant un changement de paradigme dans le domaine de la régulation des médias. Les barrières que nous avons instaurées contre la concentration des médias dans le monde de l'analogique ne sont plus adaptées pour l'endiguer dans l'univers du numérique.

Malgré les différences structurelles entre la France et l'Allemagne, les mutations en cours se traduisent par des effets présentant de grandes similitudes. Il y a peu encore, la concentration des médias revêtait un profil classique que Jean-Marie Messier, alors

\footnotetext{
${ }^{1}$ « Kontrolle von Meinungsmacht in Zeiten der Konvergenz - Demokratie und Wirtschaft brauchen klare Regeln », Medien-Fachkonferenz der Friedrich-Ebert-Stiftung, Berlin, 6 novembre 2006.
} 
président du groupe Vivendi Universal qu'il avait constitué, avait décrit ainsi en 1997 dans une interview accordée au quotidien Le Monde : "Il y aura bientôt un seul point d'entrée dans la maison pour l'image, la voix, le multimédia et l'accès à l'Internet». Puis il en tirait la conclusion suivante : "Cette perspective m'a amené à conclure qu'il faut être capable, pour conserver les marges, de maîtriser toute la chaîne contenuproduction-diffusion et lien avec l'abonné ». Leo Kirch ne s'était pas exprimé en ces termes mais il avait agi en conséquence.

Les maîtres de ce monde analogique des médias étaient et sont partiellement encore les grands groupes : Bertelsmann, Berlusconi, Pearson, Murdoch, Prisa et Vivendi Universal. Dans leurs sociétés, ils produisent tout ce que demande le consommateur des médias : journaux, magazines, livres, cinéma, musique, jeux, programmes radio et télévisés, sans oublier l'offre d'Internet. Ils ambitionnent de devenir l'interlocuteur unique du consommateur des médias.

\section{Les 'seigneurs des réseaux' seront-ils les maîtres de demain ?}

De nouveaux maîtres sont apparus dans le combat pour la suprématie dans le salon numérique du consommateur: les 'seigneurs des réseaux' que sont les groupes de télécommunications. Ils ne se contentent plus de leur rôle historique d'opérateurs de réseaux, c'est-à-dire de prestataires chargés de l'emballage technique des contenus ou de transporteurs acheminant les contenus vers le salon et, depuis peu, vers le téléphone portable du consommateur. Car eux aussi sont soumis maintenant aux rudes lois de l'économie qui les contraignent à épuiser l'ensemble de la chaîne de création de valeur, une tendance qui accélère le mouvement de concentration sur le marché des médias.

Les opérateurs de réseau, qui en Allemagne desservent la moitié des 37,5 millions de foyers télévisés, s'emparent du métier d'opérateur de plateforme qu'assuraient auparavant les sociétés de programmes. Ainsi par exemple, Unity Media, qui est l'opérateur des réseaux câblés de Rhénanie du Nord-Westphalie et de Hesse, vient de s'adjoindre deux rôles totalement nouveaux pour accroître l'attractivité de l'abonnement au réseau : rendre la réception câblée plus attrayante. Il a acheté à prix d'or les droits de retransmission des contenus TV les plus prisés en Allemagne : les matches de football de la Bundesliga. Et, fort de cette matière première inestimable, il assemble maintenant sa propre chaîne.

Le changement réside dans le fait que les opérateurs de réseaux câblés tendent à se positionner en concurrents de leurs propres clients, alors qu'auparavant, ils se contentaient de se concurrencer entre eux. Si cette convergence des rôles d'éditeur de contenus et de gestionnaire de réseau est nouvelle en Allemagne, elle est déjà bien établie en France, où un groupe de BTP détient à la fois la première chaîne privée TF1 et Bouygues Télécom, sa filiale de téléphonie mobile. Cette même convergence caractérise Vivendi Universal qui, outre ses chaînes, détient l'opérateur de téléphonie mobile SFR.

On assiste ainsi à une 'renaissance' de la tendance à l'intégration verticale. Après la chute de 'l'empire Kirch' au printemps 2002, nous avons cru en Allemagne que cette forme de concentration appartenait au passé. Mais maintenant que les opérateurs de réseau se mettent à créer leurs propres plateformes pour commercialiser des programmes qu'ils éditent eux-mêmes et à les distribuer de surcrôt à l'aide de technologies propriétaires, les problématiques du contrôle des concentrations et de la garantie du pluralisme 
se sont déplacées vers un autre aspect : comment garantir les mêmes chances d'accès au marché aux acteurs tiers? Mais dans le même temps, force est de constater aussi qu'avec ces nouveaux acteurs est apparu un nouveau type de concurrence sur les réseaux de distribution des contenus audiovisuels. Ceux-ci se sont élargis aux réseaux à haut débit du VDSL dans le domaine filaire, ou, dans le hertzien, aux réseaux de télévision numérique mobile (DVB-H).

La question qui se pose dès lors est de savoir si cette nouvelle forme que prend la concurrence n'est pas de nature aussi à désamorcer dans une certaine mesure la problématique du contrôle des concentrations spécifique au domaine des médias. Elle nous amène en tout cas à nous demander si nous n'allons pas, à l'avenir, pouvoir renoncer au moins partiellement à prendre des mesures prophylactiques. Y renoncer totalement ne semble guère possible, la raison économique l'interdit. Rupert Murdoch, en maître de l'art, avait un jour décrit ainsi cette raison. "Je déteste les monopoles », avait-il dit, avant de poursuivre : "sauf si j'en avais un ».

Sur la voie qui mène aux nouvelles configurations des réseaux audiovisuels, Deutsche Telekom est revenue récemment à un statu quo ante. Sous la contrainte de la Commission européenne, l'opérateur a dû vendre ses réseaux câblés. Mais il s'est mis à en construire de nouveaux, à très haut débit, en technologie VDSL, pour recréer ses propres vecteurs de distribution pour les contenus audiovisuels entre autres.

Ces opérateurs de télécommunications disposent d'énormes ressources financières. Elles leur donnent une latitude d'action bien supérieure à celle des traditionnelles sociétés de programmes. A l'instar d'Unity Media, elles s'attachent leurs clients grâce à une offre exclusive et globale de services de communication, ce triple play qui ouvre accès à tous les services (e-mails, téléphonie, accès Internet, radio, télévision) mais qui, rendant plus difficile pour le consommateur toute tentative de changer de fournisseur, en fait un client captif. Cette stratégie qui crée de nouvelles positions de pouvoir sur le marché finira par aboutir à une éviction de la concurrence.

\section{Nos sociétés subissent de profondes transformations}

Des deux côtés du Rhin, de profondes mutations sont en cours. Les frontières sectorielles s'estompent, les concepts établis perdent de leur consistance, communication individuelle et communication de masse tendent à s'interpénétrer... Ces évolutions mettent à mal les seuils érigés pour contenir le pouvoir des médias, qu'il s'agisse du modèle allemand des parts d'audience ou du modèle français des parts de capital. Centrer le contrôle des concentrations sur l'éditeur, comme c'était le cas jusqu'à présent, ne suffira bientôt plus pour contenir l'émergence d'opinions prédominantes. Ce sont aujourd'hui les opérateurs de réseau qui exercent le pouvoir de décision, ce sont eux qui décident si les contenus offerts par celui qui 'contrôle' l'opinion par sa position prédominante peut les diffuser aux consommateurs via leurs réseaux de distribution.

La numérisation a déjà profondément modifié la donne, même si nul ne sait encore quel univers naîtra de la formidable dynamique de changement actuellement à l'oeuvre. Pour l'heure, c'est aux opérateurs de réseau et aux investisseurs financiers à qui ils appartiennent que revient le rôle central. Quand la presse relate par exemple qu'un investisseur comme KKR, qui n'est pas seulement un «prédateur » comme on l'affirme trop hâtivement, s'apprêterait à proposer quelque 40 milliards d'euros pour racheter Vivendi 
Universal, alors on comprend mieux où se situent les véritables enjeux de pouvoir. Inéluctablement, ces mutations vont décider du destin de l'audiovisuel qui joue encore - mais pour combien de temps ? - un rôle privilégié dans nos sociétés, protégé en France comme en Allemagne par les garanties institutionnelles que lui confère sa mission spécifique.

Je souhaite que ces deux journées de Dialogue franco-allemand sur les médias nous permettent de confronter nos expériences respectives des profonds changements que connaissent nos sociétés en entrant dans l'ère de l'information et l'économie de la connaissance. Puissions-nous en sortir mieux armés encore pour relever ensemble les défis que représentent ces mutations pour nos systèmes médiatiques, bien sûr, mais bien plus encore pour nos modèles économiques et démocratiques. Et puissions-nous approfondir nos échanges pour ainsi mieux répondre aux enjeux de la communication dans une Union européenne à l'aube de la société du savoir.

Victor Henle

Président de la Landesmedienanstalt Thuringen (TLM) 
INTRODUCTION 



\title{
La régulation entre deux logiques : prévoyance et libre concurrence
}

\author{
Isabelle Bourgeois
}

\begin{abstract}
«Tout progrès technique préfigurant l'avenir de la communication de masse soulève des questions nouvelles qui exigent une réponse. Les leçons tirées du passé peuvent nous aider à maîtriser l'avenir, même si le rythme des innovations technologiques s'accélère continûment. Car quoi qu'il advienne, c'est à l'Homme qu'il revient au bout du compte de maîtriser le flux d'information libéré par la technologie » (Hans Bausch, 1980). ${ }^{2}$
\end{abstract}

Voici ce qu'écrivait voici près de trente ans Hans Bausch, l'un des pères fondateurs de la radiodiffusion allemande de l'après-guerre. Sa pensée est aujourd'hui plus pertinente et actuelle que jamais. A l'époque, on ne savait pourtant encore rien d'Internet, de la TNT, du triple play ou de la télévision mobile. Mais en France comme en Allemagne se développaient ces 'nouveaux médias' dont on espérait et redoutait en même temps de profonds bouleversements.

Depuis toujours, les mutations technologiques et l'essor du marché...

Tout comme aujourd'hui, c'est l'arrivée de nouvelles technologies qui fut le levier du changement : le progrès lié à la compression des données a accéléré la généralisation des télécopieurs, permis l'essor des services de télétexte ou de vidéotexte et favorisé le développement de nouveaux standards préfigurant la TV haute définition. De nouveaux modes de transmission émergeaient : en France, les radios privées s'emparent de la bande FM, créant une situation nouvelle qui forcera la désétatisation et la libéralisation de l'audiovisuel.

France et Allemagne développent à cette époque les infrastructures du câble, suivant toutefois deux approches différentes. En France, on mise sur la fibre optique et sur un nouveau business modèle (la TV à péage) reposant sur les nouvelles technologies de cryptage. Certes, la fibre optique n'en était encore qu'à la phase du prototype, ce qui a retardé l'expansion prévue, mais les bases économiques étaient jetées pour les offreurs de plateformes à venir. En Allemagne, on préfère miser sur le cuivre coaxial, et le réseau câblé est conçu pour la seule redistribution d'une offre de télévision gratuite élargie (extension des supports et capacités).

\footnotetext{
${ }^{2}$ Hans Bausch (ed), Rundfunk in Deutschland, texte figurant en exergue au vol. 3, deuxième partie (Rundfunkpolitik nach 1945), Munich, 1980.
} 
Dans les deux pays se développent en parallèle les modes de transmission hertziens grâce aux satellites destinés à la réception directe. La France et l'Allemagne mettent en place conjointement le système de satellites jumeaux TV SAT/TDF. Bien que, suite à une série d'avaries, le projet ait été abandonné, il a modifié la donne du paysage audiovisuel allemand, puisqu'il a contraint les Länder à s'accorder sur l'ouverture du marché à des opérateurs privés. C'est ainsi que furent posés les jalons de la signature par les Länder, en 1986, du Contrat d'Etat sur le Nouvel ordre de la radiodiffusion (Staatsvertrag zur Neuordnung des Rundfunkwesens) qui est le texte fondateur du système dual actuel de l'audiovisuel.

Au cours de l'été 1989 est lancé le premier satellite Astra. Quelques mois plus tard, la chute du Mur entraîne la subite extension du marché allemand. Les positions établies sur les divers segments du marché des médias s'en trouvent bousculées, forçant une croissance par-delà les frontières établies qui séparaient la presse de l'audiovisuel, et le marché se restructure dans le contexte de la croissance exponentielle de la demande face à une offre étendue, presque exclusivement en matière de télévision gratuite.

En France aussi, le lancement des satellites Astra se traduira quelques années plus tard par un immense élargissement de l'offre, mais seulement au niveau de la TV à péage. Les bouquets satellites TPS et CanalSat (qui fusionneront en 2006) fournissent une offre complémentaire à celle proposée par le câble. Aujourd'hui, l'extension rapide des capacités hertziennes terrestres de la TNT devrait avoir en France le même effet que le lancement d'Astra dans l'Allemagne des années 1990, c'est-à-dire un rapide élargissement du marché.

La mise en œuvre de nouveaux moyens de diffusion a réduit dans les deux pays la rareté des fréquences et a ainsi mené à une plus grande différenciation de l'offre. Le marché publicitaire s'est accru et fragmenté en même temps que se démultipliaient les supports et que ceux-ci se segmentaient. Sur le segment de l'audiovisuel s'est établi, parallèlement au financement classique par la publicité, un nouveau business modèle, hérité de la presse : celui de la TV par abonnement. En d'autres termes : ces évolutions ont créé une nouvelle donne, transformant le paysage audiovisuel en un marché.

... défient le législateur chargé de réguler les médias...

Elles ont placé les législateurs face à un nouveau défi ; en réponse, ils ont libéralisé la radiodiffusion ou, plus précisément, ils ont autorisé de nouveaux opérateurs privés à entrer sur le marché, parallèlement au service public. Au milieu des années 1990 se sont ainsi consolidées en France comme en Allemagne les structures de la régulation des médias telles qu'elles prévalent toujours dans leurs grandes lignes.

Depuis, les deux pays se caractérisent par un système dual au sein duquel les opérateurs privés sont soumis au régime de l'autorisation. L'obtention d'une licence est liée à certaines obligations de service public dont l'objectif est de garantir une relative complémentarité dans l'offre globale de radiodiffusion. La finalité de ce régime est la garantie du pluralisme des opinions, ou plus exactement : la garantie de la diversité des opinions diffusées par les médias audiovisuels ; celle-ci devant, dans la mesure du possible, refléter en totalité le spectre des opinions existant dans la société.

Cette approche de la régulation, qui trouve son fondement dans le droit constitutionnel, repose sur la représentation d'un récepteur passif. Et elle se fonde sur une définition 
de la radiodiffusion née au début des années 1920 - une définition forgée pour la transmission/diffusion du signal/message d'un point vers la masse au moyen d'ondes électromagnétiques : donc pour le broadcasting, en opposition à la communication point à point des télécommunications en plein développement à la même époque.

... et remettent en question aujourd'hui le statut d'exception dont jouit l'audiovisuel

Cette définition de l'audiovisuel, née alors du besoin de distinguer deux applications distinctes de la même technologie, se maintient jusqu'à aujourd'hui, constituant toujours la justification d'une régulation sectorielle de l'audiovisuel qui, en Allemagne, relève de la société civile, en France, de l'Etat. Cette approche confère à la radiodiffusion un statut particulier ; or ce faisant, elle ne considère qu'un seul segment de l'offre globale des médias de masse.

Pendant que s'établissaient ainsi dans les deux pays les contours de la réglementation/régulation de l'audiovisuel, le marché continuait de se développer, donnant naissance à des structures oligopolistiques qui perdurent jusqu'à aujourd'hui. Mais alors qu'en Allemagne, c'est presque exclusivement le capital des médias eux-mêmes qui finance la croissance du marché, en France, c'est principalement le capital industriel et, plus précisément, celui des industries de réseaux.

Après cette phase de croissance à l'intérieur des frontières nationales respectives, une 'ronde des alliances' commence à se former en Europe et dans le monde. La libéralisation des marchés de la radiodiffusion avait favorisé l'émergence de nouveaux acteurs qui aspirent désormais à se développer au-delà des frontières nationales ainsi que sur des segments de marché voisins. La libéralisation des marchés des télécommunications, qui intervient à la même époque, entretient un contexte favorable à une effervescence qui se nourrit aussi de nouvelles perspectives technologiques. C'est durant cette phase, caractérisée par une série de fusions ou de projets spectaculaires au milieu des années 1990, qu'apparaît pour la première fois le concept de convergence que résume à merveille ce qui fut alors la devise d'un Jean-Marie Messier à la tête du groupe qu'il voulait hisser au rang de 'global player' (autre formule caractéristique de cette époque) : «des tuyaux aux contenus ».

C'est au plus tard à partir de ces années que se pose la question brûlante d'une régulation du marché de la radiodiffusion, aussi bien au niveau national qu'à l'échelon européen. Car, parallèlement, le processus d'intégration européenne s'était accéléré.

Or une telle régulation était et demeure une entreprise délicate du fait de la double exception culturelle de l'audiovisuel. Bien qu'il fasse partie du secteur des services et plus précisément des services d'intérêt général, il n'est pas considéré comme partie intégrante de l'économie, ni au niveau européen, ni au niveau national. S'ajoute à cela le fait que se cristallise sur l'audiovisuel la défense d'une conception du principe de subsidiarité qui amène les Etats membres à se crisper sur leurs approches nationales respectives. La culture dans son sens le plus large est en effet l'un des derniers domaines de souveraineté des Etats membres dans l'Union.

L'entreprise est rendue encore plus délicate par une contradiction foncière : de jure, l'audiovisuel n'est pas considéré comme un marché bien qu'il soit de facto un marché bien réel. En tant que tel, le marché, et les entreprises qui en sont les acteurs, est bien entendu soumis au droit de la concurrence. Seulement, cette situation soulève la 
question des critères adéquats pour considérer l'état de la concurrence, à savoir celle de la compatibilité entre deux concepts issus de sources de droit distinctes: "opinion prédominante » d'une part, "position dominante » sur le marché, de l'autre.

Cette question en soulève une autre, directement liée : celle de la compétence des institutions respectives chargées du contrôle de la concentration - d'un côté, Office fédéral des Cartels (Bundeskartellam)t, Conseil de la concurrence ou Direction générale de la concurrence; de l'autre, instances de régulation sectorielles nationales ou Viviane Reding, Commissaire européen en charge de la société de l'information et des médias. Une véritable quadrature du cercle...

Quelle finalité pour la régulation ? Comment concilier libre concurrence et prévoyance ?

Or pendant que législateurs et instances de régulation se concentrent ainsi sur la définition du marché et son appréhension réglementaire, le progrès technologique se poursuit : la numérisation s'accélère. L'évolution coïncide dans le temps avec la libéralisation des marchés de la télécommunication en Europe. Ce domaine est lui aussi soumis à une régulation sectorielle mais, à la différence de son homologue pour la radiodiffusion, cette régulation vise a priori la constitution d'un marché fonctionnel établi sur la concurrence des acteurs.

Cette approche place au cœur du dispositif de régulation le consommateur afin que celui-ci dispose de services abordables et accessibles à tous. Mais ce faisant, et du fait qu'elle se justifie par le processus de libéralisation, c'est-à-dire une phase préalable à l'émergence d'un marché, elle constitue une entorse au droit commun de la concentration.

Ulf Böge, président de l'Office fédéral des Cartels, décrit ainsi la finalité du droit allemand de la concurrence : il "vise à protéger la concurrence 'en tant qu'institution', essentiellement pour garantir et/ou donner aux sujets économiques la plus grande liberté individuelle d'action possible. Cela va bien au-delà de la simple protection du consommateur ou, pour le dire autrement : la finalité explicite du droit allemand de la concurrence n'est pas de créer ou préserver une sorte de 'welfare' pour le consommateur ». Toutefois, le droit allemand "repose sur l'hypothèse que la concurrence contribue à générer les conditions les plus favorables au consommateur ». ${ }^{3}$

Par contraste s'éclaire mieux ainsi l'approche de la régulation spécifique à la radiodiffusion : elle repose sur une logique de répartition, légitimée par une pénurie de l'approvisionnement, elle-même induite à l'origine par la rareté des fréquences.

Or cette rareté n'existe plus vraiment. Du côté de l'offre des contenus domine aujourd'hui le principe du kiosque - celui qui a de tout temps caractérisé le marché de la presse. Malgré une forte concentration du côté des opérateurs, la diversité des opinions n'a jamais été mise en danger dans le domaine de la presse. Car c'est le consommateur, en tant qu'acheteur d'un produit, abonné d'une prestation ou en tant que contact publicitaire, qui décide en dernier ressort de l'évolution du marché. Le lecteur d'un journal est considéré comme un client/usager actif et responsable. Ses choix ne requièrent nulle assistance. Et la prévoyance se limite à la garantie de la concurrence sur le marché de la

\footnotetext{
${ }^{3}$ Ulf Böge, «Effizienz und Wettbewerb aus Sicht des Bundeskartellamts », in Peter Oberender (ed), Effizienz und Wettbewerb, Berlin, 2005. NB. : le $1^{\text {er }}$ avril 2007, Bernhard Heitzer a succédé à U. Böge à la tête du Bundeskartellamt.
} 
presse écrite. Dans le domaine des télécommunications s'esquisse une approche comparable.

Un prisme de cinq approches pour prendre en compte la complexité des interrogations

Le statut particulier de la radiodiffusion perdure, quant à lui, alors même que l'offre globale de contenus médiatiques est aujourd'hui immense et diversifiée. La multiplication de l'offre de services interactifs soulève la question de la concentration sur une vision purement passive du consommateur des médias. Se justifie-t-elle toujours ? C'est là la première problématique qu'aborde ce Dialogue franco-allemand sur les médias.

La deuxième s'articule autour de la dichotomie entre d'un côté marchés et services convergents et, de l'autre, approches de régulation divergentes. L'existence parallèle de régimes juridiques et d'instances de régulation sectoriels se justifie-t-elle toujours ? Il est probablement plus facile d'appréhender cette problématique en France qu'en Allemagne où la Loi fondamentale institue une sorte de 'répartition des pouvoirs' entre les sources du droit et les échelons de compétences législatives s'appliquant aux infrastructures (droit commun, échelon fédéral) et aux contenus (droit constitutionnel, souveraineté des Länder)...

Il est incontestable que le marché des médias doit être régulé pour offrir à tous les acteurs économiques (du fournisseur au consommateur) "la plus grande liberté individuelle d'action possible ». Reste à savoir comment, avec quels instruments et selon quels critères ? De même, un système de radiodiffusion dual est-il encore justifié ? Et comment légitimer aujourd'hui le statut spécifique d'un service public de l'audiovisuel ? Voilà la troisième problématique abordée.

Le quatrième débat opère un changement de perspective. Si on considère les acteurs économiques en tant que citoyens, alors se pose la question des effets sur la société d'une offre de plus en plus fragmentée et de comportements de réception de plus en plus individualisés. Jean-Louis Missika prévoit «la fin de la télévision $»^{4}$ en tant que média d'intégration. Une nouvelle atomisation de la société menace-t-elle ? Ou voyons-nous émerger de nouvelles formes de participation qui exigent que chacun dispose des compétences stratégiques à l'ère de la société du savoir ? Dans ce cas, que faut-il réguler et comment le faire ?

Cinquième problématique. Le marché intérieur a depuis longtemps aboli les frontières nationales, sauf dans le domaine des services, sans parler de l'audiovisuel, dont les cadres juridiques respectifs restent fortement nationaux. Dans la pratique, il s'avère extrêmement difficile pour un opérateur de radiodiffusion de s'implanter dans un pays voisin. Il n'en va pas de même lorsqu' on considère le marché intérieur comme une entité au sein duquel règne une demande multiforme. Les entreprises mettent alors en place des coopérations, fusionnent de temps à autre, ou, le plus généralement, proposent aux utilisateurs des produits adaptés, déclinés sur-mesure selon les goûts nationaux respectifs. On voit ainsi émerger petit à petit un marché de dimension européenne au niveau d'une offre ciblée sur la demande commune à des groupes ou milieux se caractérisant par des traits socioculturels similaires, indifféremment des frontières nationales. Mais le simple constat de l'existence de ces similitudes de valeurs dans des groupes très

${ }^{4}$ Jean-Louis Missika, La fin de la télévision, Paris, 2006. 
fragmentés permet-elle d'en conclure qu'elles préfigurent un espace public européen ? Comment donc le droit devrait-il prendre en considération ces évolutions ? Il va de soi que nous avons besoin de règles du jeu. Mais quelles devraient-elles être ? Et quelles devraient être les instances compétentes?

En d'autres termes: Quelle prévoyance pour le marché et le citoyen ? Voilà la question centrale de ce Dialogue franco-allemand sur les médias. L'approche en termes de welfare est-elle toujours justifiée ? Et si oui, quelles devraient être sa nature et sa configuration en droit? Si la question se pose pareillement dans nos deux pays, ses termes diffèrent.

Confrontons donc les approches respectives et laissons-nous guider dans nos débats par les réflexions de Hans Bausch : «quoi qu'il advienne, c'est à l'Homme qu'il revient au bout du compte de maîtriser le flux d'information libéré par la technologie »... 


\section{Debat $\mathbf{N}^{\circ} \mathbf{1}$}

\section{DANS LA JUNGLE DES NOUVEAUX SERVICES.}

L’audiovisuel a-t-il vécu ? 



\title{
DEBAT $\mathrm{N}^{\circ} \mathbf{1}$
}

\section{DANS LA JUNGLE DES NOUVEAUX SERVICES. L'audiovisuel a-t-il vécu?}

\author{
EXPOSE INTRODUCTIF : Isabelle Bourgeois (CIRAC) \\ INTERVENANTS : \\ Francis Balle (IFP, Université Paris 2) \\ Bernd Holznagel (Université de Münster) \\ Uwe Kammann (Adolf-Grimme-Institut)
}

Débat animé par Bénédicte de Peretti (La Tribune)

\begin{abstract}
L'émergence de nouveaux acteurs, la multiplication des nouveaux supports et services de communication numériques, le changement du comportement des consommateurs amènent à repenser une régulation de l'audiovisuel qui trouve ses fondements dans la genèse de la radio au début du XXe siècle.

Depuis, sa légitimation repose sur l'idée d'un consommateur passif, exposé d'une manière quasi captive à une offre point à multipoint (broadcasting) réduite du fait de la rareté des infrastructures (fréquences). Or à l'ère du numérique, le consommateur et citoyen tend à développer une demande différenciée et, surtout, active.

Cette évolution plaide pour un changement de paradigme dans la régulation. Ne conviendrait-il pas de la réorienter vers les aspects de demande et d'y intégrer l'ensemble du champ des médias ? La multiplication de l'offre point à point rend de plus en plus délicate la délimitation du périmètre de l'audiovisuel, soulevant la question de la justification de son statut spécifique.

Comment garantir dorénavant le pluralisme d'une offre médiatique certes augmentée, mais avant tout en pleine fragmentation? Comment justifier un service public de l'audiovisuel dans un marché caractérisé par une abondance de l'offre, le plus souvent payante?

La régulation ne devrait-elle pas dès lors se concentrer sur les nouveaux gate keepers que sont les offreurs de plateformes ou de moteurs de recherche? Comment, dans ce contexte, garantir une sorte de "service universel " pour réduire les risques d'exclusion et préserver le lien social?
\end{abstract}





\section{EXPOSE INTRODUCTIF}

\section{Des médias entre la loi et le marché}

\section{Isabelle Bourgeois}

"Aujourd'hui, la convergence numérique entraîne... petit à petit une confusion des métiers... qui risque de faire perdre son autonomie à la télévision », écrit Jean-Louis Missika dans son essai intitulé "La fin de la télévision » et paru en mars 2006. Depuis une décennie, les métiers d'éditeur, de diffuseur, de fournisseur d'accès se recomposent, cette tendance remettant en question le rôle fédérateur qui était dévolu depuis un demi-siècle au seul grand média capable de rassembler les citoyens-consommateurs et de ce fait indispensable pour structurer l'espace public et créer le lien social : la télévision.

Or si cette tendance est universelle, elle se traduit de diverses manières dans nos deux pays, pour des raisons liées à la structuration des marchés médiatiques, à l'organisation de la concurrence entre audiovisuel public et privé, ainsi qu'à des modes de régulation déterminés à la fois par les sources de droit applicables à ces activités et à la configuration des systèmes politiques. Ces divergences systémiques sont si profondes que la France et l'Allemagne peuvent être considérées comme deux 'modèles' aux antipodes l'un de l'autre au sein de l'Union européenne, ceux des autres Etats membres ne présentant que des variantes plus ou moins métissées.

\section{Deux marchés médiatiques présentant des structures différentes}

France et Allemagne se distinguent d'une part, au sein de l'UE, par la taille de leurs marchés médiatiques. Depuis longtemps, le marché publicitaire allemand 'pèse' le double de son équivalent français. La répartition du gâteau diffère également : en Allemagne, malgré une tendance à la baisse, la presse quotidienne se taille toujours la part du lion, suivie de loin par la télévision, la radio se contentant de 'rogatons'. En France à l'opposé, le support roi est la télévision, une place qu'il se partage avec la presse magazine, la part de la radio est plus importante qu'en Allemagne, celle de la presse quotidienne étant négligeable.

La répartition des recettes entre les supports reflète l'importance respective des différents médias dans nos deux pays. Elle est elle-même le reflet du clivage nord-sud qui traverse l'Europe et qui fait des pays du nord ceux de l'écrit, des pays du sud, ceux du son et de l'image.

Malgré la très forte dynamique de l'audiovisuel depuis la libéralisation du secteur en Europe au milieu des années 1980, le média de référence allemand est et reste la presse quotidienne, avec plus de 350 exemplaires pour 1000 habitants (moitié moins en France). Bien que quelques grands titres comme la Frankfurter Allgemeine Zeitung soient diffusés sur l'ensemble du territoire, la presse est avant tout un média régional et local. Il en va de même de la radio, diffusée en bande FM depuis 1949 - à l'inverse de la France où, malgré la multiplication des antennes et décrochages (on y dénombre plus de 1800 radios contre 
seulement quelque 250 outre-Rhin), la radio reste un média national, diffusé en ondes logues depuis les origines (et depuis les années 1980 également en FM).

En Allemagne, bien que le service public propose entre autres 7 chaînes généralistes régionales, la télévision est le seul média 'national'. Surtout depuis l'ouverture du marché voici vingt ans et la multiplication des supports (câble et satellite) qui fait que, aujourd'hui, l'offre TV standard et gratuite comprend près d'une quarantaine de chaînes moitié publiques, moitié privées, alors qu'en France, malgré les balbutiements de la TNT, cette offre n'est toujours que de 6 chaînes. La structure de la réception diffère également : câble et satellite constituent outre-Rhin le mode standard, le hertzien terrestre étant résiduel - à l'opposé de la France où câble et satellite sont les vecteurs d'une offre complémentaire payante. De ce simple fait aussi, le poids des opérateurs de réseaux diffère.

Le marché allemand se caractérise donc par une offre pléthorique de médias classiques en comparaison d'une relative pénurie en France. Et, du point de vue du consommateur, par une grande diversité des sources d'information et de divertissement sur l'ensemble du territoire, par contraste avec la 'fracture médiatique' entre Paris et Province qui divise la France. Enfin, si la télévision est, dans nos deux pays pareillement, le média structurant pour l'individu comme pour la société, le système de référence proposé n'est pas le même : la majorité des Français regarde le 20 heures de TF1, celle des Allemands, le JT Tagesschau de la «Une » publique (ARD) dont la stricte déontologie est un héritage de la BBC. Car en droit comme de fait, le service public est outre-Rhin au fondement même de l'organisation de l'audiovisuel.

Dès lors, la 'révolution' du numérique n'a pas le même impact dans nos deux pays : Internet offre en Allemagne des opportunités complémentaires d'information et de divertissement, alors qu'en France, le Web tend souvent à suppléer une offre traditionnelle insatisfaisante et propose des canaux d'expression à une société civile tenue à distance des 'grands' médias.

\section{Des dualismes public/privé de l'audiovisuel eux aussi très différents}

Si dans nos deux pays, le segment de la presse a toujours relevé du marché, le segment de l'audiovisuel n'est devenu un marché, quoique particulier, que depuis sa libéralisation. Or le terme de « libéralisation » ne recouvre pas les mêmes réalités.

En France, il a deux acceptions : d'abord la levée du monopole d'Etat par la désétatisation de l'audiovisuel public avec, pour concrétiser sa nouvelle indépendance par rapport à l'exécutif, l'instauration d'une autorité indépendante (1982-86) ; ensuite, la privatisation d'une chaîne publique (TF1) en 1987. Depuis, en droit, le paysage audiovisuel français est un marché. Mais l'Etat a conservé une triple fonction de propriétaire du capital du service public, de législateur pour le cadre réglementaire dans lequel évolue l'audiovisuel, et enfin de régulateur direct pour les missions de service public, indirect pour celles du secteur privé et exercées par le CSA. Depuis, le dualisme public/privé porte essentiellement sur les statuts découlant de la propriété du capital comme des règles afférentes, à l'instar de ce qui prévaut sur le marché des télécommunications où coexistent opérateur historique et nouveaux entrants.

En Allemagne, où il n'y avait pas de monopole en droit, la libéralisation a été simplement synonyme d'ouverture à de nouveaux acteurs, issus du monde des médias, et à qui s'offrait là l'opportunité d'une diversification.. Jusqu'à aujourd' hui, aucun capital étranger 
aux médias, sinon d'origine financière, n'est présent dans l'audiovisuel, en vertu du 'tabou Hugenberg' (du nom de cet industriel et homme politique qui avait constitué un empire médiatique qu'il avait mis à disposition de Hitler). Cet interdit tacite, mais non moins puissant, ne simplifie pas l'approche allemande actuelle des phénomènes de convergence et de concentration à l'ère du numérique.

Or dans le pays de l'ordo-libéralisme, le nouveau dualisme public/privé devait être organisé. C'est le Tribunal constitutionnel fédéral qui, dans un arrêt rendu en 1986 et qui a valeur de grande Charte de l'audiovisuel, instaure "l'ordre dual de la radiodiffusion » (duale Rundfunkordnung). L'audiovisuel est ouvert au privé, à la condition que soient garantis le maintien et le développement du secteur public pour qu'il puisse continuer d'assurer sa mission, à savoir satisfaire l'ensemble des besoins de la communauté des citoyens (Grundversorgung). Comme le public assure ainsi une sorte de 'service universel', les obligations du privé peuvent se réduire à un standard minimum (Grundstandard).

Pour le dire autrement : l'existence d'un service public fort, financé par la collectivité, est la condition sine qua non pour la constitution de l'audiovisuel en marché. Mais cette définition se défend-elle encore face aux règles du marché communautaire ? Tient-elle encore à l'ère du numérique ? Aujourd'hui, certains commencent à revendiquer un 'Internet public'...

Troisième différence fondamentale avec le dualisme français : le système de régulation est l'émanation de la société civile organisée, non de l'Etat (exécutif ou administration), et réglementé par la loi. La Loi fondamentale (art. 5) interdit en effet toute implication de l'Etat dans les médias, quels qu'ils soient. C'est donc la collectivité qui assure, via ses représentants, le contrôle de l'audiovisuel, organisé de manière à garantir la pluralité des opinions jusqu'au sein même des instances afférentes.

\section{Fédéralisme/Etat centralisé}

Ce contrôle est assuré en interne, par un conseil de surveillance, dans chaque établissement de droit public (il y en a 11 au total); en externe pour le secteur privé, via une instance de régulation (Landesmedienanstalt). Comme les médias relèvent de la souveraineté des Länder en vertu de la répartition des compétences législatives au sein de la République fédérale, on compte 15 Landesmedienanstalten (Berlin et le Brandebourg ont un autorité commune), exclusivement compétentes sur le territoire de leur Land (ou Länder).

Si cette dualité de l'ancrage de la régulation n'est pas sans rappeler la situation française avec la Direction du développement des médias pour le secteur public, le CSA pour le privé, elle en diffère sur deux points au moins : l'entière autonomie statutaire et budgétaire des établissements de radiodiffusion public (et donc de leurs instances) comme des Landesmedienanstalten, de même que par le caractère de quasi juridiction dévolu à ces dernières. S'y ajoute un autre aspect, propre au fédéralisme allemand: le polycentrisme des décisions qui permet la coexistence et la mise en concurrence des diverses approches de gouvernance.

Ce polycentrisme implique que les questions d'intérêt commun soient réglées par voie contractuelle. Au niveau de la régulation, le processus de prise de décision présente une double articulation : les établissements publics, au statut de collectivité publique, agissent en pleine souveraineté à leur niveau ; les Landesmedienanstalten ont institué des plateformes communes comme la Conférence permanente de leurs directeurs (KDLM) par 
exemple. Au niveau de la réglementation, les Länder légifèrent sur les points d'intérêt commun par voie de traité, concluant des «Contrats d'Etat » (Staatsvertrag) qui, une fois ratifiés par les 16 parlements des Länder, ont valeur de loi sur l'ensemble du territoire. Le texte fondateur de l'ordre dual est ainsi le «Contrat d'Etat sur la radiodiffusion » (Rundfunkstaatsvertrag) de 1986, adopté conformément à l'arrêt des Juges constitutionnels et entré en vigueur en 1987 (il a été plusieurs fois amendé depuis). C'est par Contrat d'Etat également qu'est fixé par exemple, tous les 4 ans, le montant de la redevance pour l'audiovisuel public.

Or la souveraineté des Länder en matière de culture et de médias ne s'applique qu'aux contenus. La Fédération (Bund) est en effet compétente pour les questions relatives au marché : ainsi le droit de la concurrence dont le gardien suprême est l'Office fédéral des Cartels (Bundeskartellamt), et le droit sectoriel des télécommunications, un secteur dont les activités sont soumises à une autorité spécifique, en charge de la régulation des industries de réseaux, l'Agence fédérale des réseaux (Bundesnetzagentur).

En un mot: si les contenus relèvent des Länder, les 'tuyaux' relèvent du Bund. Cet éclatement des compétences entre les échelons territoriaux ne facilite évidemment pas l'approche allemande des phénomènes de convergence... La France centralisée peut les aborder d'autant plus facilement, malgré l'existence d'instances de régulation distinctes (CSA, ARCEP...), que médias ou télécommunications ont en outre ceci de commun que ces secteurs sont soumis tous deux au régime des concessions publiques.

\section{Des sources de droit divergentes}

Reste le point le plus épineux à l'ère du numérique : la difficulté à rendre compatibles droit commun et droit constitutionnel. Car si les télécommunications et les activités de réseaux relèvent largement du droit commun dans les deux pays, ce n'est pas le cas de l'audiovisuel : il relève foncièrement du droit constitutionnel. Si la culture juridique française permet néanmoins, bien que difficilement, de trouver des passerelles pour prendre en compte la convergence, la culture allemande l'interdit d'autant plus que se surajoute au conflit des sources du droit un conflit de compétences entre Bund et Länder.

Le Bundeskartellamt veille sur la concurrence en application de la loi fédérale contre les entraves à la concurrence (Gesetz, gegen Wettbewerbsbeschränkungen, GWB). Le Tribunal constitutionnel fédéral (Bundesverfassungsgericht) veille sur les droits fondamentaux. Ses arrêts ont force de loi et sont immédiatement applicables. Or c'est lui, via une abondante jurisprudence, qui a déterminé l'organisation de l'audiovisuel allemand de manière à ce qu'il puisse assumer sa fonction de «facteur éminent dans le processus de formation de l'opinion » (arrêt de 1961), une fonction intrinsèque à tous les médias mais qui a été foncièrement ancrée dans les missions de l'audiovisuel public depuis l'arrêt de 1986 sur «l'ordre dual ».

Autrement dit : la prise en considération, sous l'angle de la régulation, de la convergence des contenus et tuyaux qui s'esquisse dans les médias - qui sont un marché de facto, mais seulement en partie considéré ainsi par le droit, se heurte aussi aux valeurs et principes de la Constitution. L'ère du numérique ne défie pas seulement l'entendement, pas seulement le droit. Il défie aussi la philosophie du droit en plaçant au cœur des enjeux le destin d'un audiovisuel (public) qui était jusqu'ici le pivot de la conception des médias de masse et du lien social à la fois. 


\section{Discussion}

Bénédicte de Peretti : La pluralité des supports décuple l'offre. Télévision mobile, podcast et autres nouveaux services modifient le mode de consommation du téléspectateur : alors qu'il était jusqu'ici passif face à une offre sur laquelle il n'avait aucune maîtrise, il peut désormais composer lui-même cette offre. Cette convergence des services remet en question la définition établie de la radiodiffusion ...

Francis Balle : ...le mot convergence prête à équivoque parce que deux images brouillent notre perception de ce qu'est la convergence aujourd'hui. La première image nous vient du début des années 1980 lorsqu'on avait découvert qu'à l'aide d'un même fil, en l'occurrence la fibre optique, on pouvait aussi bien acheminer de la voix que des images de télévision. Par conséquent on s'était imaginé, un peu trop vite, que les mêmes tuyaux allaient pouvoir acheminer toutes sortes de messages qui autrefois empruntaient des réseaux dédiés les uns à la radiodiffusion, les autres aux télécommunications.

La deuxième image vient du fait que nous nous soyons imaginés que nous allions avoir tous un seul écran pour faire ce que différents écrans faisaient auparavant. Or nous constatons aujourd'hui deux choses : tout d'abord, on a vu se multiplier les réseaux, même si chacun de ces réseaux peut faire des choses différentes ; puis on a vu aussi se multiplier les terminaux, et nous avons aujourd'hui quatre catégories d'écrans : un très grand pour le cinéma, un moyen pour la télévision, un plus petit pour l'ordinateur et enfin l'écran du téléphone portable. Techniquement, chacun de ces terminaux peut tout faire, ce qui ne veut pas dire que nous n'ayons pas à l'avenir une démultiplication des services et qu'il y aura plutôt une affinité entre certains écrans et certaines catégories de services : il est clair que si on peut recevoir un film de télévision sur un écran de téléphone, ce n'est pas le meilleur moyen de regarder un film.

Le mot convergence est donc équivoque puisqu'on assiste en réalité plutôt à une divergence, à une multiplication des réseaux et une démultiplication des terminaux. Il faut dissiper une autre équivoque encore : il y a quelques années, on pensait que seule l'alliance entre les contenus et les contenants pouvait faire naître de nouveaux services et que les clefs de l'avenir sont détenues pas ceux qui maîtrisaient à la fois les contenants et les contenus. Il suffisait par conséquent d'être présent sur ces deux segments pour faire naître des programmes ou des services prometteurs, pensait-on alors.

L'emploi indifférencié du terme de convergence me semble donc aujourd'hui d'autant plus risqué que subsiste malgré tout la distinction philosophique, juridique et politique entre d'une part la communication publique (le point-multipoints) et, d'autre part, la communication privée (le point à point). La communication publique est forcément limitée : on ne peut pas tout dire lorsqu'on s'adresse à un très large public et, dans n'importe quel régime, il existe des limitations à la liberté d'expression lorsque celle-ci emprunte la communication publique. En revanche, la communication privée est parfaitement libre et obéit au secret de la correspondance privée. La difficulté réside dans le fait qu'aujourd'hui, depuis la diffusion du Web, on voit apparaître une communication 
intermédiaire, d'un troisième type : chacun peut constituer une communauté avec des médias, des vecteurs, dont on n'avait pas idée il y a seulement quelques années.

Il est vrai que nous n'avons pas de mot en français pour traduire broadcasting. On parle de broadcasting, de narrowcasting, de podcasting, mais en réalité, ce sont trois modalités de l'échange qui doivent obéir à des règles différentes, qui n'empruntent pas les mêmes tuyaux et qui n'apportent pas les mêmes services. On ne peut évidemment pas comparer un service de diffusion large comme la radiodiffusion sonore ou la télévision avec la communication privée ou avec ces nouveaux services qui relèvent tantôt de la communication publique, tantôt de la communication privée selon que je projette sur mon écran d'ordinateur la vitrine d'un site pour savoir ce que je vais acheter ou selon que je vais procéder à un acte d'achat, c'est-à-dire instaurer une communication privée, avec quelqu'un qui va me fournir un service, à qui je vais donner mon numéro de carte bancaire et où je demande à être sécurisé et à bénéficier de la même protection que lorsque j'échange une lettre avec un correspondant.

\section{La rareté des supports exige-t-elle une régulation spécifique?}

Bénédicte de Peretti : Si les frontières entre ces différentes modalités sont de plus en plus floues, c'est dû aussi à la multiplication et à la diversification des infrastructures de diffusion. Or c'est leur rareté qui légitimait jusqu'ici le statut particulier réservé à l'audiovisuel. Celui-ci peut-il encore être maintenu ?

Bernd Holznagel : En Allemagne, le Tribunal constitutionnel fédéral n'a jamais fondé le rôle particulier de la l'audiovisuel sur la rareté des fréquences. Il l'a justifié par l'influence qu'il peut avoir sur la formation de l'opinion, sur la démocratie. C'est cela qui, aux yeux des juges suprêmes, nécessite également une régulation sectorielle spécifique ; l'association de l'image et du son a en effet une force suggestive particulière.

Il ne s'agit pas là d'arguties juridiques. En politique comme en droit sont seuls considérés les services médiatiques qui contribuent au processus de formation de l'opinion dans la démocratie. Il s'agit dès lors d'identifier ceux-ci au sein d'une offre plus vaste. Peu importe en définitive qu'on les nomme point à point ou point-multipoints ou qu'on leur applique la définition des juges de Karlsruhe. Pour ma part, je n'ai aucun doute que, parmi ces services, il en existe certains qui ont un pouvoir d'influence sur l'opinion, et je ne souhaite pas que quelques magnats isolés puissent grâce à ceux-ci former l'opinion selon leurs désirs.

Reste la question de savoir s’il convient également de soumettre à la régulation sectorielle de l'audiovisuel les services liés à Internet ; mais c'est là un autre sujet. Il n'y a aucun doute de principe sur la nécessité d'une régulation spécifique de l'audiovisuel.

B. de Peretti : Pourtant, le comportement de nos enfants, par exemple, révèle que les nouveaux consommateurs ne regardent pas toujours les programmes qui leur sont destinés, mais composent au contraire leur propre bouquet. Que reste-t-il dès lors du pouvoir d'influence sur l'opinion prêté à l'offre classique ? Cette évolution ne plaide-telle pas plutôt pour une modification de la régulation de l'audiovisuel ?

Uwe Kammann : Je ne partage pas entièrement l'approche de Bernd Holznagel. Je pense au contraire qu'il nous faut des dispositions particulières à l'audiovisuel étant donné que la rareté des supports de distribution, de même que le volume élevé des 
investissements financiers nécessaires augmentent le risque d'une prise d'influence sur l'opinion par l'opérateur. C'est ce qui distingue justement l'audiovisuel de la presse écrite, où on estime généralement que la liberté ou le pluralisme d'opinions naissent d'une offre plurielle et diversifiée entretenue par la concurrence sur le marché de la presse. Le pouvoir d'influence d'un seul support se trouve par là même réduit.

B. Holznagel : C'était là l'analyse faite par le Tribunal constitutionnel fédéral dans son premier jugement sur la radiodiffusion (1961). Mais dès les $2^{\mathrm{e}}$ et $3^{\mathrm{e}}$ arrêts (1971 et 1981), la cour suprême a abandonné cette argumentation...

U. Kammann : ... cela ne change rien à la question de fond qui est de savoir si les prémisses adoptées aux débuts de l'audiovisuel peuvent être maintenues dans le contexte actuel des mutations. Jadis, peu d'acteurs avaient les moyens techniques et financiers pour s'adresser à la masse, ce qui réduisait l'éventail des opinions exprimées et comportait un risque de manipulation. Aujourd'hui, au contraire, on observe la montée en puissance d'une communication individualisée.

Il y a vingt ans encore, nous nous plaisions à citer Bertolt Brecht qui plaidait pour une «démocratisation» de la radio et rêvait d'une situation où, au lieu d'avoir un émetteur s'adressant à la masse, chaque consommateur pourrait être lui aussi un émetteur. C'est, du moins potentiellement, le cas aujourd'hui, avec la multiplication des communautés d'internautes, où la communication individuelle se transforme en communication de masse bien que, techniquement et juridiquement parlant, elle relève du point-à-point.

Je partage le constat de Francis Balle sur cette situation paradoxale qu'on peut observer actuellement, où communication de masse et communication individuelle tendent à se confondre, et où coexistent toutes les formes intermédiaires possibles et imaginables. C'est dans cette dilution des frontières d'ensembles autrefois bien distincts que réside la convergence : à tout moment, en tout lieu et grâce aux supports les plus divers, la réception de contenus de toute nature est désormais possible, tout comme son émission.

La notion d'audiovisuel a dès lors perdu de sa consistance. Naguère, le Tribunal constitutionnel fédéral définissait la radiodiffusion comme l'organisation et la présentation, par le son, la parole et l'image, de contenus destinés à la collectivité, au moyen d'ondes électromagnétiques. Certes, le mode de distribution n'a pas changé : même si les applications se sont diversifiées, elles mettent toujours en œuvre les ondes électromagnétiques. Mais que peut-on considérer encore comme destiné à la collectivité, à la masse ? Si je veux distribuer un contenu au client final, je peux aussi le faire par abonnement, donc à un cercle restreint d'utilisateurs, ou bien à l'acte, et dans ce cas, mon client unique achète mon émission comme s'il achetait un journal au kiosque. Cela amène à repenser la notion d'audiovisuel et pose un défi à l'approche de sa régulation.

F. Balle : Ce que je crois, c'est qu'aucun média n'est jamais évincé par un autre, le nouveau n'évince pas l'ancien et on n'assiste pas du tout aujourd'hui à l'éviction de la radiodiffusion au sens classique point-multipoints, mais à la juxtaposition de formes différentes de communication. Il n'existait en 1960 que deux formes de communication parfaitement canoniques : le point à point (c'est le secret de la correspondance privée) et le point-multipoints, cette forme de communication devant être soumise à un autre régime juridique avec des protections particulières afin d'éviter que la libre expression de certains porte atteinte à d'autres libertés tout aussi importantes. 
Il est vrai qu'on voit se juxtaposer des formes intermédiaires de communication, mais lorsque j'utilise mon ordinateur pour procéder à un acte d'achat, lorsque je me mets devant une vitrine, dans un premier temps, je suis dans le cadre d'une communication publique qui obéit aux lois relatives à ce type de communication. Puis, si je veux procéder à un acte d'achat, je suis alors dans le cadre d'une communication privée où je suis en droit de réclamer la protection que j'ai lorsque j'échange une lettre avec quelqu'un. C'est en cela que le mot convergence nous induit en erreur et que «Bruxelles » a commis quelques petites erreurs en voulant aligner toute la communication sur les télécommunications, comme si la radiodiffusion (le broadcasting) allait se noyer dans les télécommunications. Or ce n'est pas le cas, puisque la communication publique ne peut pas ne pas obéir à des lois particulières qui sont celles des libertés publiques.

\section{Concentrer l'effort de régulation sur les nouveaux gate keepers ?}

B. de Peretti : Par-delà ces divergences, il semble pourtant y avoir un consensus quant à la nécessité de moderniser et de libéraliser la réglementation de l'audiovisuel ?

B. Holznagel : Je voudrais revenir sur l'analyse d'Uwe Kammann, que je ne partage pas. L'Allemagne a abandonné la notion de radiodiffusion il y a une dizaine d'années. $\mathrm{Si}$ on quitte le terrain juridique, on peut considérer qu'il y a trois catégories de services médias.

La première est celle de l'audiovisuel classique (broadcasting), et nous lui appliquons les instruments établis, c'est-à-dire le droit des concentrations, la réglementation des quotas, etc. La seconde catégorie est celle de la presse électronique. Il s'agit de journaux diffusés en ligne et qui pourront l'être également via la télévision. Cette catégorie porte le nom de «services médiatiques »(Mediendienste), et elle est soumise à la même réglementation que la presse écrite.

La troisième catégorie est celle des «télémedias »(Telemedien). Elle correspond à ce qu'a décrit Francis Balle et se concentre sur l'acte de transaction : j'achète un bien et le paie en ligne ou en effectuant un virement, par exemple. Dans ce cas, évidemment, aucune réglementation spécifique aux médias ne s'applique. Celle-ci ne s'applique qu'aux deux autres catégories : sous sa forme classique à la première, sous une forme allégée à la seconde.

En droit constitutionnel allemand, la deuxième catégorie relève certes de l'audiovisuel. Mais il y a une dizaine d'années, nous avons, je le rappelle, sorti du périmètre de l'audiovisuel une série de services et allégé la régulation pour ceux-ci bien que, en droit constitutionnel, ils relèvent toujours du concept de l'audiovisuel. La question de savoir ce qui relève de l'audiovisuel ou non ne se pose donc plus en ces termes-là. Elle a en outre trouvé une réponse voici dix ans avec la signature, par les Länder, du Contrat d'Etat sur les services médiatiques (Mediendienstestaatsvertrag). Nous avons donc défini dans ce texte une série de services qui ne portent pas le nom d'audiovisuel et qui sont soumis à une autre réglementation, distincte de l'approche classique étant donné le risque moindre pour le pluralisme des opinions. Nous avons depuis, en Allemagne, un système de régulation très différencié.

B. de Peretti : Mais ces services sont en pleine évolution. Et n'y a-t-il pas un risque, à vouloir maintenir une régulation particulière pour ces médias classiques, que ceux-ci voient se réduire à moyen terme leur importance ou leurs parts de marché ? 
B. Holznagel : Il n'est effectivement pas à exclure que le poids de l'audiovisuel, des services point à multipoints, se réduise au détriment du podcasting, comme nous appelons ces services point-à-point. Sur 100000 services, il pourrait ne plus y avoir à terme que cinq soumis à une régulation stricte, le reste relevant de la régulation assouplie. Mais ce n'est là qu'une approche purement économique. Car ces 'nouveaux' services que consomment nos enfants ont un rôle secondaire en ce qui concerne le pluralisme des opinions. Ce qui compte, en réalité, c'est que nous continuons à consommer les services classiques de télévision.

B. de Peretti : La réalité est pourtant autre : de plus en plus de citoyens s'informent sur Internet et non plus par la télévision. Ne peut-on pas en conclure que la télévision, en tant que média d'information, est en perte de vitesse ?

B. Holznagel : Oui, dans la mesure où des moteurs de recherche de type Google proposent un accès à l'information. Et il est vrai aussi que les enfants ne tombent pas par hasard sur les contenus qu'ils cherchent ; au contraire, ils se laissent guider par des moteurs de recherche et la confiance en une marque. Et ces outils de navigation qui nous guident dans la masse de l'information ont un rôle croissant de gate keepers ; ce sont eux qui, à l'avenir, auront un rôle décisif dans la formation de l'opinion.

Les économistes, experts des médias et professionnels réunis au sein du think tank sur la société de l'information, le 'Münchner Kreis', par exemple, estiment à l'unanimité que ce ne sont ni les opérateurs de l'audiovisuel ni les opérateurs des télécommunications qui seront les acteurs clé de demain, mais les géants d'Internet, autrement dit, les Google et autres. Je ne partage pas leur avis, mais les économistes notamment ont esquissé le scénario suivant : les géants du Web investissent leurs bénéfices dans le rachat de groupes de médias classiques, ce qui devrait se traduire par une chute des recettes publicitaires dans le secteur privé de l'audiovisuel et pourrait ainsi le condamner à terme. Il ne resterait plus dès lors que deux segments : Internet et l'audiovisuel public...

B. de Peretti : ... mais Internet n'est pas régulé...

F. Balle : ... c'est vrai, mais dans ce cas, on a affaire à une compétition entre entreprises qui tentent de conquérir un marché. Aussi longtemps que Google ne jouit pas d'une position hégémonique, nous sommes dans une situation de marché où le droit de la concurrence s'applique pleinement. Par conséquent, à mesure qu'on entre dans une ère de moindre rareté, on voit de plus en plus le droit de la concurrence prendre le pas sur tous les autres droits sectoriels.

Quand on a affaire à dix radios dans un même lieu, les règles concernant le pluralisme ont moins d'intérêt que lorsqu'on a affaire à une seule radio. Quand on est dans une situation de monopole comme nous l'étions en France jusqu'au tout début des années 1980, il était important de régler le pluralisme par une autorité aussi indépendante que possible. C'était la justification donnée - et qui primait sur la rareté - pour créer la première autorité qui a donné naissance ensuite à d'autres autorités dont le CSA est l'héritier. A mesure qu'on s'éloigne de la rareté et qu'on va vers des situations de relative ou de grande abondance, les règles concernant le pluralisme perdent de leur intérêt, et le droit de la concurrence occupe le terrain laissé vacant par les réglementations concernant le pluralisme.

U. Kammann : Bernd Holznagel fonde sa réflexion sur la situation actuelle qui se caractérise par une distinction nette entre les services et, partant, une claire répartition 
des compétences entre le Bund et les Länder, ces derniers étant compétents pour l'audiovisuel, et la Fédération, pour les «télémédias ». Seulement, je pense que le concept classique d'audiovisuel ne résistera pas longtemps à l'évolution des médias, puisqu'il était centré sur la vision d'un opérateur offrant un ensemble linéaire, construit, de services au moyen d'un support ou d'une plateforme unique ; en outre, il part d'un nombre d'opérateurs et de plateformes limité.

Or c'est cette définition du programme comme de la fonction d'opérateur qui tend à se diluer. La BBC, par exemple, se voit à l'avenir comme un offreur de contenus audiovisuels mettant à disposition des émissions à l'unité que le consommateur pourra choisir en ligne en consultant un catalogue virtuel. Mais dès lors que je rends un film ou une émission accessibles en ligne, que je sois éditeur ou intermédiaire, je suis un opérateur de l'audiovisuel à part entière, puisque c'est moi qui détiens le contenu en question.

En ce qui concerne l'offre de contenus, nous réglementons et contrôlons jusqu'ici les opérateurs sous l'aspect de leur organisation. Cela n'aura plus guère de sens dans cinq ou dix ans, puisque la catégorie des offreurs de programmes se diversifie : elle inclut déjà les opérateurs d'infrastructures, de même que ceux qui composent des bouquets de programmes. De plus, la multiplication des alliances entre ces diverses catégories d'acteurs rendra inopérante notre approche établie de régulation. En Allemagne, cela remet également en question cette double régulation qui repose sur le partage des compétences entre Bund et Länder.

Autrement dit : il ne sera plus possible de définir l'audiovisuel - la communication point-à-multipoints - par les risques sur le pluralisme des opinions comme nous le faisions jusqu'ici. Il nous faudra d'autres critères : dans un marché en structuration où pourraient se constituer de nouveaux monopoles, la régulation devra plus nettement être centrée sur le client ou le consommateur, et non plus sur l'opérateur.

Le pivot de l'organisation future des médias : l'approche du service public de l'audiovisuel

B. de Peretti : Dans cette situation, qu'adviendra-t-il du financement par la redevance ? Devrai-je payer une redevance dès lors que je compose mon propre programme TV sur mon téléphone portable ? Certains Etats de l'UE comme le Portugal ou les Pays-Bas ont remplacé la redevance par une taxe prélevée en fonction du revenu des ménages...

B. Holznagel : Il n'y a pas lieu de supprimer la redevance, indépendamment du fait qu'elle contribue à financer les instances de régulation de l'audiovisuel des Länder (à hauteur de $2 \%$ du total). La question est de savoir qui, des trois types d'acteurs, sera en position hégémonique : les opérateurs de l'audiovisuel, les 'Googles' ou les opérateurs de télécommunications ? La réponse dépend du scénario qui s'esquissera.

Si les 'Googles' s'imposent, cela signifie la fin de l'audiovisuel privé, puisque tous les deux se financent par la publicité. Et comme ce scénario ne laissera pas indifférent le monde politique, opposé à l'américanisation des médias, le gagnant évident sera dans ce cas le service public de l'audiovisuel. Nous aboutirions dans ce cas à une double 'remonopolisation": celle des opérateurs de télécommunications et celle des opérateurs publics de l'audiovisuel, déjà largement intégrés verticalement. Le montant de la redevance sera alors encore plus élevé qu'aujourd'hui.

Second scénario : si les opérateurs de télécommunications parviennent à s'imposer parce qu'ils maîtrisent l'ensemble de la chaîne de création de valeur, le service public 
sera affaibli. Etant donné que le modèle économique des opérateurs de télécommunications repose sur une offre cryptée, payante, il y aura moins d'espace pour une offre gratuite obéissant à une mission d'intérêt général.

Enfin, troisième scénario : celui qu'évoquait Uwe Kammann, à savoir l'individualisation totale des médias. S'il se réalise, il conviendra d'abandonner le système actuel de régulation reposant sur la garantie du pluralisme; les Länder se réuniront pour abroger leurs lois sur l'audiovisuel, de même que les Contrats d'Etat qu'ils ont signés conjointement et qui ont force de loi sur l'ensemble du territoire allemand. Mais pour cela, il faut que la situation ait réellement changé. Le législateur n'agit pas au vu de simples scénarii.

F. Balle : Il est tout à fait normal et compréhensible que les grandes radios ne laissent pas le marché du podcasting leur échapper. Elles ne veulent pas voir arriver des concurrents, elles veulent garder ce marché pour elles-mêmes, en empêchant d'autres de le conquérir. Mais cela ne veut pas dire pour autant qu'elles soient pessimistes sur leur propre avenir car il y a de l'avenir pour les radios généralistes comme il y a de l'avenir aussi pour les télévisions et les journaux généralistes. Il ne faut pas sous-estimer la paresse des gens. Ce n'est pas parce que vous avez la possibilité de choisir vos différents contenus que vous n'avez pas envie de temps en temps, quand vous rentrez chez vous à 20 heures, de regarder malgré tout le journal télévisé sur une grande chaîne généraliste pour faire le tour de l'actualité en 20 minutes (c'est le nom précisément d'un journal qui est gratuit aujourd'hui). Par conséquent, les généralistes ont autant un avenir que la vente des contenus 'à l'acte'.

On assiste aujourd'hui à une multiplication non seulement des modes de distribution, des formes de communication, mais aussi des modèles économiques, car n'oublions pas que la télévision a d'abord été gratuite, qu'elle a ensuite été payante au forfait et qu'aujourd'hui elle est payante à l'acte. Mais les trois modèle existent en parallèle : on a toujours la télévision gratuite (payée par la redevance ou par les annonceurs), on a la télévision au forfait $(\mathrm{Canal}+)$ et on a la télévision à l'acte.

La presse prend exactement le chemin inverse : la presse a d'abord été très chère, puis un peu moins chère quand elle a été payée en partie par les annonceurs, et elle est maintenant gratuite. Quand les journaux payants ont vu la presse gratuite arriver, ils ont affirmé qu'il ne s'agissait pas de journaux. Le premier journal en termes de diffusion en France est aujourd'hui un gratuit qui est distribué dans les transports en communs dans les plus grandes villes de France. Il est gratuit à $100 \%$ comme l'était la télévision. On ne s'indignait pas que la télévision soit gratuite il y a quelques années, pas plus qu'il ne faut s'indigner qu'aujourd'hui la presse, de temps en temps, puisse être gratuite.

L'autre grand problème que vous avez soulevé tout à l'heure est de savoir où placer le curseur entre ce qui doit être soustrait à la logique marchande et ce qui doit être laissé au marché. Faut-il conserver un espace où c'est la logique de service public qui joue et non pas la logique du marché ?

J'aimerais rappeler que les Américains sont partis avec une logique inverse dans la télévision et ont commencé par appliquer le régime de la concurrence privée en 1967. Des hommes du Congrès et de l'université sont allés voir la fondation Carnegie en lui demandant un peu d'argent pour voir comment ils pourraient soustraire une partie de la télévision à la logique marchande. Ils étaient, les uns comme les autres, catastrophés devant ce que devenait la télévision. C'était en 1967 et cela a donné naissance au réseau 
public américain, dont certes on peut dire qu'il ne touche qu'une minorité de l'audience américaine. Cela dit, il faut quand même rappeler que le réseau PBS a joué un rôle considérable pour les autres télévisions et que tout ce qui s'est fait de nouveau en télévision dans le domaine de l'information à travers les journaux de PBS à $18 \mathrm{~h}$ et dans le domaine de l'éducation au travers des programmes pour enfants comme Sesam Street a été fait dans le cadre de la télévision publique.

Le drame est que, en Europe, nous avons un peu mélangé les genres et que nos télévisions publiques ressemblent trop souvent, et de plus en plus, à des télévisions privées, de telle sorte que, comme les Japonais il y a quelques années, nous allons peut-être nous demander pourquoi payer une redevance alors qu'au fond nos chaînes dites publiques ressemblent de plus en plus aux chaînes privées. Une grève a été organisée par les Japonais récemment pour ne plus payer la redevance à la NHK.

Nous n'en sommes pas là, mais il faut préserver un secteur non marchand pour développer des préférences collectives ou atteindre des objectifs qui aujourd'hui ne sont pas satisfaits par la logique marchande des télévisions privées.

Définir des services audiovisuels d'intérêt général ?

U. Kammann : Je ne partage pas l'analyse de Francis Balle. Le service public diffère foncièrement du privé. Peut-être plus en Allemagne qu'en France, il est vrai. Mais dans ce cas, il faut se demander si le CSA dispose réellement des moyens adaptés à sa mission. Dès lors qu'un système audiovisuel est doté d'une mission d'intérêt général pour la collectivité, la société a le devoir de veiller à ce qu'il remplisse cette mission dans le respect du pluralisme, de la liberté et de l'indépendance. Malgré une tendance au nivellement qu'on peut observer dans certaines émissions et qui reflète une certaine convergence des contenus dans un environnement concurrentiel, le service public allemand remplit dans l'ensemble ses missions. Il est tout simplement tenu de le faire pour garantir l'existence d'un espace de communication libéré des contraintes économiques et accessible à tous les citoyens, qu'ils profitent de cette offre ou non.

Un service d'intérêt général est aussi indispensable en matière de communication qu'en matière de santé ou d'éducation, des domaines où il est considéré comme allant de soi que la société finance ces prestations par un impôt ou une redevance. J'estime que, dans le champ des médias, cette mission incombe en priorité à l'audiovisuel, puisque la presse est payante pour le client et que les autres services ne sont pas en mesure de garantir un éventail suffisamment libre et pluraliste d'informations.

Car les médias éduquent et cultivent, pas seulement en diffusant des émissions politiques ou des magazines d'information, mais aussi à travers la fiction. En donnant du sens à ma vie, ils me permettent de forger et de développer mon identité.

En comparaison, la question de savoir comment doit être prélevée la redevance ou si elle doit être remplacée par une taxe, comme en France, est accessoire. L'audiovisuel a une mission publique au financement de laquelle chacun doit contribuer à un coût accessible. Les Allemands ne mettent pas en doute la légitimité du service public de l'audiovisuel, et la majorité paie d'ailleurs volontiers la redevance, consciente de participer ainsi à une communication équitable.

B. de Peretti : Reste la question de la justification de la redevance dans un environnement désormais ouvert à la concurrence. Madame Reding exige plus de transparence 
et de rigueur en Allemagne, ce qui montre bien, malgré toutes les qualités des prestations du service public, qu'il y a un problème...

B. Holznagel : Il est vrai que, ces dernières années, l'audiovisuel public allemand n'a pas saisi la chance qui lui offre son autorégulation pour redéfinir sa mission de service public. Dieter Stolte, le président de la ZDF, s'était certes engagé en ce sens en 1999, mais il n'a pas été suivi par ses homologues des autres établissements de radiodiffusion publics. Je ne suis donc pas opposé par principe au fait que Bruxelles intervienne dans le domaine de la définition des missions du service public ni dans d'autres où la politique audiovisuelle allemande s'est un peu reposée sur ses lauriers.

Je me dois en effet, dans le cadre d'un système dual, de définir les rapports de concurrence qui existent entre le privé et le public. Or on n'a par exemple jamais clarifié les moyens et les domaines d'action des filiales du groupement public ARD sur le marché, ni les relations entre les établissements de droit public membres de ce groupement et leurs filiales commerciales de statut privé. Les ministres-présidents auraient dû réagir depuis longtemps et mettre en place les règles de transparence requises. Mais ils ne l'ont pas fait car les établissements ont trop de pouvoir dans leur Land respectif.

Or cette question reste un défi pour la régulation de l'ordre dual qui caractérise le marché audiovisuel allemand. Il ne s'agit nullement de remettre en question l'existence du service public, un point de vue que je partage avec Uwe Kammann. Ce qui est en jeu, c'est la création d'un cadre réglementaire approprié. Dans les années qui viennent, les débats sur ce dernier vont se multiplier. Par exemple en ce qui concerne l'utilisation souvent peu efficiente des fréquences par le service public ; on peut s'attendre à ce que le secteur des télécommunications exerce une certaine pression qui aboutira aux réajustements qui s'imposent.

\section{Une sorte de service universel pour préserver le lien social ?}

B. de Peretti : Un peu de prospective... Comment les chaînes traditionnelles peuventelles affronter les profondes mutations des médias?

U. Kammann : En définissant clairement les missions du service public. Comme les établissements publics n'ont pas été capables de le faire, il revient désormais au législateur de préciser les missions qui incombent au service public. Mais la loi n'en devra pas moins laisser aux chaînes une large autonomie en termes de programmation.

Sur un point, l'Allemagne ne partage pas l'approche de Bruxelles. Il s'agit du projet d'interdire aux chaînes publiques la diffusion de grandes émissions de divertissement ou la retransmission d'événements sportifs au double motif que l'intérêt général ne justifie en rien le financement de ce type d'offre par la redevance et que celui-ci mène à une distorsion de la concurrence au détriment des chaînes privées. Cela étant, une telle limitation de l'offre des chaînes publiques peut se défendre - mais à une condition seulement : que, parallèlement, le législateur considère que, dans certaines catégories de programmes, la demande est si bien servie par tous les prestataires que plus rien n'y justifie le régime d'exception d'un service public protégé par son statut et financé par la redevance.

Quant au secteur privé, il va changer radicalement. Les opérateurs propagent déjà de nouveaux modèles économiques car ils constatent que leurs recettes publicitaires reculent dans leurs activités audiovisuelles au profit des opérateurs de plateformes dont la 
part de marché publicitaire s'accroît. Le concept de télévision payante va ainsi se développer, et la relation de l'émetteur au récepteur sera plus individualisée. On paiera de plus en plus pour des contenus uniques, de sorte que les programmes de structure linéaire perdront en importance. Ce seront peut-être les producteurs ou les distributeurs qui transmettront les contenus et qui devront partager ce pouvoir avec les opérateurs de plateformes. Et ce sont eux qui détermineront ce qui sera proposé au client, dans quel éventail de prestations et à quel prix.

Une nouvelle structuration de la concurrence s'esquisse. Je ne pense pas qu'un monopole se mette en place aussi rapidement qu' on le dit ou que ce soit uniquement un 'Google' qui s'impose. Mais les plus exposées aux mutations sont assurément les chaînes privées. Il y aura une plus forte organisation en conglomérats mais aussi une plus forte fragmentation des opérateurs, puisque de nombreuses petites structures établiront des relations commerciales par l'intermédiaire d'offres spéciales.

On observera, comme c'est le cas des revues scientifiques, une fragmentation croissante de l'offre de même qu'une hausse des prix qui exclura une grande partie du public. L'Histoire, pour une fois, pourrait se répéter ... Celui qui avait de l'argent pouvait investir dans la culture, acheter des livres, des revues, s'informer aux sources les plus prestigieuses et perfectionner ses connaissances. A l'avenir, l'accès à l'information, à la culture et à la connaissance sera plus cher, ce qui contribuera à une fragmentation de la société plutôt qu'à son intégration. Il reviendra alors aux chaînes publiques de contrebalancer ces effets en étant une ressource accessible à tous, pour autant qu'ils soient en mesure de et disposés à le faire. Cela plaide pour garantir une sorte de 'service universel' afin de réduire les risques d'exclusion et de préserver le lien social.

F. Balle : Je pense moi aussi qu'il faut préserver un secteur non marchand pour les préférences collectives. Mais je crois pour ma part que l'avenir du système français dépendra de la clarification des objectifs que l'on saura donner le plus solennellement possible au secteur public. Pour l'instant, on ne peut pas dire que ces objectifs aient été clairement proclamés au point de justifier que de l'argent public aille vers la production de contenus que les stations ou chaînes privées ne seraient pas susceptibles de produire elles-mêmes.

\section{Reste à définir ces missions d'intérêt général et d'en contrôler l'application}

Or en ce qui concerne le service public, le CSA doit se contenter d'un rôle de 'gendarme' : il veille au respect de règles qui n'ont pas été édictées par lui. Le comble de l'ironie est que pour les opérateurs privés, il subordonne toujours l'autorisation de diffuser à la signature d'une convention où il fixe, en accord avec le bénéficiaire, un certain nombre d'obligations particulières. Le CSA détermine ainsi des 'obligations de service' pour les diffuseurs privés, alors que dans le cas des chaînes publiques, il ne fait que veiller au respect d'obligations qui ont été fixées dans le secret des bureaux du ministère de la Culture et de la Communication ou de la Direction de développement des Médias du Premier Ministre.

La Commission européenne joue un rôle décisif en estimant que quand il y a de l'argent public, il faut aussi qu'il y ait une justification du caractère public des contenus qui bénéficient de cet argent. Or jusqu'à récemment, la comptabilité des chaînes publiques ne distinguait pas clairement entre le financement des programmes qui étaient 
ceux susceptibles d'être diffusés par des chaînes privées et des programmes qui marquaient la spécificité des chaînes publiques, justifiant leur existence. Je souhaite moi aussi très ardemment la survie des chaînes publiques, à condition que leurs objectifs soient clairement définis.

Nous nous trouvons actuellement, en France, face à un dilemme : nos chaînes publiques sont extrêmement importantes en audience, en nombre de personnes mobilisées, en argent public prélevé, alors que les objectifs de fond ne sont pas clairs pour beaucoup de gens. Ces derniers ne sont malheureusement pas déterminés par une autorité indépendante comme le CSA, ce qui devrait être le rôle d'une autorité vraiment indépendante comme elle le montre avec les chaînes privées. Ainsi nous nous situons à la croisée des chemins. Il est vrai que nos chaînes publiques ont été un peu dénaturées, détournées des objectifs légitimes qui leur avaient été fixés par les fondateurs lorsque que la télévision était encore un monopole - ce qui Dieu merci n'est plus le cas depuis longtemps.

Jacques Rigaud (dans la salle) : Je voulais confirmer ce que vient de dire Francis Balle sur la dissolution du service public en ce qui concerne les chaînes de télévision. En qualité d'ancien président de la première radio commerciale française (RTL), je suis de l'avis qu'il existe une nette différence entre la radio et la télévision quant au respect de la notion du service public. Nous avons encore un groupe de radio - Radio France qui, bien qu'il diffuse des publicités, conserve un véritable esprit de service public quant au contenu des programmes, quant au pluralisme (qui est maintenant beaucoup mieux respecté dans les domaines de la radio et de la télévision par le pouvoir politique). Je crois qu'il faut insister sur cette différence, pour éviter qu'on ne croie qu'en France, on a complètement perdu l'esprit de service public.

Je serai encore plus sévère que Francis Balle en ce qui concerne la télévision et la déperdition complète de l'esprit de service public, notamment dans l'information, ce qui n'est pas vrai pour la radio. De plus, il n'y a en réalité personne, au niveau de l'Etat, qui ait une conception politique du rôle des médias. Le ministre de la Culture, quel qu'il soit, tient au moment de son arrivée des propos nobles sur la vocation de service public de la télévision, propos qui sont sans aucune conséquence et qui ne gênent personne, à commencer par les responsables de la télévision de service public. La Direction de développement des Médias, qui relève du Premier Ministre, est un service compétent, sérieux, bien organisé, mais qui n'a aucun pouvoir propre, le seul tuteur du service public de l'audiovisuel en France étant le Ministre des Finances, avec la hauteur de vue que l'on peut imaginer.

Alain Lancelot (dans la salle) : Je souhaiterais mettre un petit bémol à ce que vient de dire Jacques Rigaud. Je partage totalement son opinion en ce qui concerne l'évolution de la radio par rapport à la télévision. Je crois que la télévision publique est dans une large mesure un très mauvais remake de la télévision privée, même dans l'information. La logique du fait divers constitue l'essentiel du journal télévisé. La télévision publique ne remplit pas son devoir dans une démocratie.

En ce qui concerne la radio, je serais un peu moins euphorique que Jacques Rigaud. Je trouve que souvent en écoutant la radio, nous devons choisir entre marxisme et marché. Nous pouvons avoir la logique du marché dans les stations privées, mais le service public donne souvent l'impression de présenter les choses comme le journal officiel de la gauche socialiste, un peu comme l'ORTF était jadis à sa manière le journal officiel du 
parti gaulliste. Sans doute cette déviation n'est-elle pas la même pour toutes les radios publiques : sur France Info, elle est particulièrement frappante, sur France Inter un peu moins, sur France Culture encore moins, mais l'ensemble est un peu agaçant.

Elisabeth Flüry-Hérard (dans la salle) : Le CSA s'interdit évidemment en toute occasion de prononcer quelque jugement que ce soit sur une radio ou sur une télévision en France. A fortiori, il n'a que peu de choses à dire sur le service public puisque les objectifs du service public de la radio et de la télévision sont fixés par son actionnaire : l'Etat.

Je voudrais simplement rappeler que la conception qui règne en France est celle d'un service public à vocation à la fois généraliste, et, en même temps, un peu plus exigeante au plan culturel. Il y a des chaînes de service public qui sont très pointues : ARTE est une réussite franco-allemande de service public absolument exemplaire. Il n'y a pas qu'ARTE: France 5 et France 4 aussi ont une exigence de service public. A côté, il existe une télévision de service public généraliste. Je voulais rappeler que l'objectif du service public en France peut aussi se rapprocher de l'offre des télévisions privées.

Les termes des débats internes allemands et français me semblent proches. La question allemande de savoir si la réglementation est justifiée par la rareté des fréquences ou par le pluralisme, et si la vérification du respect d'un équilibre de l'information doit s'effectuer par des autorités indépendantes ou par l'Etat, se reflète aussi dans le débat français sur la notion de service public, qui est une notion souple.

Cette souplesse permet d'avoir une définition en fonction des circonstances. Dans les années 1950, on a justifié le service public par la nécessité de construire un réseau qui permettait l'accès de tous à la télévision. Il n'y avait qu'une seule chaîne, et donc la définition du service public était relativement simple. Il fallait que chacun puisse avoir accès à un service qui dépendait de l'Etat puisque le réseau hertzien est un bien public et que ce service devait pouvoir être accessible, y compris dans les régions les plus reculées et montagneuses de la France. Puis progressivement est née cette idée de défense du pluralisme. Comme il y avait un nombre limité de médias, on a pensé que la puissance publique devait intervenir et qu'on ne pouvait pas confier au marché le soin d'organiser la pluralité des points de vue et l'accès de toutes les opinions à l'espace public.

Puis est arrivée la profusion des chaînes : on ne pouvait plus justifier le service public par la nécessité de gérer la pénurie. On a inventé alors une nouvelle conception du service public au travers des missions de service public. Par rapport à la télévision commerciale faite par le marché, il y avait toute une offre de télévision qui devait être proposée et qui ne pouvait pas être financée par le marché. Ainsi on en est arrivé à distinguer les émissions de télévision relevant de missions de service public des émissions de télévision qui pouvaient être diffusées par des chaînes publiques mais qui ne relevaient pas de la mission de service public. Et qui étaient en concurrence frontale avec les chaînes commerciales.

Enfin, on a également inventé cette notion d'équilibre entre le secteur public et le secteur privé. Puisqu'on avait deux chaînes privées de télévision extrêmement puissantes, il fallait qu'en face, il y ait quelque chose qui fasse la balance. On a de la sorte créé ce que j'appelle le double bind ou l'injonction paradoxale adressée à la télévision publique française, c'est-à-dire qu'on lui demande simultanément de faire la même chose que les chaînes privées pour pouvoir les concurrencer et autre chose que les chaînes privées pour 
pouvoir relever effectivement du service public. Naturellement, l'injonction paradoxale rend fou, et c'est la situation actuelle du service public de la télévision.

B. Holznagel : Théoriquement, il est vrai qu'on justifie généralement les activités publiques par une défaillance du marché. Une nouvelle idéologie se propage en Allemagne : celle des sciences économiques. Elles sont devenues la référence : aujourd'hui, il faut être économiste pour être entendu (le juriste a perdu son prestige). Or les économistes nous disent que le facteur décisif est la défaillance du marché ( «market externalities »). Cela dit, il est très difficile de déterminer ce qui relève de la défaillance du marché, quels sont les domaines où s'impose la nécessité d'un service public de l'audiovisuel et quels sont ceux où le marché peut est susceptible d'offrir des services comparables. C'est pour cette raison que l'organisation du marché de l'audiovisuel est soumise à une décision politique.

Or il est important dans ce contexte de rappeler que l'Allemagne est un Etat fédéral qui regroupe 16 Länder. Chacun des ministres-présidents, les chefs de gouvernement de ces 'Etats membres', se préoccupe en priorité de son Land car il souhaite être réélu et être vu dans les télévisions locales. Il soutient donc sa chaîne publique régionale. Celleci offre de surcroît de nombreux postes lucratifs aux députés du Land au sein de ses instances de surveillance. Des intérêts très puissants lient donc aussi les parlementaires et l'audiovisuel public. Enfin, troisième particularité : les présidents des établissements de radiodiffusion de droit public des Länder sont souvent bien plus puissants que les ministre-présidents. Certains sont en effet à la tête d'un établissement couvrant plusieurs Länder, comme ce Mitteldeutscher Rundfunk commun aux Länder de Saxe, SaxeAnhalt et Thuringe ou la ZDF (Zweites Deutsches Fernsehen), société commune à tous les 16 Länder. C'est là qu'il faut chercher la clef de la puissance de l'audiovisuel public allemand : dans le fait que l'Allemagne n'est pas un Etat centralisé.

Ce mode d'organisation ne peut jusqu'ici se maintenir que parce que les programmes du service public se distinguent de ceux de la concurrence privée. Mais il est condamné à terme. La relève des générations va apporter un changement culturel : les nouveaux présidents des chaînes, âgés d'une cinquantaine d'années, se sont appropriés l'économie d'Internet et intègrent déjà les mutations des médias et de la communication dans leur stratégie de développement du service public.

\section{Des médias organisés ou menant une " vie de bohême " ?}

B. de Peretti : A quoi ressemblera le paysage médiatique en 2010 ?

U. Kammann : C'est difficile à prédire, et il faut se méfier des projections qui frisent la conjecture. Je m'en tiendrai pour ma part à la formule de l'éditeur Hubert Burda, patron d'un média classique donc, pour qui les médias numériques mènent actuellement une «vie de bohême ». Tout est en devenir, aucune certitude n'est possible.

Cela dit, on peut déceler une double tendance à l'individualisation comme à l'uniformisation des médias - en tout cas pour une partie des utilisateurs - terme que je préfère à celui de client, car il est plus ouvert. Au vu de la propension des consommateurs à chercher systématiquement le meilleur rapport qualité-prix comme de l'impératif de la réduction des coûts pour les offreurs de services, la massification de l'échange de contenus mènera plus vraisemblablement plutôt à une standardisation de l'offre qu'à une individualisation. Mais, à mon sens, les deux tendances vont évoluer en parallèle. 
Dans ce nouveau monde de communication, il y aura toujours une place pour une offre publique, du moins tant qu'il y aura une volonté politique en ce sens. La question est plutôt de savoir si elle continuera de disposer du même soutien financier. Le service public allemand dispose aujourd'hui de 7 milliards $€$ de redevance. Ce montant est équivalent à ce que dépensent l'Etat fédéral, les Länder et les communes pour la culture (théâtres, musées, bibliothèques, etc.). L'importance du service public en tant que vecteur culturel est donc reconnue.

Je pense pour ma part qu'il en restera ainsi et que sera préservée à l'avenir cette capacité d'intégration qu'il n'a pas encore perdue ; cette mission d'intégration, du moins en tant qu'objectif, sera plus nécessaire que jamais dans un contexte d'individualisation, de fragmentation, voire d'atomisation de la société. Je pense que l'écart se creusera entre ceux qui se satisferont de médias de piètre qualité, à bas prix, et ceux qui se tourneront vers des médias plus onéreux, mais de qualité. La consommation des médias sera plus segmentée.

F. Balle : L'histoire nous apprend que l'offre et la demande évoluent ensemble et s'adaptent. Aucun 'nouveau' média n'en a jamais évincé un autre : le cinéma, la presse, la radio, la télévision et aujourd'hui la Toile, coexistent. Quand la télévision est née, le cinéma s'est cru condamné. Aujourd'hui, en France, il vit très largement grâce à la télévision. La presse est en train de trouver avec la Toile des perspectives inédites dont on ne peut pas dire ce qu'elles seront demain. Mais une chose est sûre : la presse continuera à vivre, elle peut jouer la carte de la complémentarité avec la Toile.

Deuxièmement, on voit les médias se précipiter depuis un quart de siècle vers l'infiniment petit et l'infiniment grand. CNN International n'existe que depuis 1986 et Al Jazeera depuis 1996 seulement. Et on voit, dans la brèche ouverte par CNN et Al Jazeera, une floraison de télévisions qui se veulent transnationales, internationales. Simultanément, on voit des médias locaux jouer de plus en plus la proximité. Cela affecte aussi bien le monde de la presse que celui de la radio, de la télévision et du cinéma.

Troisièmement, on voit se multiplier, en même temps que les modes de distribution ou de consommation, les modèles économiques depuis l'extrême gratuité (qui n'est jamais gratuite car il y a toujours quelqu'un qui paie) jusqu'à ce qui sera extrêmement coûteux : il y a de plus en plus de lettres confidentielles qui coûtent très cher mais qui sont indispensables à quelques centaines de personnes qui, parce qu'elles en ont besoin, sont prêtes à consentir à l'effort financier. Ici, la Toile ouvre des perspectives, car en verrouillant votre système vous pouvez faire payer très cher une revue de presse, des analyses, ce qui existe déjà depuis quelques années dans une version imprimée aussi très onéreuse.

Personne n'avait vu arriver le séisme du numérique et du haut débit. Il y a deux facteurs d'évolution. D'abord, le numérique qui fait que n'importe quel contenu est désormais traduisible sur n'importe quel média - quand vous faites un livre, l'éditeur vous demande de bénéficier de ses droits audiovisuels et sur Internet. La numérisation fédère des médias qui autrefois étaient totalement étrangers les uns aux autres. Ensuite, le haut débit va s'accélérant et permet l'arrivée d'une chose anthropologiquement importante, c'est-à-dire la juxtaposition de langages qui étaient totalement étrangers les uns aux autres, c'est-à-dire le monde de l'imprimé (le monde du XIXe siècle) et le monde de la télévision (celui du XXe siècle). 
On ne sait pas encore utiliser le vocabulaire multimédia, on ne sait pas encore jouer des atouts respectifs de l'écrit, du son et de l'image animée alors que techniquement vous pouvez avoir des programmes qui, selon les cas, vous offrent du texte, une bande son ou une bande vidéo avec des images animées. Je crois que le secteur qui va bénéficier le plus du langage multimédia (mais c'est un pari) lui permettant de jouer sur le texte, le son et l'image animée, est peut-être le secteur de l'information.

Il y a eu une information pour tous grâce aux rotatives au XIXe siècle avec le journal quotidien dont Hegel avait dit: "c'est la prière lä̈que du citoyen moderne ». Il y a eu le divertissement pour tous sous la forme de la télévision dans la deuxième moitié du XXe siècle, qui a pris le relais du cinéma qu'elle a apporté dans tous les foyers. L'ordinateur et tous les terminaux d'aujourd'hui pourront être mieux utilisés pour les problèmes de formation puisque les systèmes de formation sont en difficulté partout dans le monde et qu'on a l'impression que c'est le tonneau des Danaïdes. Il y a beaucoup d'insatisfaction au bout du tuyau, et il y a de plus en plus d'argent mis dans le tuyau. On se dit que peut-être les nouvelles technologies serviront demain à une formation plus égalitaire, comme l'information a pu jouir des rotatives au XIXe siècle et comme le divertissement a pu bénéficier de la télévision au XXe.

B. Holznagel : La décennie à venir sera celle des chaînes publiques car elles entretiennent les meilleures relations avec le monde politique et qu'elles vont continuer à recevoir 7 milliards $€$ de financement. Les autres grands gagnants seront les moteurs de recherche de type Google, qui parviennent à priver la presse et les chaînes de télévision commerciales de recettes considérables. Je pense que la télévision privée va même être démantelée car elle sera rachetée, remodelée, revendue, notamment par les moteurs de recherche ou les entreprises de télécommunications.

Je pense aussi, comme Uwe Kammann, que les chaînes privées vont devenir des fournisseurs de contenu qui mettront sur le marché des contenus de qualité dans le segment premium, ce qui accroîtra leur rentabilité étant donné que le contenu est roi. Mais cette évolution sera le fait d'un grand groupe américain qui ne sera pas du tout régulé et que sa stratégie progressive et discrète de développement imposera sur le marché. Autrement dit, la régulation devra intégrer dans son champ ces nouveaux gate keepers que sont les offreurs de plateformes ou de moteurs de recherche.

Les perdants seront les entreprises de télécommunications. Elles sont soumises à une très forte régulation de la part de Bruxelles, à laquelle il leur est impossible de se soustraire. Je m'attends à ce que les problèmes de concentration verticale que nous avons évoqués soient régulés en dernier ressort par Bruxelles. Pour le compte du Conseil des médias (Medienrat) de la Rhénanie du Nord-Westphalie, qui est une instance de conseil et d'évaluation des politiques médiatiques du Land au sein de l'autorité de régulation régionale LfM, j'ai été amené à étudier plusieurs options pour réguler la concentration verticale. La plus simple serait de transposer à l'audiovisuel les normes applicables aux opérateurs de télécommunications et contenues dans la loi afférente ; elles ont fait leurs preuves. Certes, il n'y aurait alors plus d'auto-régulation de l'audiovisuel, puisque les instances compétentes seraient dès lors la Bundesnetzagentur (dont l'homologue français est l'Arcep) et l'Office fédéral des Cartels.

Mais il semble plus probable que l'intensification de la concurrence empêchera l'intégration verticale. Et comme les entreprises de télécommunication ne sont pas 
familiarisées avec le commerce de contenu et que la seule diffusion d'informations ne suffit pas à assurer la rentabilité, ces dernières seront à mon avis les grandes perdantes des années à venir. Quant aux chaînes privées, elles seront perdantes au profit des moteurs de recherche.

On assistera donc à une sorte de 'remonopolisation' de l'audiovisuel public, mais uniquement en Allemagne et en Grande-Bretagne, où la BBC possède un pouvoir similaire. La situation du marché sera différente en France, tout dépend de savoir s'il est possible d'y endiguer le pouvoir des 'Googles'... 


\title{
Debat $\mathbf{N}^{\circ} \mathbf{2}$
}

\author{
NOUVEAUX DEFIS POUR \\ LA REGULATION ET LE CONTROLE. \\ Quels niveaux et quelles instances?
}



DEBAT $\mathbf{N}^{\circ} \mathbf{2}$

\title{
NOUVEAUX DEFIS POUR LA REGULATION ET LE CONTROLE. Quels niveaux et quelles instances?
}

\author{
EXPOSE INTRODUCTIF : Francis Balle (IFP, Université Paris 2) \\ INTERVENANTS : \\ Dieter Dörr (KEK) \\ Elisabeth Flüry-Hérard (CSA) \\ Jasmin Kundan (Bundeskartellamt) \\ Débat animé par Hans-Jürgen Jakobs (Süddeutsche Zeitung)
}

Face à la convergence des marchés, les régimes parallèles de régulation (télécommunications / audiovisuel) et de contrôle des concentrations (pluralisme des opinions / pluralité des acteurs) ont-ils encore un sens ? Si la question se pose dans les deux pays, la France et l'Allemagne ne l'abordent pas de la même manière, tant les structures institutionnelles et les cultures divergent : centralisation des compétences / polycentrisme, contrôle étatique de l'audiovisuel / contrôle par la société civile organisée, levée récente du monopole public de l'audiovisuel en France, avec constitution d'une autorité de contrôle indépendante, externe aux opérateurs / extension du marché de l'audiovisuel en Allemagne avec extension du contrôle sociétal aux opérateurs privés (Landesmedienanstalten) ...

S'ajoute à cela la double appréciation des médias, à la fois sous l'angle de la régulation sectorielle spécifique et sous celui du droit commun de la concurrence, qui aboutit à un paysage institutionnel d'une haute complexité et débouche sur des choix différents. Deux exemples récents le révèlent : la fusion TPS/CanalSat et l'échec du projet de fusion Springer/ProSieben Sat.1. Dans le premier cas, malgré les avis mitigés de l'ARCEP, du CSA et du Conseil de la concurrence, le ministre de l'Economie, des Finances et de l'Industrie a autorisé la fusion des deux plateformes, conférant à la nouvelle entité une situation de monopole dans la TV à péage. Les autorités indépendantes françaises n'ont qu'une "fonction de gendarme ", et leurs avis sont purement consultatifs. Une telle centralisation des décisions n'est pas envisageable en Allemagne : l'Office fédéral des Cartels est une juridiction; en outre, l'audiovisuel entre dans la compétence culturelle des Länder et y relève en outre de deux sources du droit (droit commun et droit constitutionnel), ce qui se traduit par un double éclairage des questions de concentration. L'Office fédéral des Cartels et la Commission pour l'évaluation de la concentration dans les médias (KEK, gardienne du pluralisme à l'échelon des Länder pour le compte de la société civile), bien que considérant le projet de fusion Springer/ProSiebenSat.1 sous des angles différents, ont estimé qu'il comportait le risque d'une position dominante. 



\title{
EXPOSE INTRODUCTIF
}

\section{La genèse mouvementée de l'idée de régulation en France}

\author{
Francis Balle
}

Pour présenter la situation de la régulation de l'audiovisuel en France, je partirai de deux remarques. La première : à la fin des années 1970, nous nous accommodions parfaitement bien du monopole public sur la télévision. Nous le justifiions par ce qui fait notre idéologie du service public : neutralité, gratuité et une certaine continuité du service, par conséquent un monopole public avec une réglementation précise concernant les quotas d'émissions et les règles concernant le pluralisme. A la fin des années 1970 sont nées quelques radios pirates qui scandalisaient beaucoup les politiques, alors que l'on tolérait tout juste des radios privées qui existaient déjà sur le territoire national, mais avec beaucoup de condescendance, alors qu'elles avaient fait preuve d'un grand professionnalisme et de beaucoup d'esprit d'indépendance et auxquelles nous devions beaucoup, on les appelait les «radios périphériques ». Il y avait donc la radio d'Etat, qu'il fallait absolument honorer de toutes les vertus, et les « radios périphériques » qui nous avaient apporté une bouffée d'oxygène, que ce soit RTL, ici représentée par Jacques Rigaud, ou Europe 1, qui a apporté beaucoup sur le terrain des programmes innovants («Salut les Copains », etc.) tout au long des années 1960.

On s'accommodait de ce monopole auquel on prêtait beaucoup de vertus, mais l'idée d'une nécessaire autorité de régulation faisait petit à petit son chemin, à travers un homme qui a fondé l'Eurovision, Jean Darcy. Il a occupé dans le cadre de la télévision publique française un rôle important : il a fondé l'Eurovision et a choisi le fameux «Te Deum » qui l'illustre. Il a petit à petit convaincu quelques personnes de l'idée qu'il faudrait un jour rompre avec ce monopole, et sans passer d'un monopole public à un monopole privé, instaurer ce qui existait déjà depuis 1924 aux Etats-Unis pour la radio et plus encore depuis 1934 pour la télévision, c'est-à-dire une autorité de régulation.

Deuxième remarque, sur le mot «régulation », pour éviter toute équivoque. Au sens très large, c'est tout ce qui ordonne quelque chose. Mais la régulation peut être le marché, ou la réglementation ; le marché est un mode de régulation des activités humaines, la réglementation également. Les réglementations s'appliquent à tous. Entre les deux, il y a la régulation au sens étroit du terme, c'est-à-dire l'action des autorités de régulation : l'intervention, dans le cadre de la loi, de professionnels et de personnalités indépendantes, lorsque le marché est défaillant (ou déficient, insuffisant, inefficace) et lorsque la réglementation ne peut pas s'appliquer.

L'idée de régulation a fait peu à peu son chemin parmi les juristes à la fin des années 1970 lorsqu'ils ont cessé de prêter toutes les vertus au secteur public dans un contexte où se multipliaient les radios pirates. Mais elle était loin d'être acquise. Pour illustrer l'état d'esprit dans lequel nous étions encore en 1980, en France, je vous donne un 
exemple. Alors que je plaidais auprès d'un homme politique : "Pourquoi ne pas régulariser les radios qui existent ailleurs et qui chez nous sont pirates, dont illégales?», celui-ci m'a objecté : «Vous voulez donc des guérilleros à tous les coins de rue ?! »...

Haute Autorité, puis CNCL, puis CSA

En 1982, on a enfin admis la légitimité des radios locales privées et on les a légalisées. Il a bien fallu instaurer une autorité qui s'est appelée la Haute Autorité de l'Audiovisuel et qui a été confiée à Michèle Cotta, à la fois journaliste, chercheur et professeur. On a justifié l'existence de cette Haute Autorité par deux raisons. La première : il fallait répartir la pénurie, allouer les fréquences à ceux qui les demandaient, moyennant quoi on les obligeait à respecter un certain nombre de contraintes. La seconde : il fallait que ces radios respectent la notion de pluralisme.

En 1984 fut créée Canal +, la première télévision privée en France, mais à péage au forfait, par André Rousselet qui venait de l'Elysée où il était l'un des conseillers les plus proches du Président de le République François Mitterrand. En 1985 sont nées les premières grandes chaînes privées : la Cinq et puis la Six. 1986 fut l'année d'une nouvelle alternance politique, suivie d'une redistribution des cartes avec la création d'une autorité qui prit la suite de la Haute Autorité : la CNCL (Commission Nationale de la Communication et des Libertés) dont le champ d'action était extrêmement large. Elle s'occupait non seulement des radios et des télévisions privées et publiques, mais aussi du secteur des télécommunications, dont on voyait poindre les premiers services à travers le Minitel (qui remonte à 1979/80).

Puis une nouvelle autorité a été créée en 1989 : le Conseil Supérieur de l'Audiovisuel (CSA) qui a vu son champ de compétences réduit au seul secteur de l'audiovisuel. Son mode de désignation a été calqué sur celui du Conseil Constitutionnel, c'est-à-dire : trois personnalités nommées par le président de la République, trois autres par le président du Sénat et trois autres encore par le président de l'Assemblée Nationale ; le Conseil est renouvelable par tiers tous les deux ans. Depuis, cette autorité fonctionne bien ; elle est entrée dans les mœurs.

Or en 1989, lorsque le CSA a été créé, ses neuf membres ont été nommés d'un coup, et pour quatre ans. La sagesse a voulu qu'il y eût parmi ceux-ci deux hauts fonctionnaires (du Conseil d'Etat et de la Cour des Comptes), deux représentants du monde du journalisme (Roland Faure, qui avait créé France Info, et Geneviève Guichenet, qui avait présenté le «Soir 3 »), deux personnalités venues du monde de la production (Daisy de Gallard et Igor Barrère), deux universitaires dont j'étais, puis enfin le président de la fédération des associations familiales de France. Les équilibres politiques étaient assez satisfaisants.

Ce qui a créé réel un lien entre nous, c'est le fait que nous avons été traités en mai 1989 «d'ayatollahs » par Télérama, le seul hebdomadaire français à ne pas aimer ce dont il parle : la télévision, parce que nous avions estimé que les films de charme ne devaient pas être diffusés à l'écran avant $22 \mathrm{~h} 30$. Nous avons été désavoués par le Ministre de la Culture et de la Communication de l'époque qui a imposé la diffusion de ces films à des horaires trop précoces à nos yeux. Cela a soudé notre équipe.

Ensuite, l'institution est entrée dans les mœurs avec cette difficulté qu'elle a toujours : tout le monde croit que le CSA a la possibilité de dire aux chaînes publiques ce 
qu'elles doivent faire, or cela lui est interdit ; et tout le monde s'imagine que, vis-à-vis des chaînes privées, le CSA manque d'autorité, alors qu'il assortit les autorisations données aux chaînes d'utiliser le domaine public - c'est-à-dire les fréquences -, d'un certain nombre d'obligations particulières au respect desquelles il veille ensuite.

\section{Aujourd'hui, deux autorités distinctes pour}

l'audiovisuel privé et les télécommunications : CSA et ARCEP

Voilà comment l'idée de régulation a fait difficilement son chemin, et comment petit à petit le CSA a trouvé sa place entre d'un côté le droit de la concurrence dont je pense qu'il prend de plus en plus d'importance, dès lors qu'un acteur majeur se met à jeter son dévolu sur les droits d'évènements qui rapportent beaucoup d'argent, les droits sportifs ou le cinéma, et puis d'un autre côté l'ARCEP qui a pris la suite de l'ART (créée en 1996), c'est-à-dire l'institution qui opère la régulation des activités de télécommunication. Certains avaient pourtant pensé que celle-ci était inutile et qu'il aurait mieux valu ne créer qu'une seule instance, compétente pour les télécommunications et l'audiovisuel, comme aux Etats-Unis, au Canada ou en Grande Bretagne... 


\section{DISCUSSION}

Hans-Jürgen Jakobs : Merci beaucoup pour cette immersion dans l'histoire récente des médias et de leur régulation en France. Aujourd'hui, la multiplication rapide des services, des acteurs, des modes de distribution comme des récepteurs place les instances de régulation traditionnelles face à de nouveaux défis. Comment celles-ci peuvent-elles encore effectuer un contrôle approprié ? Que doit-on réformer et/ou améliorer?

Elisabeth Flüry-Herard : Je ne m'exprime pas au nom du CSA mais en mon nom personnel, car les problèmes dont nous allons parler n'ont pas fait l'objet de prise de position officielle du CSA. Sur un certain nombre de points, les questions qui se posent aujourd'hui en Allemagne sont en avance par rapport à la réflexion sur la régulation en France, malgré le fait que le rapport de la Commission présidée par Alain Lancelot a exposé la problématique de façon claire et remarquable.

En France, c'est le ministère de l'Economie et des Finances qui est l'autorité de régulation compétente en matière de concentrations dans le champ des médias. Il prend cette décision sur la base d'un avis du Conseil de la Concurrence lorsqu'il est saisi, et qui lui-même a recueilli les avis du CSA et éventuellement de l'ARCEP. C'est un système de droit commun, très classique, dans lequel la décision est prise en fonction des principes du droit de la concurrence, le CSA se contentant théoriquement de donner un avis sur la base du pluralisme.

Le CSA gère l'offre (réduite) de fréquences, alors que la régulation allemande considère le rôle de l'audiovisuel dans la formation de l'opinion

Dans les faits - nous avons eu récemment le cas d'une très grosse concentration, celle de CanalSatellite et de TPS - quand le CSA donne son avis, il le donne aussi sur la définition des marchés pertinents. C'est l'autorité sectorielle compétente. Même s'il donne son avis au nom du pluralisme, il va donc faire bénéficier les autorités de la concurrence de son expérience sectorielle. Mais c'est seulement le premier niveau, car dans le cas d'une grosse concentration audiovisuelle, il va y avoir un examen non seulement sous l'angle du droit de la concurrence mais également au titre de la Loi de 1986 relative à la liberté de la communication.

Il faut rappeler dans ce contexte que le hertzien terrestre reste prédominant en France. Il est synonyme de pénurie de fréquences, ce qui a pour conséquence que l'un des rôles essentiels du CSA est d'attribuer les fréquences selon les critères définis par la loi. Et quand le détenteur de l'autorisation d'exploiter une fréquence change, le CSA a son mot à dire. Et lorsqu'il y a concentration dans le secteur des médias, c'est bien une autorisation qui change de main. Au nom de la pénurie des fréquences, le CSA s'est donc vu attribuer un rôle considérable pour approuver ou non une concentration. C'est le premier rôle spécifique que lui confère la loi de 1986.

Le deuxième est un dispositif anti-concentration spécifique. Celui-ci reste basé sur la notion de la gestion de l'offre de fréquences et pas du tout sur la conception de la 
demande ou de l'audience, comme c'est le cas en Allemagne, où on considère le rôle de l'audiovisuel dans la formation de l'opinion, et où on va faire en sorte qu'un opérateur ne puisse pas avoir le contrôle d'une audience majoritaire. En France, comme il y a peu de fréquences, il ne faut pas qu'une même personne tienne un grand nombre de fréquences. A peu près tout le système de la loi 1986 est fondé sur cette idée de limiter le nombre de fréquences détenues par un opérateur, même si les audiences sont limitées.

Le rôle du CSA en cas de concentration peut être important, comme dans le cas de la fusion TPS / CanalSat. L'opération de concentration concernait le secteur de la télévision payante. En France, elle s'est fortement développée avec à la base deux distributeurs : le groupe Canal+ d'une part et le groupe TPS d'autre part, TPS étant une émanation de TF1 et de M6 (RTL Group). Or ces deux plateformes satellite n'arrivaient pas à trouver la rentabilité et ont donc cherché à fusionner.

La problématique concurrentielle était extrêmement complexe parce que la nouvelle entité, Canal+ France, avait des positions fortes à la fois sur le marché aval, c'est-à-dire la distribution de télévision payante au public, et sur le marché amont, c'est-à-dire l'acquisition des droits de diffusion de cinéma ou de sport, et enfin, sur le marché intermédiaire, c'est-à-dire l'offre de chaînes de télévision pour les distributeurs. Autrement dit, une concentration verticale extrêmement forte qui est caractéristique de la situation française.

Le processus a été le suivant: d'abord un avis du CSA et un avis de l'ARCEP, ensuite l'avis du Conseil de la Concurrence, ensuite la décision du Ministre qui a autorisé la concentration en échange de 58 engagements. Au titre de ces derniers, la chaîne TPS Star, qui est une chaîne premium à la fois sur la TNT et sur le satellite, devait être dégroupée, c'est-à-dire mise à disposition des autres opérateurs, distributeurs de télévision payante.

Du point de vue du droit de la concurrence, l'opération est achevée, mais elle ne l'est pas du tout du point de vue de CSA, parce que, comme il y a une licence hertzienne en jeu (TPS Star a une licence hertzienne), le CSA doit maintenant décider si oui ou non il va laisser cette licence hertzienne à TPS Star et donc au nouveau groupe.

La situation française est donc relativement paradoxale : il y a eu un énorme travail des autorités de concurrence sur la fusion TPS / Canal+ et au final, l'instance de régulation de l'audiovisuel peut dire oui ou non sur le fait que TPS Star, qui est une partie très importante de l'opération de concentration, va garder ou non une licence hertzienne. On pourrait dire que, à partir du moment où les autorités de la concurrence se sont prononcées, le CSA n'a rien à dire. Ce serait parfaitement illégal de dire cela puisque le CSA, de par la loi, doit avoir un avis sur le sujet - fondé cette fois-ci sur le pluralisme et non pas sur la préservation du marché et sur la concurrence.

Je vous ai cité ce cas pour vous montrer à quel point, malgré tout, la question des interactions entre la garantie du pluralisme, dont est chargé le CSA, et la préservation de la concurrence au sein d'un marché dont est chargé de Conseil de la Concurrence, n'est pas une question abstraite.

Un autre exemple est la mission de règlement des conflits dont est chargée le CSA, par exemple en cas de conflit entre un distributeur et un éditeur de télévision. C'est typiquement une mission de régulation à la frontière du pluralisme et du marché dans la problématique de la concentration verticale qui caractérise l'audiovisuel. Celle-ci est un 
problème moins important pour les autorités de la concurrence, mais elle est un très gros problème sous l'angle du pluralisme. Les interactions n'en demeurent pas moins délicates.

Pour terminer, on voit bien, aujourd'hui, qu'il y a des fréquences qui sont attribuées au CSA et d'autres qui sont attribuées à l'ARCEP. Le jour où on fera de l'audiovisuel sur des fréquences attribuées par l'ARCEP, qui s'occupera du pluralisme ? L'avenir de l'interaction des autorités de régulation comme des droits applicables, reste à définir.

En Allemagne, multiplication des compétences et des instances du fait du fédéralisme

H.-J. Jakobs : Le système français est moins complexe que le système allemand. En ce qui concerne le dispositif, coexistent en Allemagne l'Agence fédérale des réseaux (Bundesnetzagentur) qui compte les télécommunications parmi ses attributions, l'Office fédéral des Cartels (droit commun des concentrations), 15 autorités de régulation des médias régionales (une Landesmedienanstalt par Land, sauf dans le cas de Berlin et du Brandebourg, qui ont une instance commune), de même que la Commission d'évaluation de la concentration des médias (Kommission zur Ermittlung der Konzentration im Medienbereich, KEK).

Par ailleurs, les 11 établissements de radiodiffusion de droit public allemands possèdent chacun une sorte de conseil de surveillance, organe de régulation interne (Rundfunkrat). Quant à l'évaluation de la redevance, elle relève d'une autre instance encore : la Commission d'évaluation des besoins de financement des établissements publics (Kommission zur Ermittlung des Finanzbedarfs der Rundfunkanstalten, KEF).

En ce qui concerne les compétences réglementaires, il faut distinguer entre l'échelon des Länder, souverains en matière d'audiovisuel, et l'échelon fédéral, compétant pour d'autres questions, notamment le droit de l'économie. Existe-t-il en France des éléments dont l'Allemagne pourrait s'inspirer?

Jasmina Kundan : Il semble qu'en France, les décisions soient plus centralisées, tant sous l'angle de la concentration que du pluralisme. Certes, dans la structure fédérale de l'Allemagne, la structure institutionnelle est plus complexe, mais à ma connaissance, les décisions des différentes instances n'en convergent pas moins. On peut néanmoins se poser la question de l'efficience de la coexistence d'autant d'instances, bien que le fédéralisme présente par ailleurs un certain nombre d'avantages.

A l'Office fédéral des cartels, nous partons du principe que la protection de la concurrence permet de garantir indirectement la liberté d'opinion, car où il y a concurrence, il y a forcément pluralisme.

Mais cela ne signifie pas que nous pensions que le contrôle sectoriel des médias soit superflu car il existe des domaines où les deux approches - celle du marché ou des opinions - ne se recoupent pas complètement. Les autorités de régulation des médias (Landesmedienanstalten) des Länder s'intéressent à la croissance interne des groupes audiovisuels. L'Office fédéral des Cartels n'a aucune compétence sur ce point, et considère donc les médias sous l'angle des structures oligopolistiques qui, elles, ne sont pas prises en compte par les autorités des Länder.

Le système Français présente des avantages, mais du fait de la structure fédérale de l'Allemagne, il n'est pas transposable. Mais le système allemand n'est pas forcément moins bon; l'éclairage simultané d'une problématique par deux types d'institutions n'est peut-être pas une mauvaise chose non plus. 
Dieter Dörr : Le contrôle du pluralisme d'opinions et celui de la pluralité des acteurs sur le marché sont deux tâches que distinguent le droit allemand et européen. Et il est judicieux d'avoir deux instances de contrôle distinctes. Cela étant, certains se demandent si le contrôle des médias ne devrait pas échapper largement aux compétences de l'Office fédéral des Cartels.

Les Länder exercent le contrôle du pluralisme, le Bund, celui de la concurrence

Je ne partage pas cet avis, étant donné que, au niveau du droit de la concurrence, s'est constituée une jurisprudence permettant d'améliorer le contrôle des médias. L'Allemagne présente évidemment les avantages tout comme les inconvénients d'un système fédéral, l'audiovisuel relevant des Länder sous l'angle du pluralisme, de l'échelon fédéral sous celui de la concurrence...

H.-J. Jakobs : Que faudrait-il changer pour gagner en efficience ?

D. Dörr : La question a déjà été amplement débattue en Allemagne. Mais les axes possibles de réforme achoppent sur des crispations politiques. Il en va ainsi de la création d'une instance de régulation des médias regroupant l'ensemble des Länder pour toutes les questions d'autorisation ou de pluralité à l'échelle de l'ensemble du pays. Cette possibilité a déjà été évoquée à de nombreuses reprises. Les Länder s'en sont rapprochés en partie, par l'institution de la Commission de l'évaluation de la concentration (Kommission zur Ermittlung der Konzentration im Medienbereich, KEK), en charge des questions relatives à la pluralité des opinions, ce qui inclut la prise en considération des segments pertinents voisins de l'audiovisuel.

C'est sur cette base que la KEK a décidé au début 2006 de ne pas autoriser la fusion Springer/ProSiebenSat.1. Ses décisions sont en principe contraignantes, mais la Conférence permanente des Directeurs des Landesmedienanstalten (la KDLM) peut casser une décision de la KEK. Il conviendrait également d'améliorer la coopération entre la KEK et les opérateurs audiovisuels. La KEK ne peut en effet entrer en contact avec les détenteurs d'une licence que par l'intermédiaire de la Landesmedienanstalt qui l'a délivrée.

Victor Henle (dans la salle): J'aimerais apporter une précision sur l'articulation entre ces différentes instances.

La KEK est une instance commune aux Landesmedienanstalten et composée d'experts externes. Elle est l'émanation de celles-ci et agit pour leur compte dans les domaines dépassant leurs compétences territoriales : concrètement, la KEK recense les parts de marché des opérateurs sur l'ensemble du territoire et évalue si une demande d'autorisation ou un projet de fusion respecte les critères de concentration applicables aux médias, c'est-à-dire respecte le principe de pluralité. La Landesmedienanstalt concernée par le dépôt de candidature est alors chargée d'appliquer l'avis de la KEK. Dans le cas du projet de fusion Springer/ProSiebenSat.1, pas moins de trois Landesmedienanstalten étaient concernées.

Ce qui rend les processus de décision aussi complexes, c'est que la KEK ne dispose pas de pouvoir discrétionnaire, les Landesmedienanstalten ne le lui ayant pas transféré. Elles l'ont conservé et l'exercent, sur les questions dépassant leurs compétences territoriales, via la conférence permanente des directeurs des Landesmedienanstalten, qui ont charge de magistrat : la KDLM. Celle-ci doit valider les avis de la KEK et peut les invalider, à la majorité des trois quarts. 
D. Dörr : Il est vrai que cette articulation est d'une haute complexité, et demande à être optimisée, de même d'ailleurs que la coopération entre la KEK et l'Office fédéral des Cartels. Elle souffre en effet d'une asymétrie : l'obligation faite aux Landesmedienanstalten, dont la KEK est l'émanation, de coopérer avec l'Office fédéral des Cartels est totale ; il n'en va pas de même dans l'autre sens. Cela n'entrave cependant pas la coopération au quotidien.

La régulation sectorielle allemande repose en partie sur des concepts flous, comme celui de " position dominante dans l'opinion »

Il faut ajouter à cette complexité institutionnelle le flou qui entoure certains concepts juridiques propres au domaine des médias, comme celui de "position dominante dans l'opinion » (vorherrschende Meinungsmacht). Mais il n'en va pas autrement du droit de la concurrence avec son concept de "position dominante sur le marché » (marktbeherrschende Stellung). L'opinion dominante est un concept qu'a défini le Tribunal constitutionnel fédéral. Il laisse suffisamment de marges d'appréciation à la KEK pour prendre en considération les mutations des médias. Ce concept est fondé sur la télévision, ce qui me semble judicieux. Certains pensent qu'il faudrait radicalement élargir son approche et généraliser le contrôle à l'ensemble des médias. Mais en l'absence de proposition concrète, il vaut mieux s'en tenir à l'état actuel du droit et l'étendre progressivement à l'ensemble des nouveaux médias.

J. Kundan : La coopération entre l'Office fédéral des cartels et la KEK fonctionne de mieux en mieux. Peut-être cette impression vient-elle de ce que nous avons moins d'obligations vis-à-vis de la KEK qu'elle envers nous. Toutefois, certains acteurs cherchent à mettre à profit nos divergences de vues déterminées par les deux sources du droit dont nous tirons nos compétences. Il n'en reste pas moins que la confrontation de deux approches différentes d'une même problématique est fructueuse.

Mais j'aimerais revenir sur les différences systémiques entre la France et l'Allemagne. Chez nous, les différentes instances ou autorités ne se contentent pas de donner des avis ; elles sont habilitées à prendre des décisions. L'Office régional ou fédéral des cartels est statutairement indépendant de l'exécutif, quel que soit le ministre de l'Economie du Land ou du Bund. A ce propos, que se passe-t-il en France quand le Conseil de la Concurrence propose au ministre un avis différent du CSA ? Quel avis le ministre décide-t-il de suivre ?

H.-J. Jakobs : J'ajouterais une autre question : Pensez-vous qu'il soit souhaitable que le contrôle économique et le contrôle des médias soient gérés par deux institutions différentes?

E. Flüry-Hérard : Dans une opération de concentration, il peut même y avoir trois avis, quand l'ARCEP est également consultée. Le ministre français de l'Economie et des Finances prend sa décision en toute liberté et choisit ce qui lui semble bon dans chacun des avis, l'avis étant simplement consultatif, il ne lie pas le ministre.

En ce qui concerne la coexistence de deux institutions, l'une chargée du fonctionnement de la concurrence sur un marché et l'autre chargée des questions de pluralisme, c'est effectivement la situation française : le CSA défend le pluralisme et le Conseil de la Concurrence préserve la concurrence. Le CSA prendra par exemple la défense du petit concurrent des géants des médias parce que, si son existence n'est pas un problème 
pour le fonctionnement du marché, sa disparition peut être un problème pour le pluralisme. Nous essayons de faire cette distinction. Il y a deux droits, deux routes parallèles.

\section{Comment aborder les tendances à la concentration au sein de l'UE ?}

H.-J. Jakobs : Un monopole géant a pourtant vu le jour en France dans le domaine de la télévision payante. Les deux anciens concurrents n'existent plus sous la même forme qu'auparavant, ils forment désormais une unité. Et quand on y regarde de plus près, l'essentiel du capital appartient à des géants, comme le groupe M6 qui appartient à Bertelsmann. Cette concentration présente le risque d'une entente sur d'autres marchés. Ce n'est pas un résultat particulièrement positif du contrôle des médias en France !

E. Flüry-Hérard : Il s'agit d'une décision du ministre des Finances qu'il n'appartient pas au CSA de commenter. Simplement, en France, cette concentration ne concerne pas la presse, puisque les groupes concernés, à l'exception de M6 lié à Bertelsmann, ne sont pas présents dans la presse, ni dans la télévision payante. Lagardère l'est effectivement, mais avec une position très minoritaire.

Le fait qu'il n'y ait qu'un seul opérateur de télévision payante n'est pas réservé à la France : c'est le cas en Italie ou en Grande-Bretagne. Le Conseil de la Concurrence, institution totalement indépendante, a pensé avant même la décision du Ministre des Finances, que d'avoir une seule offre de télévision payante liée à tous les programmes possibles peut être aussi un plus pour le consommateur.

H.-J. Jakobs : En Allemagne aussi, nous avons connu deux cas de monopoles de télévision payante : MSG, une coopération entre Deutsche Telekom, les groupes Bertelsmann et Kirch dans les années 1990, puis une autre entre Kirch et Bertelsmann, au sujet de laquelle Bruxelles a déterminé que cela ne relevait pas directement de sa compétence.

Pensez-vous qu'il y ait un risque que nous revenions à un tel degré de concentration dans le domaine de la télévision payante en Allemagne? Les deux cas précités ont certes été traités à l'échelle européenne mais il est possible que de nouveaux cas apparaissent et qu'ils soient cette fois-ci du ressort de l'Office fédéral des Cartels...

J. Kundan : C'est un domaine très complexe. Jusqu'à présent, Premiere était le principal opérateur de télévision payante en Allemagne. Avec les droits de retransmission du football, Arena se positionne en concurrent sur le marché de la télévision payante. Cette situation nous laisse une impression mitigée car elle stimule l'intégration verticale avec les opérateurs de réseau, c'est-à-dire avec Unity Media en Rhénanie du Nord-Westphalie et en Hesse. Elle risque également d'inciter les grandes chaînes jusqu'ici gratuites à développer leur offre dans le segment à péage, ce qui devrait dynamiser la concurrence sur le marché des contenus payants. L'évolution est plus difficile à prévoir dans les segments en amont et en aval, les relations entre Unity Media et Arena étant relativement tendues.

H.-J. Jakobs : M. Dörr, certains conservateurs reprochent à la KEK d'avoir empêché une entreprise allemande, les éditions Axel Springer AG, de racheter le groupe ProSiebenSat.1Media AG, le plus gros opérateur de TV privée en Allemagne - au risque de le laisser tomber dans l'escarcelle du groupe Mediaset de Milan. Or ce groupe appartient à l'homme politique Silvio Berlusconi. Que répondez-vous à cela ?

D. Dörr : Je me suis toujours étonné de ce reproche, tout comme le président de l'Office fédéral des Cartels, d'ailleurs. Il faut souligner que, dans les débats, on fait 
comme s'il s'agissait d'une entreprise allemande ; or le capital de ProSiebenSat.1Media AG est étranger.

Face à cela, que sommes-nous censés faire ? Créer une réglementation spécifique pour les entreprises de l'UE établies chez nous ? Seul le législateur serait habilité à adopter des dispositions particulières... Mais les voix critiques oublient que nous agissons au sein du marché communautaire, ce qui exclut tout traitement particulier pour les entreprises des Etats membres. L'abondante jurisprudence de la Cour européenne de justice est là pour nous le rappeler au besoin. Il ne revient à la KEK que d'apprécier si la présence d'une entreprise sur le marché allemand, d'où qu'elle vienne au sein de l'UE (de France, d'Allemagne, d'Italie ou de Pologne...), amène une position dominante ou non dans l'opinion allemande.

Contrairement à l'Office fédéral des cartels, nous ne contrôlons pas la position dominante sur un marché mais dans l'opinion. La concurrence des marchés et des entreprises au sein du marché communautaire relève de la Commission. A l'échelon de l'espace public allemand, la mission de la KEK est de veiller à la pluralité ou à la concurrence des opinions ; son contrôle est préventif, la mission de notre Commission étant de veiller au bon fonctionnement du processus de formation de l'opinion. Toute disposition que nous prendrions au-delà de cette mission dépasserait les compétences que nous a confiées le législateur.

H.-J. Jakobs : Sur le territoire allemand prévaut certes une stricte séparation entre les médias et le pouvoir politique. Mais le droit allemand semble totalement impuissant face aux risques de collusion d'intérêts 'importés' de l'étranger, comme dans le cas du groupe médiatique de Silvio Berlusconi, personnalité politique s'il en est. Il n'existe vraiment aucune disposition permettant de prendre cela en considération ?

D. Dörr : Une remarque d'abord : les licences ne sont pas délivrées par la KEK, mais par les Landesmedienanstalten. C'est donc à elles de veiller à l'indépendance des médias. Bien sûr, la KEK est susceptible de donner son avis si elle considère qu'un candidat ne respecte pas le principe constitutionnel de la séparation entre médias et pouvoir politique. Elle l'a fait à de nombreuses reprises. En réalité, la question que vous soulevez est celle-ci : ce principe inscrit dans la Loi fondamentale allemande peut-il ou doitil être appliqué à des opérateurs étrangers ? Mais cette question relève de la quadrature du cercle...

Cela dit, au sein de l'UE, ce principe constitutionnel est propre à l'Allemagne. Et il a pour corollaire la garantie du pluralisme, ainsi que l'a rappelé dans son abondante jurisprudence le Tribunal constitutionnel fédéral : la Loi fondamentale interdit que la télévision allemande appartienne à l'Etat ou soit aux mains d'un seul groupe représentatif de la société. L'application concrète de ce principe peut se révéler délicate dans certains cas...

H.-J. Jakobs : Il y a quelques années, Silvio Berlusconi était présent sur le marché télévisuel français, avec Jérôme Seydoux et d'autres. Il n'était alors certes pas encore au pouvoir, mais il côtoyait François Mitterrand. S'il revenait sur le marché français, comment cela serait-il perçu du point de vue de la régulation ? Quelles sont les règles concernant les investissements d'entreprises étrangères sur le marché télévisuel ?

E. Flüry-Hérard : Il faut distinguer les étrangers communautaires et les étrangers non communautaires. Un ressortissant de l'UE est traité strictement de la même manière 
qu'un Français. C'est la raison pour laquelle une des grandes chaînes de télévision française, M6, est contrôlée par RTL Group. En revanche, s'il s'agit d'un étranger non communautaire et s'il s'agit d'une autorisation hertzienne, une règle dit que l'étranger ne doit pas contrôler une chaîne et ne doit pas posséder plus de $20 \%$ de son capital. Autrement dit, un Européen a exactement les mêmes droits qu'un Français.

H.-J. Jakobs : En France, aucun actionnaire ne peut détenir plus de $49 \%$ du capital d'un opérateur. Ce modèle dit «du consortium » a existé en Allemagne aussi, avant d'être remplacé en 1996 par un modèle où le critère décisif est désormais la part d'audience et non plus la limitation de la part de capital. La France s'apprête-t-elle à réformer cette disposition?

E. Flüry-Hérard : La participation maximale de $49 \%$ est très critiquée, parce que, quand il s'agit d'une entreprise cotée en bourse, connaître la détention exacte du capital n'est pas une évidence. Par ailleurs, même avec $49 \%$ seulement, vous contrôlez une entreprise de télévision. L'intérêt de cette règle en termes de pluralisme fait débat. Certains ont même proposé sa suppression.

\section{Contrôle de l'audiovisuel par l'Etat ou par la société civile ?}

H.-J. Jakobs : Le CSA est également en charge des chaînes publiques. Les objectifs sont fixés par l'Etat mais il possède une fonction de contrôle. Pourriez-vous préciser sa mission?

E. Flüry-Hérard : Sa mission n'est pas de fixer les objectifs du service public. Ceux-ci sont fixés par un contrat entre l'actionnaire du service public, c'est-à-dire l'Etat, et ses sociétés.

En revanche, la compétence du CSA est de surveiller l'application de la réglementation qui s'applique à toutes les chaînes et qui est, grosso modo, dérivée de la Directive Télévisions sans Frontières en France comme en Allemagne. Nous sommes les 'gendarmes' de l'application de la réglementation française, elle-même dérivée de la réglementation européenne pour la protection de l'enfance, la défense du pluralisme, les règles sur la publicité, etc.

D. Dörr : En Allemagne, la Constitution interdit que l'audiovisuel public appartienne à l'Etat. L'approche allemande d'après 1945 est une réaction à l'instrumentalisation de la télévision par le régime nazi. On a donc développé un modèle où l'audiovisuel appartient à la collectivité des citoyens. C'est elle qui contrôle les établissements publics, par l'intermédiaire d'une sorte de conseil de surveillance composé de représentants de la société civile organisée. Le contrôle s'effectue donc en interne.

Néanmoins, ce contrôle a parfois des limites, notamment lorsque la coopération avec des annonceurs au niveau de la production débouche sur une pratique illicite de placement de produit. Ces cas révèlent la nécessité de rendre le contrôle de l'audiovisuel plus efficient. S'il ne peut être question de réorganiser le service public pour le soumettre à un contrôle institutionnel externe, certains domaines pourraient, en revanche, être régulés en externe, comme la protection des mineurs. Elle laisse à désirer dans le public comme le privé, et pourrait donc donner lieu à une régulation indépendante du statut des opérateurs. Mais une réforme en ce sens présente quelques difficultés.

H.-J. Jakobs : Les chaînes publiques étant aussi des acteurs économiques, quelle est l'approche de l'Office fédéral des Cartels ? 
J. Kundan : Les établissements de radiodiffusion de droit public échappent au droit de la concurrence, puisqu'ils ont le statut de collectivités publiques et sont financés par la redevance. Cela dit, l'Office fédéral des Cartels suit attentivement le marché des médias. Et tout particulièrement celui de la publicité télévisée qui joue un rôle considérable pour le segment de la télévision gratuite.

Sur ce dernier, les établissements publics ont une position qui serait considérée comme dominante s'il s'agissait d'une SARL par exemple. Mais du fait de leur statut d'exception, le droit commun ne s'applique pas à eux. Et je ne pense d'ailleurs pas qu'il faille lever cette dérogation. Il n'en va pas de même lorsqu'ils développent des activités commerciales ou créent des filiales de statut privé. Dans ce cas, ils entrent dans la même catégorie que les hôpitaux, par exemple, qui appartiennent certes à des institutions publiques mais qui exercent sous le statut d'une GmbH (une SARL). De ce fait, ils sont soumis au régime commun du droit des cartels et du contrôle des fusions.

H.-J. Jakobs : Autre défi pour le droit allemand : les opérateurs de réseaux commencent à investir le segment des contenus, par exemple en rachetant des chaînes de télévision. Comment le législateur prend-t-il en compte cette évolution ?

J. Kundan : Je pense que, dès lors qu'il ne s'agit pas de croissance interne, le droit de la concurrence s'applique. Nous avons ainsi interdit le rapprochement entre Arena et Unity Media. Mais en l'état actuel du droit, l'application du droit des fusions à un opérateur de réseaux désireux de racheter un opérateur de programmes reste délicate du fait de l'appartenance de ces acteurs à deux domaines du droit : le droit public (qui s'applique aussi aux chaînes privées) et le droit de la concurrence (qui s'applique aux télécommunications en cas de fusion). L'état actuel est certes imparfait, mais j'estime à titre personnel qu'il vaut mieux le faire évoluer en douceur plutôt que de mettre en place trop de réglementations.

E. Flüry-Hérard : En France, le CSA n'est pas juridiquement compétent pour ces questions. Et si par exemple France Télécom, qui est aussi un opérateur de distribution ADSL, envisageait d'acheter les droits du football français, je ne vois ce que pourrait faire le CSA, sinon faire connaître ses inquiétudes dans un communiqué. Il l'avait fait voici cinq ans, lorsque Canal+ avait acheté l'intégralité des droits du football. Il y a eu une action devant le Conseil de la Concurrence. Ce dernier était intervenu, à la suite de quoi il y a eu une médiation et un nouvel appel d'offres. L'instance compétente, en France, est le Conseil de la concurrence.

Werner Schwaderlapp (dans la salle) : Je voudrais revenir sur ce qu'a dit Dieter Dörr au sujet du contrôle de l'audiovisuel public et apporter une réflexion contradictoire. Le contrôle de l'audiovisuel public doit être effectué par la société dans la mesure où aucun autre acteur ne peut remplir cette fonction. Concrètement, cette représentation de la société est confiée aux organes internes de surveillance des établissements de radiodiffusion. Dans ces organes règne un état d'esprit particulier que j'avoue avoir contribué à entretenir à l'époque en qualité de membre de la direction et que Dieter Dörr aurait lui aussi entretenu s'il avait occupé un poste de management dans le service public.

Cet état d'esprit se caractérise par une grande fierté et par une tout aussi grande angoisse existentielle. L'organe de surveillance nourrit une grande fierté du fait d'être autorisé à contrôler des programmes de qualité ; il s'enorgueillit donc de laisser l'exécu- 
tif de l'établissement agir à sa guise, ce qui en fait un complice. Mais si le service public n'obtient pas de hausse de la redevance, ou si le législateur n'accorde pas à l'établissement l'autorisation de se diversifier dans les médias en ligne par exemple, alors surgit immédiatement l'angoisse existentielle - celle de voir le service public même remis en question.

Les Britanniques ont constaté les mêmes mécanismes psychologiques à la BBC et décidé d'organiser une double représentation de la société, donc de lui faire exercer le contrôle à la fois en interne, un peu comme en Allemagne, et par une nouvelle instance, externe. Celle-ci porte un regard extérieur et peut donc considérer les événements avec plus de recul, c'est-à-dire notamment réfléchir aux missions de service public.

J'évoque cet exemple parce que l'Allemagne pourrait s'en inspirer pour moderniser la régulation de l'audiovisuel public. Car notre mode de contrôle interne est une raison structurelle pour laquelle la question des missions du service public de l'audiovisuel est si peu abordée. Le modèle double, britannique, permettrait des gains d'efficience, sans modifier la logique inhérente au système allemand de régulation qui est établi sur la représentation de la société civile. Pour le secteur privé aussi, puisque les membres des Landesmedienanstalten sont eux aussi choisis au titre de cette représentation. La question de savoir comment articuler les modes institutionnels des contrôles pour le public et le privé reste bien sûr en débat. Mais il me semblait important de rappeler ici que le contrôle de l'audiovisuel exercé par la collectivité reste purement théorique.

\section{Le droit de la concurrence est un préalable pour préserver le pluralisme.}

Pourquoi ne pas lui soumettre entièrement la régulation des médias?

Jürgen Heinrich (dans la salle) : Les participants de ce débat ont visiblement choisi de se cantonner à l'état actuel du droit. Mais le droit peut s'adapter à une situation évolutive. La Commission des monopoles, ce conseil scientifique placé auprès de l'Office fédéral des Cartels, propose par exemple de considérer l'attribution d'une licence sous l'angle de la procédure des fusions. Dès lors, c'est l'Office fédéral des Cartels qui jugerait en dernier ressort. Qu'en pensent l'Office et la KEK ?

J. Kundan : Je ne vois pas la nécessité de modifier l'architecture d'un système de régulation qui a fait ses preuves. En outre, la Loi fondamentale confie les compétences en matière d'audiovisuel aux Länder, et il n'y a aucune raison d'y changer quoi que ce soit.

D. Dörr : En effet, ces questions ne relèvent pas du droit commun, mais du droit constitutionnel. La Cour de Karlsruhe a rappelé maintes fois que la garantie du pluralisme relève des législateurs des Länder. Et le président de l'Office fédéral des cartels a fait remarquer qu'il serait absurde de transférer ces compétences dans le champ du droit de la concurrence qui relève, lui, de l'échelon fédéral. Mêler les deux échelons aboutirait immédiatement à un conflit entre les sources du droit et leur objet respectif. C'est pour la même raison que le droit européen distingue lui aussi clairement entre pluralisme et concurrence.

Je suis bien entendu d'accord avec Jasmin Kundan et son Office sur le fait que le droit de la concurrence est un préalable à l'émergence du pluralisme. Il s'agit toutefois d'une condition nécessaire, et non suffisante, le pluralisme, qui est au fondement de la démocratie, requerrant une garantie particulière. Je ne vois nullement le Tribunal constitutionnel fédéral changer de doctrine, ni ses homologues français, italien, espagnol ou 
portugais non plus d'ailleurs. Et je m'inscris en faux contre ces propositions de soumettre le droit spécifique du pluralisme au droit commun de la concurrence, que formulent un certain nombre d'experts ultra-libéraux comme ce Conseil scientifique placé auprès du ministère fédéral de l'Economie.

Sur la question du fonctionnement trop peu optimal du contrôle interne du service public, je partage l'approche de Werner Schwaderlapp. Je rappellerai toutefois qu'une forme de contrôle externe existe, par le biais de la Commission d'évaluation des besoins de financement des établissements publics de l'audiovisuel (Kommission zur Ermittlung des Finanzbedarfs der Rundfunkanstalten, $K E F$ ). La KEF joue un rôle primordial pour la fixation du montant de la redevance. Elle est composée d'experts et contribue à un contrôle effectif. Mais elle doit fonder son évaluation sur la définition existante des missions du service public et ne peut pas en modifier le périmètre. Il est primordial pour l'audiovisuel public qu'il réfléchisse de manière plus poussée à sa propre mission au service de la société. S'il n'y parvient pas en interne, alors je pense comme Werner Schwaderlapp qu'il faut confier cette tâche à un conseil d'experts externes.

\section{Ne pas confondre concentration et capacité d'influence}

J. Rigaud (dans la salle) : J'avais souhaité dire un mot à propos de Berlusconi. J'ai été très satisfait d'entendre le Professeur Dörr défendre de manière aussi catégorique le principe selon lequel un étranger européen a les mêmes droits qu'un national dans l'attribution des chaînes.

Je voudrais donner un témoignage. J'ai été non seulement président de RTL mais aussi administrateur délégué de ce qui s'appelait la Compagnie Luxembourgeoise de Télédiffusion, devenue RTL Group dans les années 1990. Bertelsmann n’est devenu l'actionnaire unique de RTL Group qu'après l'an 2000. Je n'ai pas vécu cette période. Mais j'ai vécu d'abord, depuis 1983, la présence de Bertelsmann au capital de la CLT et, à partir de 1995, la parité entre la CLT et UFA, qui était la filiale audiovisuelle de Bertelsmann.

Ce que je sais du fonctionnement de RTL Group depuis l'an 2000 me confirme dans ce que je vais vous dire, à savoir que le Groupe Bertelsmann est suffisamment intelligent pour avoir compris que l'audiovisuel français est affreusement compliqué, très politique, et que moins il s'en mêle directement, mieux il sert ses intérêts. Le groupe Bertelsmann a une vision très claire de la rentabilité du groupe et des règles de management et il s'est bien gardé de confier à d'autres qu'à des Français la gestion des antennes françaises, que ce soit en radio ou en télévision. C'est la philosophie-même du groupe luxembourgeois en Belgique, en Hollande, en Allemagne et dans les autres pays. Ce sont les mêmes nationaux du pays d'intervention qui ont la responsabilité des programmes, des contenus, et avec une liberté d'initiative et un fonctionnement interne qui garantit le caractère européen de cette activité. Je signale cela en toute indépendance, puisque je ne suis plus rien dans ce groupe mais je voulais donner ce témoignage pour bien montrer combien le cas de Berlusconi est un cas tout à fait exceptionnel, et je dirais même du point de vue de la démocratie, pathologique. Il n'est plus président du Conseil en Italie, mais il peut le redevenir.

Jean-Louis Missika (dans la salle) : Je voudrais revenir sur la question de la fusion entre CanalSat et TPS en France parce que ce cas illustre bien l'écart qui peut exister 
entre le droit et ce qu'on pourrait appeler le climat d'opinion ou la capacité d'influence. Il me semble qu'en droit pur, on est face à la création d'un monopole. C'est une plateforme de télévision payante qui va se situer en position monopolistique pendant au moins trois ou quatre ans, le temps que d'autres opérateurs ou d'autres acteurs venus des télécommunications ou de l'accès Internet puissent concurrencer cette offre de services.

D'ailleurs, ce qui est assez intéressant, c'est qu'il est possible que cette décision du Conseil de la Concurrence et du ministère de l'Economie et des Finances précipite l'entrée des opérateurs de télécommunications sur le marché des contenus parce qu'en créant des conditions de position dominante pour les plateformes de télévision payante, ils incitent les autres à rentrer sur ce marché. Ce serait dans ce cas une sorte de prophétie auto-réalisatrice.

Mais je voudrais expliquer pourquoi, dans un pays aussi agité que la France, cette fusion et cette création d'un monopole n'ont posé aucun problème. Cela n'a posé aucun problème parce qu'il y a une véritable capacité d'influence des groupes concernés : TFI, M6, Lagardère, Canal+, constituent la quasi-totalité des médias français, en tout cas les plus puissants d'entre eux. On a coutume de dire, sur le mode ironique bien sûr, que l'Assemblée Nationale se divise en deux groupes parlementaires : le groupe TF1 et le groupe Canal+. Quand TF1 et Canal+ sont capables de porter une proposition politique unitaire, il est clair que leur capacité d'influence est extrêmement importante. Je ne dis pas qu'il n'y a pas eu une tentative de la part du Conseil de la Concurrence de résister à ce processus. Plusieurs de ses membres ont essayé de faire entendre un autre point de vue. Je pense notamment à la question de l'intégration verticale et de la question de Studio Canal, la structure qui chez Canal+ contrôle les droits des films et finance le cinéma français. Pour finir, même la possibilité de séparer Studio Canal du reste de l'activité de télévision payante n'a pas été retenue par le Ministère de l'Economie et des Finances.

Dernier élément, la comparaison avec l'Italie et l'Espagne me semble tout à fait contestable parce qu'en Italie et en Espagne, la seconde plateforme de télévision payante était au bord de la faillite. Elle allait fermer, ce qui n'était pas le cas de TPS en France.

Ce qui me paraît vraiment intéressant dans la fusion TPS CanalSat, c'est qu'elle est considérée aujourd'hui par l'ensemble des professionnels français et de la classe politique française plutôt comme une bonne chose, alors qu'elle crée, même si c'est de façon limitée dans le temps, un monopole de fait.

\section{Une nouvelle garantie du pluralisme adaptée à l'ère du numérique ?}

E. Flüry-Hérard : Je voudrais apporter quelques précisions à propos de ce dossier. Le monopole en question n'est pas un monopole sur la télévision payante, c'est un monopole sur la télévision payante par satellite. Il y a la télévision payante par ADSL qui croît de façon très rapide et la télévision payante sur le câble qui subsiste.

Deuxième remarque : quand le CSA a donné son avis au Conseil de la Concurrence, il a longuement insisté sur l'aspect intégration verticale et donc sur l'aspect acquisition des droits de diffusion de cinéma, de programmes audiovisuels, et la production de programmes audiovisuels et de cinéma. Ce qui est intéressant, c'est que le Conseil de la Concurrence n'a pas du tout repris ces analyses du CSA mais a considéré que l'opération de fusion ne changeait pas les positions de la nouvelle entité dans le domaine de la 
production cinématographique, ce qui est un raisonnement parfaitement normal en droit de la concurrence. Mais on voit bien que, du point de vue du pluralisme où se situait le CSA, le problème existait, même si ce n'était pas un problème du droit de la concurrence.

J.-L. Missika : Je suis d'accord avec cette analyse. Je pense qu'effectivement, il y avait un écart entre la position du CSA et la position du Conseil de la Concurrence. Simplement, quand vous dites que le monopole ne concerne que la télévision payante par satellite et que les services sur l'ADSL et sur le câble sont de nature différente, je pense que cette position est très théorique.

En réalité, un gros problème aujourd'hui est de savoir quel est le marché pertinent. Est-ce qu'on considère qu'il y a un marché de télévision payante par satellite différent du marché de la télévision payante sur le câble et l'ADSL ? Ou alors est-ce qu'on considère qu'il y a un marché de la télévision payante ? Si on considère qu'il y a un marché de la télévision payante, on constate qu'aujourd'hui les opérateurs de l'ADSL, France Télécom, Neuf Télécom et Free, sont obligés de vendre un paquet qui s'appelle CanalSat. Et si ce n'est pas le cas sur le câble, c'est qu'il y a une antériorité du câble par rapport à CanalSat. Mais demain, CanalSat proposera aux câblo-distributeurs de vendre un paquet qui s'appellera CanalSat.

Isabelle Bourgeois (dans la salle) : Je souhaiterais poser une dernière question à D. Dörr. C'est le Tribunal constitutionnel qui a défini le principe de garantie du pluralisme et ses implications. Or il a toujours adapté sa philosophie du droit aux évolutions de la société, révisant parfois sa propre doctrine jusqu'au changement de paradigme. Ce sont les juges suprêmes qui ont conçu, en 1986, "l'ordre dual de la radiodiffusion », le mode allemand de coexistence du public et du privé, et dans lequel le service public n'a une place privilégiée et une mission fondamentale que tant que le secteur privé n'est pas en mesure d'assurer un service de qualité équivalente. Ce double régime vise lui aussi à garantir le pluralisme.

Mais le Tribunal constitutionnel l'a lui-même présenté comme transitoire, estimant que des garanties particulières ne s'imposeront plus lorsque l'offre audiovisuelle sera suffisamment conséquente, et que la concurrence des multiples contenus suffira, comme dans le domaine de la presse écrite, à garantir le pluralisme. Les mutations des médias ont démultiplié l'offre. N'est-il pas concevable que la Cour modifie maintenant son approche de la garantie du pluralisme ?

D. Dörr : Bien sûr que cela est possible. Le Tribunal constitutionnel fédéral a toujours tenu compte des évolutions du contexte pour y adapter sa jurisprudence relative aux médias, mais toujours progressivement. Le pivot en était à l'origine la pénurie de fréquences. Depuis, les supports se sont démultipliés, et un marché médiatique s'est constitué. Il en tient compte dans sa nouvelle jurisprudence, tout en restant fidèle à la garantie fondamentale du pluralisme. Cela fut le cas par exemple entre son $3^{\mathrm{e}}$ et son $4^{\mathrm{e}}$ arrêt sur la radiodiffusion : en 1981, il soumettait encore le secteur privé à naître aux obligations du service public en matière de pluralisme ; ces règles ont été allégées en 1986, ce qui a laissé une grande marge de manœuvre aux chaînes privées allemandes qui naissaient à cette époque.

La réglementation allemande de la garantie du pluralisme est plutôt généreuse en comparaison européenne. Cela n'est possible que parce que le dualisme public/privé 
repose sur un service public fort dont l'existence légitime les activités d'un secteur privé aux missions réduites. La complémentarité entre ces deux types d'acteurs contribue ainsi au pluralisme sur l'ensemble du marché audiovisuel, donc au bon fonctionnement de la démocratie.

Or à l'heure actuelle, une garantie du pluralisme est plus nécessaire que jamais. Car contrairement à ce qu' on pensait, le nouveau monde médiatique, le nouveau 'kiosque électronique', n'a pas abouti à moins mais à plus de concentration, comme le révèle notamment la KEK dans ses rapports sur l'état de la concentration des médias. De nouvelles perspectives surgissent. Les plateformes offrent de nouvelles opportunités, la concurrence dans ce segment présente de nouvelles chances, de même que le rapprochement entre opérateurs de réseaux et de contenu.

Le Tribunal constitutionnel fédéral fera bien évidemment évoluer sa jurisprudence en conséquence, mais prudemment, et sans jamais perdre de vue la garantie du pluralisme qui est au fondement de la démocratie. Et le législateur, lorsqu'il donnera forme concrètement aux principes du droit fixés par la Cour suprême, sera amené lui aussi à réviser sa réglementation en matière de pluralisme. Il dispose pour cela d'une certaine marge de manœuvre. Il n'est par exemple nullement tenu de s'inspirer du modèle de la KEK. Ce qui importe, c'est qu'il adopte des règles efficientes. Et, comme Victor Henle, j'estime qu'il faut simplifier et moderniser le système allemand. Cette tâche revient au législateur. 



\section{Debat $\mathbf{N}^{\circ} 3$}

PARTS DE CAPITAL OU PARTS D'AUDIENCE.

Ces critères sont-ils encore adaptés à l'ère du numérique? 



\title{
DEBAT $\mathbf{N}^{\circ} 3$
}

\section{PARTS DE CAPITAL OU PARTS D'AUDIENCE. Ces critères sont-ils encore adaptés à l'ère du numérique?}

\author{
EXPOSE INTRODUCTIF : Isabell Hülsen (Financial Times Deutschland) \\ INTERVENANTS : \\ Alain Lancelot (Commission sur les problèmes de concentration dans le \\ domaine des médias) \\ Jürgen Heinrich (Université de Dortmund) \\ Dieter Schmidtchen (Université de Sarre) \\ Débat animé par Isabelle Bourgeois (CIRAC)
}

\begin{abstract}
Les critères retenus pour le contrôle des concentrations ou la garantie du pluralisme dans l'audiovisuel diffèrent dans nos deux pays : capital en France (pluralisme interne : au moins trois actionnaires au tour de table des opérateurs), parts d'audience outre-Rhin (contrôle externe). Mais à l'ère du numérique, le périmètre de l'audiovisuel est devenu flou, et ces deux critères ont perdu leur pertinence.

L'état de la réflexion sur la modernisation de la réglementation diffère lui aussi des deux côtés du Rhin. En France, l'interventionnisme étatique se conjugue à une méfiance intrinsèque vis-àvis des réalités du marché. La priorité est dès lors de développer, dans les milieux scientifiques et opérationnels chargés de l'évaluation de la concentration du secteur, la connaissance des mécanismes du marché médiatique. Ce n'est que sur cette base que pourra ensuite être abordée la question du pluralisme des opinions - une question qui, de surcroît, n'a qu'un lien très éloigné avec un droit de la concurrence de création par ailleurs très récente.

En Allemagne, où règne le modèle du libéralisme organisé et où le droit de la concurrence est une réalité ancienne, la priorité est à l'évaluation de l'impact économique de la révolution technologique et d'un regain de concurrence. L'approche actuelle de la régulation est frappée d'obsolescence, d'autant que la question du pluralisme s'est déplacée du niveau de l'offre vers celui de la demande. Et à l'avenir, il s'agira de se concentrer sur le rôle que jouent les médias classiques, linéaires, dans la socialisation des citoyens et la stabilité du lien social.

Or à l'inverse de la France où, selon le rapport de la Commission Lancelot, la concentration n'a pas atteint un degré alarmant, le marché allemand de l'audiovisuel est hautement concentré au niveau des opérateurs. Cela est dû paradoxalement à une régulation qui conférait une position prédominante à l'audiovisuel public tant dans le hertzien terrestre que sur le câble, où sa situation quasi-monopolistique contribue à entretenir une pénurie des vecteurs de distribution et à handicaper la concurrence...
\end{abstract}





\title{
EXPOSE INTRODUCTIF
}

\section{De l'incompatibilité entre parts de marché et parts d'audience}

\author{
Isabell Hülsen
}

Pourquoi les modèles de régulation font-ils actuellement l'objet de débats en France comme en Allemagne ? Je vais tenter d'en rassembler ici les principales raisons - du point de vue de l'observateur des réalités qu'est tout journaliste.

Du côté allemand, une polémique est née du projet de rachat, par les éditions Springer, de ProSiebenSat.1, deuxième groupe de télévision en Allemagne. Ce projet, qui n'a pas abouti, a suscité de vives discussions sur la pertinence de la régulation, de ses institutions, face à l'interpénétration croissante des différents médias. Celles-ci se sont concentrées sur le cœur du dispositif : le $\S 26$ du Contrat d'Etat sur la radiodiffusion (Rundfunkstaatsvertrag) conclu par les Länder et qui a force de loi sur l'ensemble du territoire.

Un autre cas avait précédemment révélé la nécessité de réviser la régulation. Dans le cadre de la création, en décembre 2005, de la chaîne payante Arena consacrée au football, un câblo-opérateur, Unity Media, avait obtenu une licence. L'obtention était certes indirecte, mais elle a créé un précédent. La tendance à l'intégration verticale des médias, c'est-à-dire à la fusion entre réseau et contenu (elle est beaucoup plus répandue en France qu'en Allemagne) est ainsi brusquement devenue palpable.

Or les opérateurs de plateformes comme les câblo-opérateurs et les opérateurs satellites, dont Astra et son projet de plateforme satellite numérique, investissent de plus en plus le segment des contenus. Mais s'ils ont à respecter les règles du libre accès, ils échappent à celles de la garantie du pluralisme d'opinions. S'ajoutent à la liste des nouveaux acteurs de l'audiovisuel les groupes de télécommunications. Deutsche Telekom, utilisant lui aussi le football comme produit d'appel, a lancé à l'automne $2006 T$ Home, sa télévision sur Internet, avec plus de 100 chaînes. La multiplication des plateformes remet en question le rôle de référence qui revenait aux chaînes de télévision.

Quant à la progression mondiale de la télévision par Internet, on peut la considérer sous deux angles: soit simplement comme la diffusion de ce média classique sur un nouveau support soit comme l'incarnation par excellence de la convergence entre «tuyaux et contenus ». Car cette télévision en ligne ne se contente pas de dupliquer l'offre traditionnelle, elle propose aussi à un large public films, shows et autres prestations à la demande qui n'obéissent pas au schéma établi des programmes linéaires. D'où la distinction effectuée par la Commission européenne entre communication linéaire et non linéaire. C'est dans cette dernière catégorie qu'entrent aussi des sites comme You Tube ou My Space : ils ne proposent plus seulement une offre de contenus générés par les utilisateurs mais ils ont tendance à devenir des plateformes commerciales pour différents contenus médiatiques dans la mesure où ils agissent en collaboration avec des 
moteurs de recherche comme Google ou avec des maisons de disques qui utilisent les pages du site comme des supports de clips vidéo.

Internet est également en train de mettre à mal le rôle central de gate keeper qu'avait la télévision : ce ne sont plus les grilles de RTL, ProSieben ou N24 qui vont guider nos choix de consommation médiatique, mais notre consultation de sites comme www. spiegel.de ou www.yahoo.com, une recherche sur Google, le téléchargement d'un épisode de série télévisée sur I-Tunes ou des portails comme Intomovies ou encore Maxdome...

\section{Le droit allemand est mal armé pour prendre en compte l'intégration pluri-médias}

Toutes ces évolutions ont avivé les débats sur la garantie du pluralisme ou le contrôle des concentrations. Deux tendances apparaissent comme des défis majeurs : l'interpénétration des médias s'accroît, et la télévision traditionnelle perd de son influence sur le marché pluri-médias. Par ailleurs, au sein du segment de la télévision, l'influence des chaînes en tant que diffuseurs d'une offre composée, linéaire, diminue au profit de nouveaux acteurs : opérateurs de réseaux, de plateformes ou navigateurs de programmes.

Le consommateur de médias est confronté à une abondance nouvelle de l'offre qui lui permet d'orienter ses choix d'une manière plus autonome. Certes, quantité n'est pas nécessairement synonyme de pluralisme. Mais la grande diversité de l'offre révèle à quel point est inadaptée aujourd'hui un système de régulation centré sur les seuls opérateurs établis de radiodiffusion et qui ne s'intéresse aux stratégies pluri-médias - plus en Allemagne, il est vrai, qu'en France - que lorsqu'un opérateur s'est taillé la part du lion sur le segment traditionnel de la télévision.

Pour le droit allemand, une combinaison de type moteur de recherche + portail Internet + quotidien à fort tirage Bild ne représente en rien un danger pour la formation de l'opinion - ce présupposé pourrait d'ailleurs se révéler exact, mais comme personne ne s'est jamais penché sur la question, aucune conclusion n'est possible. Le droit français des médias prend certes mieux en compte la diversification multimédias, dans la mesure où il limite a priori les parts de capital sur les différents segments du marché des médias. Mais il n'en reste pas moins lui aussi centré sur la télévision en tant que référence.

Le système allemand de régulation est bien mal armé pour affronter les mutations des médias. Le projet de fusion avorté entre les groupes Springer et ProSiebenSat.1 le révèle de manière exemplaire.

Conformément à l'art. 5 \$1,2 de la Loi fondamentale, le législateur doit prendre des dispositions de nature à prévenir l'émergence d'un opinion dominante. Cette mission est concrétisée notamment par l'art. 26 du Contrat d'Etat sur la radiodiffusion (Rundfunkstaatsvertrag), conclu par les Länder et qui a force de loi sur l'ensemble du territoire. Du fait de sa large diffusion, la télévision est apparue comme le média exerçant potentiellement la plus grande influence sur l'opinion et représentant ainsi le plus grand danger potentiel pour le pluralisme.

Si cette approche est la même qu'en France, les seuils et critères retenus pour prévenir l'émergence d'une «opinion dominante » diffèrent : alors que la réglementation française limite les parts que peut détenir une société au capital d'un opérateur (dès lors que son audience dépasse $2,5 \%$ ), le droit allemand prend en considération la part d'audience réalisée par l'ensemble des chaînes d'une société de programmes. 


\section{La télévision reste la référence pour mesurer une « position d'opinion dominante »}

Selon cet art. 26 du Contrat d'Etat de la radiodiffusion, il y a présomption de «position d'opinion dominante » dans trois cas. Dans le premier, les chaînes d'une société de programmes cumulent une part d'audience de $30 \%$ sur le seul segment de la télévision. Dans le second, cette part est réduite à $25 \%$ lorsque la société est en position dominante sur un "marché voisin médiatiquement pertinent ». Enfin, troisième cas : les parts d'audience cumulées de la société à la fois sur le segment de la télévision et sur les «marchés voisins médiatiquement pertinents » s'élèvent à l'équivalent de $30 \%$ de parts d'audience télévisée.

Par ce concept de "marchés voisins médiatiquement pertinents » qui s'applique à la radio, la presse ou Internet, le législateur cherche à prendre en considération la tendance à l'interpénétration des médias. Mais les nombreuses études et expertises consacrés à ce sujet et l'échec du projet de fusion Springer/ProSiebenSat.1 en application du troisième cas prévu à l'art. 26 révèlent les faiblesses inhérentes à ce concept, de plus en plus patentes au fur et à mesure que se concrétisent les tendances à la convergence.

Certes, la loi tient compte du rayonnement d'une chaîne au-delà du segment de la télévision. Mais le concept créé à cet effet : ces "marchés voisins médiatiquement pertinents », est tout sauf convaincant et même consistant. Car il implique d'une part qu'on établisse une grille mesurant l'importance comparée de la presse écrite ou des médias en ligne et de la télévision et, d'autre part, qu'on dispose d'outils aussi fiables que la mesure de l'audience télévisée pour évaluer les parts de marché de la société de programmes en question sur les segments de la presse, de la radio, de l'offre en ligne. Enfin, ce concept présuppose l'adoption d'un barème pour convertir ces parts de 'marché' partielles et respectives en termes de parts d'audience télévisée ou d'influence possible sur l'opinion.

La Commission de l'évaluation de la concentration dans les médias (la KEK), qui a pour mission d'appliquer cet art. 26, a eu dans le cas du projet de fusion Springer / ProSiebenSat.1 la tâche ingrate de devoir définir pour la première fois le poids relatif des autres médias face à la télévision et de devoir appliquer cette évaluation à un cas délicat et épineux : elle utilisa pour l'évaluation les critères d'actualité, de force suggestive, et d'accessibilité à la masse énoncés par le droit constitutionnel. Elle parvint à la conclusion que le pouvoir d'influence des quotidiens sur l'opinion s'élève aux deux tiers de celui de la télévision. Comme la part de marché des titres du groupe Springer dans le segment de la presse est de $26 \%$, la KEK a estimé que, selon ce barème, elle équivalait à une part d'audience télévisée de $17 \%$. Elle a procédé de la même manière pour les périodiques et l'offre Internet du groupe.

Ce procédé a été vivement critiqué. Théoriquement, ce barème de conversion peut déboucher sur des 'parts d'audience' cumulées de plus de $100 \%$. Plus fondamentalement se pose la question de savoir comment délimiter ces marchés pertinents. Le tirage et même la diffusion d'un quotidien ne donnent aucune indication sur l'usage qu'en fait l'abonné ou l'acheteur - une donnée décisive pourtant, puisque l'utilisation du média est l'aune à laquelle se mesure l'audience TV, la référence en la matière. Et ainsi de suite...

Le flou du concept se traduit par de lourdes incertitudes juridiques pour toute entreprise qui est dès lors amenée à se demander s'il est pertinent d'investir des milliards alors que la réponse du régulateur à un dossier de candidature est imprévisible. 


\section{Appliquer le droit commun de la concurrence aux médias ou affronter la question de fond : quelle place pour la TV ?}

Et le problème deviendra insoluble lorsqu'il s'agira de tenir compte non seulement des parts dans les médias, mais aussi des 'parts d'audience' ou de l'influence sur l'opinion d'un opérateur de réseau proposant ses propres contenus ou bouquets de chaînes...

Le droit ne considère en effet pas ce cas de figure sous l'angle du consommateur (garantie du pluralisme), mais simplement sous celui de l'offre. L'art. 53 du Contrat d'Etat sur la radiodiffusion se contente de garantir le libre accès au réseau à tout offreur de programmes. En l'état actuel du droit, il faudrait que Unity Media se rapproche de ProSiebenSat.1 et de quelques autres encore pour que l'opérateur fasse l'objet du contrôle des concentrations propre aux médias. Ce n'est donc pas sans raison que même des adversaires invétérés de l'idée même d'une régulation comme Georg Kofler, président de Premiere, réclament soudainement une régulation ; c'est pour mieux asseoir leur position face à la montée en puissance des opérateurs de réseau.

Il n'y a que deux solutions pour adapter la régulation à la convergence. La plus radicale serait de la confier à l'Office fédéral des Cartels. Cela impliquerait d'abandonner la fixation sur un seul média, la télévision, au profit de la prise en considération de tous les médias ; le marché de référence serait alors multimédias. La seconde est plus pragmatique. Elle consiste à faire évoluer les règles existantes pour définir des lignes directrices et des modes d'appréciation plus opérants. Comme cette approche élude la question de fond, certains y voient une solution de facilité. En un mot, le débat est ouvert... 


\section{Discussion}

Isabelle Bourgeois : Le Premier ministre Jean-Pierre Raffarin avait institué une Commission chargée d'évaluer «les questions de concentration dans les médias ». Présidée par Alain Lancelot, elle a rendu son rapport en décembre 2005, sous le Premier ministre Dominique de Villepin. Dans ce document fondamental pour la compréhension des problématiques économiques et constitutionnelles liées à la réglementation des médias en France, la commission parvient à une conclusion qui a profondément choqué les observateurs allemands puisqu'elle affirme en substance que le marché français n'a pas atteint un degré de concentration alarmant. Comment s'explique ce constat?

Alain Lancelot: J'ai le sentiment, après le début de notre rencontre, qu'il existe dans nos deux pays, qui sont pourtant très semblables et qui coopèrent depuis plus de 50 ans dans l'Union Européenne, des attitudes profondément différentes dans le domaine des médias qui traduisent de grandes différences culturelles: différence de cultures politico-juridiques d'une part et différence de cultures économiques d'autre part.

De profondes divergences en matière de cultures politiques et économiques

Considérons d'abord les divergences de culture politique.

La République française s'est développée sous le signe du rassemblement et de l'unité, notamment l'unité de l'intérêt général qui ne peut pas être divisé, alors que la République fédérale s'est construite sous le signe de la diversité et du respect de la subsidiarité. On y considère qu'il doit y avoir des niveaux variés dans la définition de l'intérêt général, une sorte d'échelle où l'on se gouverne librement à chaque échelon en dehors du 'grand tout' normatif que l'Etat-Nation impose à la culture française. Voilà pour la première différence, fondamentale, dans le domaine des médias.

Deuxième différence: si l'Etat a un sens en France, c'est le pouvoir suprême détenteur de fait de la légitimité et qui tombe d'en haut, c'est le règne du commandement, alors qu'en Allemagne, il signifie d'abord la société civile. Cela est apparu au lendemain de 1945, où l'on a vu l'occupant affirmer sa volonté de ne plus jamais voir "das Reich », mais de voir au contraire dans la diversité de la société civile les moyens d'ancrer une démocratie vivante. Alors que nous avons en France la verticalité de la décision souveraine, nous avons en Allemagne l'horizontalité du contrat, les relations de pair à pair.

Une troisième différence vient de ce que nous avons, du côté français, une tradition d'interventionnisme, qui fait que dans tous les domaines, les pouvoirs publics doivent être présents et si possible les derniers à avoir la parole. En revanche, dès qu'il s'agit de réguler, nous nous trouvons en face de cultures qui mettent en jeu des modèles profondément étrangers à la culture politique nationale.

La différence politico-économique est encore plus préoccupante que la différence politico-juridique. En France, l'économie de marché est encore suspecte alors qu'en Allemagne, elle est depuis longtemps légitime. Si bien que dans le monde des médias, 
l'économie est un véritable trou noir. En grossissant à peine, on pourrait résumer le préjugé antiéconomique par quelques slogans, comme "le capital, voilà l'ennemi », "l'information n'a pas de prix » ou "le journal n'a pas de prix ». La seule valeur qui compte, c'est l'indépendance, voire la liberté illimitée, des journalistes. L'idée que, pour qu'il y ait des journalistes, il faut qu'il y ait des journaux, et que pour qu'il y ait des journaux, il faut qu'il y ait des gens qui les achètent, relève de "l'horreur économique ».

Que peut faire la puissance publique si elle ne se nourrit pas des nos idées qui sont nécessaires à la démocratie ? A quoi sert-elle si elle ne paye pas les journalistes pour donner du sens à sa politique ? Ceci peut paraître caricatural, mais en temps que viceprésident du Centre de Formation des Journalistes pendant dix ans, je peux vous garantir que chaque fois que j'ai rencontré les représentants des syndicats ou même les jeunes étudiants en journalisme, j'ai été sidéré de voir qu'ils ne pouvaient ni admettre ni même comprendre que leur métier devait obligatoirement s'inscrire dans le cadre d'une industrie.

La concentration au niveau des entreprises n'est pas nécessairement dommageable au pluralisme des opinions

Dans la commission que j'ai eu l'honneur de présider, et à laquelle Francis Balle a apporté toute sa science et tout son engagement, nous avons cherché, puisqu'on nous avait demandé de travailler sur la concentration dans les médias, de savoir quel était en France le degré de concentration, et il nous est très vite apparu que, par rapport à la situation des pays comparables, la situation en France était loin d'être alarmante.

Car nous n'avons pas de grands groupes de presse, hélas, et notre presse qui ne cesse de se détériorer. En 20 ans, la presse écrite a perdu $20 \%$ de sa diffusion. Aujourd'hui, en 2006, on compte en moyenne 181 exemplaires de la presse écrite diffusés pour 1000 habitants en France, contre 371 en Allemagne, 540 en Suède, et je ne parle pas du Japon. Pourquoi ? Parce la presse française n'est pas rentable. Pourquoi n'est-elle pas rentable ? Parce qu'elle ne comprend pas les problèmes de rentabilité. Il n'en est pas question dans la production, liée à un système de closed shop pour les syndiqués du «Livre » et à des pratiques protectionnistes chez les journalistes. Et il n'en est pas question dans la distribution : pas ou très peu d'abonnements, pas de portage, contrairement à ce qui se passe dans la plupart des pays où la presse est vivante, mais seulement la vente au détail beaucoup plus coûteuse.

Ces défauts structurels font de la presse un objet très cher. J'ai fait le calcul sur ce que me coûte personnellement la presse écrite : en achetant Le Monde tous les jours, je dépense 68,40€ par mois alors que je peux m'abonner à l'hebdomadaire Le Point pour $9 €$ par mois. Comme, de surcroît, j'habite en province et que j'achète La Nouvelle République du Centre Ouest pour connaître l'actualité départementale et municipale de ma petite ville, cela fait 80 centimes de plus par jour. La presse française est chère pour ce qu'elle apporte, surtout en comparaison de la densité des informations fournie par un quotidien allemand, et à un prix inférieur.

Lorsque le Premier Ministre m'a reçu pour me demander de créer cette commission, sa préoccupation était nourrie par l'actualité de la presse : le groupe Dassault s'apprêtait à vendre trois petits journaux de province (dans les départements de la Mayenne, du 
Maine et Loire et de Loire-Atlantique) qui risquaient de tomber dans l'escarcelle du groupe Ouest France dont le principal titre du même nom a un tirage de 800000 exemplaires, le plus gros de tous les quotidiens généralistes en France, mais dans la moyenne de la plupart des journaux européens. Dassault voulait aussi entrer dans le capital de TF1, ce qui n'était pas sans soulever quelques difficultés en termes de concentration. Je lui ai dit alors que la première chose à faire était d'établir un diagnostic de l'économie des médias, en tenant compte non seulement de la presse et de la télévision, mais aussi d'Internet et des télécommunications. Il a paru plutôt surpris mais a fini par se ranger à mes arguments, et c'est ainsi que nous avons défini la mission de cette commission.

La difficulté de notre mission résidait dans l'articulation entre liberté de la concurrence et liberté, ou pluralisme, des opinions. Les entreprises de presse sont des entreprises, mais elles ne sont pas que cela puisque, pour le Tribunal constitutionnel allemand comme pour la règle française, elles doivent être soumises aussi à une autre forme de régulation qui concerne le respect du pluralisme des opinions qui donne un sens au choix démocratique.

Mais il ne faut pas confondre systématiquement la puissance économique des médias et la liberté d'opinion. On peut avoir un système où coexistent une forte concentration économique au niveau des entreprises et une grande concurrence au niveau des titres - souvent même édités par une seule société. Il y a de nombreux cas. Le maire de Marseille, Gaston Defferre, socialiste, a par exemple longtemps été le patron du journal socialiste et du journal réactionnaire de Marseille. Il en était le propriétaire et il faisait en sorte que les deux familles politiques de la ville de Marseille puissent avoir les messages qu'elles attendaient et il se réjouissait de pouvoir en tirer les bénéfices : le pluralisme était respecté, bien qu'il y eût derrière un seul groupe.

Nous nous sommes préoccupés dans la commission de savoir s'il fallait mesurer la concentration en termes de diffusion, comme c'est le cas pour la presse écrite, ou de capital comme c'est aujourd'hui le cas dans l'audiovisuel.

Dans la presse écrite, le critère est un seuil d'audience : aucune entreprise de presse ou groupe de presse ne peut disposer de plus de $30 \%$ de la diffusion dans la presse écrite. Dans l'audiovisuel, et surtout la télévision, qui est aujourd'hui encore le média le plus sensible, le critère est capitalistique, aucun actionnaire ne devant dépasser le seuil de $49 \%$ du capital. Il nous est apparu que cette mesure n'était ni très fiable ni très efficiente, comme nous l'avons entendu dire tout à l'heure à propos de l'Allemagne, et qu'elle posait beaucoup de problèmes. Pour détenir le contrôle d'une société, il suffit en fait de contrôler $35 \%$ ou $40 \%$ du capital. De plus, lorsqu'il s'agit de sociétés cotées en bourse, il est extrêmement difficile d'avoir un capital fixe. Les capitaux sont si mobiles qu'il est très difficile de savoir à quel moment on dépasse ou non le seuil indiqué.

Il nous est apparu alors qu'il était plus logique, puisqu'il s'agissait moins de contrôler la concentration capitalistique que d'assurer la défense du pluralisme, il nous a paru plus juste de considérer ce qui touche le plus le pluralisme, c'est à dire l'audience. Quelle est l'influence que chacun des consommateurs de presse peut recevoir? Risquent-ils ou non d'être 'bombardés' par une diffusion à sens unique ? La France a bien connu cela du temps où l'Etat était le seul responsable de son système audiovisuel. C'était le bombardement constant d'informations par un audiovisuel qui, pour reprendre le mot de Georges Pompidou, était la «voix de la France »... 
I. Bourgeois : Alain Lancelot a évoqué la profonde méfiance qui règne en France envers le capital en général. En Allemagne, et malgré les différences culturelles décrites, cela semble être le cas aussi dans le domaine de l'audiovisuel. Je voudrais citer un bref constat tiré du rapport réalisé en 1999 par le Conseil d'évaluation scientifique placé auprès du ministère fédéral de l'Economie et de la Technologie et qui préconisait une libéralisation du système allemand de régulation des médias. Le constat est le suivant : "Le droit actuel jette le bébé avec l'eau du bain. Il est marqué par une profonde méfiance envers l'apport positif de la concurrence. Le législateur ne fait pas confiance à l'audiovisuel privé ». Par-delà toutes les différences, voilà un point qui unit la France et l'Allemagne. Et pourtant, le marché allemand des médias présente un très fort degré de concentration...

Jürgen Heinrich : Cette méfiance n'est pas totalement infondée. Et il existe bien un parallèle entre nos deux cultures : il m'a fallu 25 ans pour faire comprendre à mes étudiants en journalisme qu'il existe un marché et des contraintes...

Je souhaiterais commencer par une remarque : j'interviens ici en tant qu'économiste. En tant que tel, je ne dois pas, contrairement à la KEK que préside D. Dörr, m'en tenir exclusivement au droit constitutionnel. En ce qui concerne le marché de l'opinion, les économistes ont un point de vue totalement différent de celui du Tribunal constitutionnel fédéral.

\section{Il est impossible de réguler la demande}

Quels sont les défis de la numérisation? Dans l'approche économique, elle signifie une intensification de la concurrence entre les réseaux ; la conséquence pour la régulation est que celle-ci n'a (presque) plus besoin de s'effectuer au niveau de chacun des réseaux pris isolément. La concurrence change de caractère : à la forte compétition au niveau des prix sur le marché s'ajoute la concurrence au niveau de l'innovation pour entrer ou se maintenir sur le marché. Globalement, la concurrence s'en trouve avivée. Mais il y a un problème : dans ce nouveau contexte, les acteurs développent des formes de coopération inédites, par exemple en constituant un réseau de traitement de l'information fédérant les sociétés de production audiovisuelle - autrement dit, une entreprise virtuelle. La structure de la concentration gagne en complexité et, dans la mesure où la concurrence s'intensifie, se multiplient les tentatives pour s'y soustraire.

Les conséquences sur le marché de l'opinion sont moins simples à décrire. Les travaux des spécialistes des sciences de la communication semblent révéler une nette distribution des modes de consommation selon les avantages comparatifs des différents médias : la presse est lue pour l'information locale qu'elle apporte, la radio et la télévision choisies pour le divertissement, et Internet pour ses fonctions d'orientation et de service.

Ensuite, l'offre gagne en diversité. Ce point est amplement thématisé. Il faut cependant ajouter que, d'un point de vue économique, la notion de pluralisme a deux facettes : elle relève de la problématique de l'offre et, plus encore, de celle de la demande. Or il est pratiquement impossible de réguler la demande de médias. La raison en est simple : le temps consacré par un citoyen éclairé à la collecte et au traitement de l'information lui interdit de profiter pleinement de l'offre dans toute sa diversité. Il ne peut pas tout consommer; nous appelons cela l'ignorance rationnelle. Et ce citoyen continuera 
de ce fait à rechercher une offre non seulement structurée, mais également identifiée par une marque, par exemple RTL2, Google ou Deutsche Telekom. Face à cette loi humaine de l'ignorance rationnelle, la politique des médias est condamnée à l'impuissance.

Autre point primordial : l'approche économique, celle des sciences de la communication, mais aussi tout simplement le bon sens voudraient qu'on aborde la notion de "marché de l'opinion» dans une perspective beaucoup plus large. Car il importe peu de savoir par le biais de quel média est diffusée une opinion, elle l'est, tout simplement. Il est dès lors illusoire de chercher à établir une hiérarchie des divers médias selon un hypothétique degré d'influence sur le processus de formation de l'opinion publique, ainsi que le proposent la KEK ou le professeur Uwe Hasebrinck de l'Institut Hans-Bredow à Hambourg.

Par ailleurs, contrairement à l'idée que s'en font généralement les constitutionnalistes, le champ de l'opinion ne se limite pas au pluralisme politique dans l'audiovisuel. Le pluralisme est un mécanisme de socialisation, d'intégration de l'individu dans la société, il détermine notre culture. Chacun des supports médiatiques y contribue, quel qu'il soit. C'est ce qu'enseignent les sciences de la communication.

En ce qui concerne la régulation, j'estime qu'il faut clairement distinguer deux aspects : d'un côté la nécessité d'une protection contre les monopoles ou positions dominantes sur le marché de l'opinion, et de l'autre la question de savoir comment préserver la haute mission de l'audiovisuel et, plus généralement, celle qu'ont tous les médias. Les économistes se demanderaient en priorité, comme vient de le faire Bernd Holznagel, si le marché est en mesure de mettre ces biens à disposition en quantité et qualité suffisantes. Nous constatons qu'il n'y parvient que partiellement. Or il s'agit de biens publics, c'est-àdire de biens dont doivent bénéficier tous les citoyens sans avoir à payer individuellement un prix d'accès. Il revient donc à l'Etat de financer ces biens publics de façon adéquate, peu importe qu'il le fasse par le biais d'un impôt ou d'une redevance.

Comment réguler le marché ? Il est assurément plus aisé de mettre en œuvre un contrôle au niveau de la structure des acteurs qu'à celui des comportements de consommation, voire de performance de l'offre. Ces deux derniers points plaident contre un modèle établi sur les parts d'audience, d'autant que nos approches de la performance de l'offre sont incompatibles avec la pensée économique. Une régulation par une limitation des parts de capital est inefficiente du fait de la complexité du contrôle qu'elle induit, comme des innombrables possibilités de le contourner qui s'offrent aux acteurs. En Allemagne, ce modèle n'a pas empêché la constitution d'un duopole audiovisuel. Il n'y a donc qu'une solution : renoncer à ces deux critères au profit d'un contrôle des participations en cas de projet de rapprochement ou de fusion.

Cela présenterait l'avantage de mieux prendre en compte la tendance à l'intégration verticale et à la concentration multimédias - à condition de considérer le marché de l'opinion dans sa globalité. Etant donné qu'il n'y a aucune différence, pour le processus de formation de l'opinion, entre une entreprise qui produit ce qu'elle diffuse et une autre qui se contente de distribuer des contenus ou opinions, il serait nécessaire en revanche d'abaisser le seuil au-delà duquel une opinion est considérée comme en position dominante. On pourrait envisager une part de $10 \%$. Mais il revient aux responsables politiques de prendre ce genre de décision. 


\section{II revient aux acteurs publics de préserver le lien social}

Le problème le plus difficile à résoudre concerne la garantie de la mission de service public. J'entends par là la socialisation et la préservation de la culture. Les subventions, de même que les obligations de programmes telles que les prévoit la Directive TSF dans l'UE ou la réglementation de l'audiovisuel en France me semblent des moyens adaptés et pour lesquels je plaiderais même en ma qualité d'économiste.

Un service public de l'audiovisuel dont la programmation doit répondre à des critères précis est d'autant plus indispensable qu'il est par ailleurs impossible aujourd'hui de définir une mission globale pour les médias en général, et donc d'en contrôler le respect.

Mais si le secteur public doit être ainsi préservé, il est indispensable, du point de vue de la raison économique comme de la conformité avec le droit européen, d'en réduire le périmètre et de le réformer. Le point de vue des économistes sur cette question est simple à comprendre : préserver la culture (au sens large) et le lien social d'une société est une mission prioritaire ; or le marché ne peut remplir cette mission qu'en partie. Elle doit donc être financée par la collectivité et confiée à des acteurs publics.

Reste la question la plus épineuse : qui contrôlera, et comment, l'exécution des missions par le service public ? Cette tâche pourrait revenir aux Landesmedienanstalten.

I. Bourgeois : J'aimerais que vous précisiez le concept sur lequel les régulateurs des médias comme vous-même fondez votre approche : celui de «marché de l'opinion »...

Un intervenant (dans la salle) : ... les économistes ne sont pas habilités à prendre position sur un sujet qui relève de la compétence du philologue des médias ! Quoi qu'il en soit, l'opinion ne peut pas être considérée comme un marché au sens classique. Dans un marché au sens classique, en effet, on paie pour obtenir quelque chose en échange. Cette transaction n'existe pas sur un marché de l'opinion où chacun peut exprimer ou s'approprier une opinion sans contrepartie. C'est pourquoi je préfère parler de secteur de l'opinion. Et, comme Dieter Dörr, j'estime que dans le secteur de l'opinion, aucun acteur ne doit avoir de position dominante - une position qui serait une vision d'horreur au demeurant pour tout économiste aussi. C'est pour cela que je rejoins Jürgen Heinrich lorsqu'il prône une définition large du secteur de l'opinion, mais avec une régulation reposant sur des seuils de concentration nettement plus faibles qu' aujourd'hui.

Dieter Schmidtchen : Dans l'ensemble, je suis d'accord avec mon collègue économiste. Il y a toutefois une divergence avec sa définition du marché de l'opinion. J'évite pour ma part de parler d'opinion ou de pluralisme d'opinions ; expression auxquelles je préfère celles d'information et de marché de l'information, dans la mesure où il y a offre et demande d'information (le fait de payer ou non cette information ne m'intéresse pas ici). Or l'information possède plusieurs dimensions qu'il s'agit de bien distinguer quand on évoque la question d'une délimitation du marché.

\section{« Marché de l'opinion » ou marché de l'information?}

La première dimension : la diffusion de nouvelles factuelles. En Allemagne, c'est le rôle des journaux télévisés des chaînes publiques : la première chaîne de l'ARD ou la ZDF. La deuxième repose sur le fait que, en Allemagne, on accorde beaucoup d'importance à la séparation entre les faits et le commentaire - c'est-à-dire de l'opinion du journaliste. Cette dernière est dès lors exprimée sous une forme particulière : l'éditorial, la tribune 
libre, l'analyse ou le «commentaire » déclaré (Kommentar). On la trouve en Allemagne dans la presse écrite comme dans l'audiovisuel. Cette catégorie constitue une deuxième dimension de l'information.

La troisième est celle de la consommation de l'information. Je souhaite me détendre le samedi soir en regardant un show télévisé. Je ne m'étendrai pas sur la question de savoir si les shows télévisés contribuent à entretenir le lien social, ce qui m'intéresse, c'est le fait que je consomme un show. L'information que je consomme ainsi ne détermine en rien un choix comme la décision d'acheter quelque chose. Si je regarde un show, c'est simplement pour le consommer, et rien d'autre. Mais je peux aussi choisir de consommer une information dans le but exprès d'y trouver des éléments me permettant d'effectuer des choix ou de prendre une décision, comme dans le cas d'une élection.

La quatrième dimension, enfin, concerne la socialisation, l'éducation. Les commentaires y contribuent, tout comme l'information factuelle, puisque des facteurs comme la sélection des faits et leur hiérarchisation permettent de guider le processus de formation de l'opinion.

Une fois cette distinction effectuée, j'en reviens à la question : qu'est-ce qu'un marché ? Je pourrais imaginer un marché en fonction de chacune de ces dimensions. Il faudrait alors que je les délimite. Dans la définition de l'Office fédéral des Cartels allemand, un marché se construit sur des besoins. Pour identifier ces besoins, on se demande quels biens - en l'occurrence quelles informations - peuvent ou pourraient se substituer à ceux qui sont disponibles. Les biens, les informations, qui sont facilement substituables, déterminent un marché dans cette définition orientée sur les besoins.

Dès lors, il faut prendre en considération les informations que mettent à notre disposition les différents médias. Quelles sont mes sources pour l'information factuelle ? La télévision, la radio, Internet, les quotidiens, une conversation avec mon partenaire lors d'une activité sportive commune, etc. Pour déterminer le marché de l'information dans sa dimension factuelle, je dois donc prendre en considération ces innombrables sources et biens. Et je devrais procéder de même pour délimiter un marché dans les trois autres dimensions de l'information... On voit bien la difficulté à délimiter le "marché ou secteur de l'opinion ».

I. Bourgeois : Et pourtant, en Allemagne, ce «marché de l'opinion » est la référence sur laquelle s'établit la régulation de l'audiovisuel. Il est vrai que ce modèle n'a été adopté par le législateur qu'après le constat d'échec du premier, construit, lui, sur une approche capitalistique comme c'est toujours le cas en France.

\section{Allemagne : effets pervers d'un contrôle des concentrations dans l'audiovisuel privé dont l'objet véritable était et est toujours de préserver la position centrale du service public}

Or certains observateurs, et non des moindres, estiment que les deux modes de régulation successifs sont la cause du haut niveau de concentration observé sur le marché de l'audiovisuel allemand. D'une part, parce que le seuil de $49 \%$ de parts de capital n'a nullement empêché ni l'intégration verticale ni la concentration horizontale multimédias. D'autre part, parce que le système établi aujourd'hui sur les parts d'audience ne s'applique qu'aux opérateurs privés de la télévision, c'est-à-dire à la moitié seulement d'un «marché de l'opinion » où les chaînes du secteur public cumulent quelque $50 \%$ 
des parts d'audience. Peut-on réellement imputer de tels effets pervers aux modes existants de contrôle du pluralisme ?

J. Heinrich : En effet, la régulation et, plus particulièrement le modèle visant à limiter les parts de capital au tour de table d'un opérateur, a favorisé la concentration sur le marché de la télévision depuis sa libéralisation dans les années 1980. Dans son rapport 2002/03, la Commission des monopoles, qui est le conseil scientifique de l'Office fédéral des Cartels, consacrait un chapitre à ses effets pervers, essentiellement la limitation de l'accès au marché de nouveaux concurrents.

Le Conseil scientifique auprès du ministère fédéral de l'Economie partage la même analyse ; l'un de ses membres est également le président de la Commission des monopoles. Les deux instances relèvent que le législateur est très sceptique quant à une régulation par le marché. Le législateur, de même que les juges de Karlsruhe, ont tendance à ne voir partout que défaillance du marché. Et ils estiment à juste titre que lorsque le marché est défaillant, l'Etat doit intervenir. Mais sous quelle forme ? Les Français se prononceraient pour la création d'entreprises publiques. Les Allemands ont choisi une voie similaire. D' autres encore estiment qu'il revient à l'Etat de réaliser cette prestation, par exemple en lançant des appels d'offres pour certaines missions, avec une dotation budgétaire afférente.

La méfiance envers l'apport positif de la concurrence est profondément ancrée en Allemagne. Et elle détermine les décisions des législateurs comme des juges constitutionnels. Cela a pour conséquence qu'une grande partie des mesures prises dans le domaine de la régulation sert à protéger le secteur public de la concurrence. Vu sous cet angle, la régulation s'est bel et bien traduite par une limitation de la concurrence. Cette thèse est elle aussi défendue par la Commission des monopoles.

I. Bourgeois : Vous faites allusion à cette "garantie de maintien et de développement» (Bestands- und Entwicklungsgarantie) du service public que les juges de la Cour de Karlsruhe avaient définie dans leur jurisprudence?

J. Heinrich : Il s'agit bien de la garantie de maintien de l'existant, de la garantie de développement et de la garantie de financement qui les accompagne. L'approche de la Cour est très généreuse ; elle implique que les chaînes publiques sont entièrement libres de définir leurs programmes et services comme elles l'entendent. En économie, on décrirait cela comme une illustration de la théorie du principal-agent. Le principal est ici le législateur, et l'agent est le service public. Dans le cas des médias, cette fonction d'agent est la plus idéale qui soit car le principal accorde pleine liberté à l'agent pour déterminer lui-même ce qu'il compte faire tout en lui garantissant le financement l'augmentation des coûts induits grâce à l'augmentation de la redevance.

La régulation a ainsi contribué fortement à cimenter l'ancien monopole des chaînes publiques et par là même à limiter la concurrence. Cela ne vaut pas seulement pour la conception et la réalisation des programmes, mais également pour leur distribution. L'extension de la couverture technique des chaînes privées a longtemps été inhibée pour protéger le service public. Cela se traduit par un 'monopole' d'attribution des canaux sur les réseaux câblés, exercé par les Landesmedienanstalten, comme l'a rappelé la Commission des monopoles. Or quand un investisseur se trouve face à un tel monopole, il est naturellement peu enclin à investir dans les réseaux câblés, ce qui inhibe leur extension comme leur numérisation. 
I. Bourgeois : Vous voulez dire que la règle du must carry crée des entraves à la concurrence?

J. Heinrich : Oui, puisque cette règle ne signifie rien d'autre qu'une limitation de facto des voies de distribution pour les concurrents des chaînes publiques et s'assimile donc à une limitation de la concurrence dans le câble. Une telle règle n'est justifiée que lorsqu'elle permet de corriger les dysfonctionnements du marché, et seulement dans le cas de biens publics. Et même avec la meilleure volonté du monde, je vois mal comment faire entrer dans cette catégorie un grand show télévisé de type Wetten, dass..., même s'il est produit par le service public (ZDF).

\section{France : un lent et difficile glissement de la loi vers la régulation}

A. Lancelot : Je reviendrais volontiers sur les problèmes de réglementation et de régulation en France, qu'il s'agisse de la concurrence ou du pluralisme des médias. Ce sont des problèmes différents, même s'ils sont liés à un moment ou à un autre. Dans les deux cas, la France est passée lentement d'un domaine de pure réglementation à un domaine où coexistent la réglementation et la régulation.

La pure réglementation dans le secteur de la presse résultait du fait que la loi seule fixait les règles pour le respect du pluralisme. Quant au domaine de la radio et de la télévision, il relevait du monopole public, et donc directement de la réglementation du pouvoir gouvernemental, car ce n'était pas le parlement, mais l'autorité ministérielle qui avait les pleins pouvoirs sur les médias audiovisuels.

Comme l'a très bien expliqué Francis Balle tout à l'heure, à partir de 1982, sous la pression très forte de François Mitterrand, il a été décidé de casser le monopole public de l'audiovisuel, qui n'était que légèrement atteint par les postes périphériques en ce qui concerne la radio, mais qui était néanmoins très fortement installé en citadelle publique. La création d'une " haute autorité », relevant de ce qu'on appelle une autorité administrative indépendante, a été un grand moment de respiration. Pour la première fois, on a coupé le fil direct entre le gouvernement et les médias, et introduit une régulation, c'està-dire le fait que les règles ne soient pas dictées par le pouvoir politique ou par la loi, mais soient dans une certaine mesure ajustées par des 'spécialistes' et des 'arbitres' qui pouvaient prendre position en entrant dans l'esprit même des acteurs. Cela a beaucoup changé les choses. Progressivement, cette régulation s'est établie dans de plus en plus de secteurs, si bien qu'on se demande aujourd'hui s'il n'y a pas autant d'autorités administratives indépendantes qu'il y a d'administrations. Ce qui peut être fâcheux, car à partir du moment où cette formule tend à se multiplier, elle risque de se diluer et de perdre sa capacité d'être un vrai régulateur, combinant à la fois l'empathie vis à vis du secteur considéré et la distance du 'bon père de famille'.

Mais quoi qu'il en soit de cette petite dérive, il faut voir dans la montée en puissance de la régulation une évolution très importante de la République française qui, dans une large mesure, tend à se démocratiser en faisant autant et plus attention aux acteurs du terrain qu'à l'acteur suprême. Ces progrès lents, mais importants, de la démocratie sont à rapprocher de la montée en puissance du Conseil Constitutionnel qui a imposé pour la première fois depuis 1789 l'idée qu'il pouvait y avoir une instance au-dessus de la loi dans le pays qui a remplacé l'absolutisme du roi par l'absolutisme de la loi. Car il ne faut pas oublier que nous n'avons pas supprimé l'absolutisme en France, nous l'avons 
seulement retourné comme un gant. Cet absolutisme de la loi, donc du Parlement qui la vote, est ce dont la République a le plus souffert depuis plus d'un siècle et demi .Il est très sain que, peu à peu, cet absolutisme commence à disparaître au profit d'institutions de régulation.

La montée de la régulation s'affiche également dans le domaine des concentrations, et pas seulement dans le domaine plus politique du pluralisme : le Conseil de la Concurrence est une autorité autonome, et non un secteur du Ministère des Finances. Le malheur est qu'on ne soit pas allé plus loin, qu'on n'ait pas donné à cette autonomie les dimensions qu'elle aurait dû prendre. Aujourd'hui, nous devrions renforcer les compétences du Conseil de la concurrence, car il peut encore arriver qu'il ne soit pas saisi par le ministre. Il faudrait que la saisine soit automatique ou que le Conseil puisse s' autosaisir le cas échéant, pour qu'il ne risque pas de devenir une autorité simplement consultative.

J. Heinrich : Le Conseil de la concurrence est à peu près l'équivalent de notre Commission des monopoles. Pourquoi n'y a-t-il pas en France d'Office des cartels qui soit indépendant et qui observe où se situent les restrictions de concurrence en pleine autonomie, tel un policier?

A. Lancelot : La France est encore une terre de commandement qui estime que c'est le ministre, et lui seul, qui prend la décision, sans expliquer pourquoi il la prend. C'est finalement comme cela que ça se fait, et souvent dans le silence : on ne sait pas comment ni pourquoi. Voilà pourquoi nous pensons qu'il serait essentiel qu'il y ait un renforcement des pouvoirs du Conseil de la concurrence, et aussi du CSA, qui n'a pas eu tous les pouvoirs qu'il devrait avoir, par mauvaise volonté du Parlement et plus encore du Gouvernement, qui n'a jamais pris les décrets d'application nécessaires pour que le CSA puisse jouer son véritable rôle. On l'en a empêché.

\section{"L'infrastructure technologique l'emportera sur la superstructure idéologique »}

Il serait évidemment très utile qu'il y ait des relations plus étroites entre le Conseil de la concurrence et le CSA parce que c'est à ce niveau-là que résident les problèmes. Par exemple, le CSA a-t-il le droit et les moyens de revenir sur une autorisation qui a été donnée au niveau du Conseil de la concurrence ? Peut-on séparer complètement un problème de concentration et un problème de pluralisme ? Il serait sans doute souhaitable que les deux autorités puissent travailler ensemble, voire faire partie d'un même ensemble. Mais nous n'en sommes pas encore là, enfermés que nous sommes encore dans un énorme système bureaucratique.

Car notre secteur public est encore loin d'être régulé. Il est dommage, par exemple, que le CSA ne puisse intervenir dans le secteur public que pour nommer le président des chaînes et qu'il n'y ait pas un vrai cahier des charges pour les chaînes publiques comme il y en a pour les chaînes privées. Je ne vois pas pourquoi on ne devrait pas demander aux chaînes publiques d'avoir les mêmes obligations que les chaînes privées. On estime sans doute que, puisqu'elles sont publiques, elles sont parfaites de naissance, exemptées de tout pêché originel. Malheureusement, ce n'est pas pour ça qu'elles sont satisfaisantes. Comme on le voit, le réformateur ne manquera pas de pain sur la planche.

Mais il sera peut-être devancé par les conséquences de la révolution technologique. Car ce qui paraît le plus porteur aujourd'hui, c'est le côté destructeur de l'évolution 
technologique. On voit apparaître dans le paysage des acteurs, qu'on n'imaginait pas du tout comme des gens importants, s'imposer dans le vieux musée de la presse écrite ou la grande cathédrale de l'audiovisuel public qui paraissaient intouchables. On voit apparaître de nouveaux acteurs conscients de leurs atouts techniques et économiques comme les fournisseurs d'accès à Internet, des gens qui, à 25 ans, sont déjà millionnaires parce qu'ils ont inventé et mis en place un petit système d'accès à Internet, des entrepreneurs qui réussissent, qui inventent, qui trouvent des solutions qu'on n'imaginait pas, et qui vendent le triple play, alors que personne n'imaginait qu'on puisse avoir à la fois la télévision, le téléphone et Internet pour une somme modique (deux fois et demi de moins que Le Monde par mois).

Ces entrepreneurs de la nouvelle génération montrent qu'on peut changer complètement le paysage médiatique. Mais, derrière ceux qui ont ouvert la voie se profilent déjà de plus gros poissons : les sociétés de télécommunications notamment, qui sont encore plus riches que les fournisseurs d'accès. Il faut bien se rendre compte que si l'on compare la capitalisation relativement modeste de Bouygues (financier de TF1) - représentée dans l'imaginaire français comme étant un énorme capital - par rapport à celle de Vodafone, on comprend tout de suite que nous ne sommes pas dans le même monde. Pour ne rien dire de Google, où on entre dans un monde encore plus étrange.

Devant cette révolution, de nouvelles procédures de régulation seront nécessaires. On ne pourra le faire que si on a l'empathie nécessaire pour comprendre ce qui se passe, entrer dans les raisons de tout ce nouveau monde pour tenter de réguler avec lui une révolution qu'il ne s'agit pas d'empêcher. Je dis cela parce qu'aujourd'hui la plupart des acteurs des médias ne rêvent que d'une chose, c'est que la révolution technologique s'arrête. S'ils pouvaient l'interdire par décret, ils le feraient immédiatement. Bien que je ne sois pas marxiste, je suis totalement convaincu que l'infrastructure technologique l'emportera sur la superstructure idéologique !

\section{Concilier approches économique et constitutionnelle - mais comment ?}

D. Schmidtchen : La technologie continue d'évoluer, et pas seulement en France. Le numérique, la diffusion d'Internet et l'intégration verticale des opérateurs de programmes qui absorberont les câblo-opérateurs remettent en question les structures établies et amènent une intensification de la concurrence. Les opérateurs historiques s'opposent naturellement de toutes leurs forces à ces mutations. Mais tout comme est tombé le monopole dans les télécommunications, qu'il va tomber dans l'électricité ou dans le segment des pharmacies, l'audiovisuel sera libéralisé à plus ou moins brève échéance. La pression viendra de Bruxelles ou, si les Etats membres n'adaptent pas leur régulation, comme dans le cas de l'Allemagne, de la Cour européenne de justice à Luxembourg. Rien ne pourra arrêter le mouvement de la dérégulation.

J. Heinrich : Les économistes parlent de progrès technologique ; les sociologues disent : mutations technologiques. Nous sommes convaincus qu'il s'agit d'un progrès car ces mutations réduisent les coûts et offrent de nouveaux produits qui sont mieux à mêmes de satisfaire les préférences des consommateurs.

Mais il faut être prudent lorsqu'on établit une analogie entre la concurrence sur le marché des biens et la concurrence sur celui de l'opinion. Si Berlusconi était un marchand de saucisses, il n'y aurait rien à objecter à cette fusion d'un point de vue économique. 
Mais quand il s'agit d'opinions, de contenus, d'information, la circonspection est de mise pour un économiste. Non seulement ces questions n'entrent pas dans nos compétences, elles concernent aussi et avant tout la protection de la culture nationale.

I. Bourgeois : Peu importe dès lors, semble-t-il, que la régulation visant à garantir le pluralisme s'applique au niveau du capital des opérateurs - signifiant alors pluralité des acteurs - ou au niveau des parts d'audience - dans une perspective de pluralité ou de pluralisme des opinions. Malgré les immenses différences systémiques entre la France et l'Allemagne sur l'approche de la concurrence, au niveau du marché des services comme de l'éventail des opinions, il apparaît à l'issue de ce tour de table que les défis qui se posent à la régulation, parce qu'ils sont de même nature, appellent aujourd'hui avant toute chose une réflexion de fond sur la manière d'aborder la fonction des médias alors que les structures établies depuis bientôt un demi-siècle appartiennent à l'histoire. Or les mêmes différences systémiques rendent visiblement plus nécessaire que jamais la confrontation des fondements sur lesquels sont ou étaient établies les règles visant à interdire les monopoles, qu'ils soient économiques ou qu'ils concernent l'espace public.

Poursuivons donc avec la salle le débat sur la question de fond qui rend si difficile la conciliation du droit économique, des sciences de la communication et du droit constitutionnel.

Victor Henle (dans la salle) : La prise en considération de l'opinion sous l'angle purement économique me gêne beaucoup. L'opinion n'est pas simplement liée à l'information. L'opinion est aussi synonyme de culture, de socialisation. Un exemple : il y a une dizaine d'années, le gouvernement indien avait étudié l'effet de MTV sur la culture villageoise dans un certain nombre de régions. Il a été constaté que, en l'espace de très peu de temps, cette culture avait complètement disparu chez les jeunes spectateurs de la chaîne musicale. On ne peut pas considérer cet exemple-là uniquement dans la perspective du "marché de l'opinion ». Et pourtant, l'information est bel et bien un marché aussi, soumis aux lois de la concurrence...

\section{Pluralisme et/ou diversité culturelle?}

M. Lancelot, j'ai lu une partie intéressante dans votre rapport intitulée, si mes souvenirs sont bons : «le pluralisme et la diversité culturelle ». Comment voyez-vous actuellement le rapport entre pluralisme et diversité culturelle ? Et comment garantir la diversité culturelle en limitant la concentration dans les médias ? D'autres mesures s'imposentelles par-delà l'éventail classique de la régulation ?

A. Lancelot : Il n'est pas simple de garantir la diversité culturelle. Mais, au fond, nous voyons bien que cela relève un peu du même processus que pour la garantie de la diversité des opinions politiques.

Pour ce qui est de la diversité culturelle dans le cas français, nous sommes en face d'un Etat qui a très longtemps considéré la reconnaissance de communautés sousétatiques comme une attaque à l'unité, voire à l'universalité, de la Nation, mais qui a de plus en plus de mal à brider les aspirations décentralisatrices. Ici encore, s'est mis en place un processus lent mais régulier de démocratisation par lequel la République tend à reconnaître progressivement la spécificité d'un certain nombre de communautés : les régions de la métropole ou de l'Outre mer, les populations immigrées et les religions qu'elles pratiquent, les minorités sexuelles, voire les handicapés, etc. L'évolution, 
d'abord assez lente, a tendance à s'accélérer, avec peut-être autant de résignation que de véritable ralliement dans une bonne partie de la population. Mais la diversité culturelle s'inscrit progressivement dans les faits et dans les têtes.

Prenons l'exemple des langues régionales que la plupart des Français regardent avec mépris : il existe aujourd'hui une chaîne de télévision, TV Breizh (en français Télé Bretagne), dans laquelle, lorsqu' on vous demande de choisir votre langue, vous n'avez pas le choix entre l'allemand, l'italien ou l'espagnol, mais entre le français et le breton. Il faut dire que ce n'est pas triste de regarder les enquêtes du lieutenant Colombo ou les westerns en breton. C'est une avancée qu'on n'aurait pas imaginée il y a seulement 20 ans et qui illustre la reconnaissance progressive de la diversité culturelle. Evidemment, cette reconnaissance ne remplace pas la défense de la culture française, qui est un élément clé de la diversité culturelle telle que la voit le gouvernement français, c'est-à-dire l'affirmation que la France doit être toujours capable d'offrir aux Français des oeuvres qui ne soient pas simplement importées des États-Unis d'Amérique.

La protection de la diversité culturelle, c'est encore et d'abord la protection du Français vis-à-vis des peuples qui l'entourent. Je ne pense pas ainsi, mais je reconnais que ce nationalisme culturel a tendance à diminuer dans les jeunes générations. En dehors de la droite la plus conservatrice, la diversité culturelle est de plus en plus présente au sein de la communauté nationale.

V. Henle : Il s'agit pour moi d'une question centrale. J'y vois même trois aspects : la protection contre une opinion en position dominante, la préservation de la culture et, enfin, une contribution à la socialisation, c'est-à-dire la transmission de valeurs qui constituent le lien social, et en tête desquelles je place la liberté. Je ne suis pas certain que le service public de l'audiovisuel soit encore en mesure de transmettre ce capital social, comme disent les économistes, mais il doit s'y évertuer. Cela étant, j'insiste sur le fait que la diversité n'est pas un problème d'offre mais de demande. L'offre des chaînes publiques est certes excellente, mais personne ne la regarde. La concurrence ne suffit pas à atteindre les trois objectifs de pluralisme ou diversité que j'ai évoqués.

\section{Garantir la production de biens méritoires pour préserver le lien social}

Uwe Kamman (dans la salle) : La théorie de la convergence au niveau des programmes, autrement dit : du nivellement vers le bas, n'est qu'une une légende créée par leurs concurrentes pour porter préjudice aux chaînes publiques. Mais, plus important, elles produisent des biens méritoires. Et leur apport en ce qui concerne la socialisation ou la création de l'identité d'une société démocratique a été particulièrement important dans la République fédérale d'après-guerre. Bien sûr, elles ont connu une situation de monopole qui limitait la possibilité pour le spectateur de recourir à d'autres sources. Même un show comme Wetten, dass... contribue à entretenir le lien social.

Et souvent, les émissions considérées comme 'triviales' ont un impact de socialisation plus fort que l'information, bien qu'il ne soit pas réellement possible de le mesurer avec précision. Il reste une spécifité du service public, et celui-ci a un rôle central à jouer dans la société. Certes, comme Jürgen Heinrich, je constate que la demande a évolué. Et que l'audiovisuel n'est pas seulement un média d'offre, c'est aussi un média de demande, bien qu'il y ait de nombreux consommateurs qui ne voient pas la richesse de cette offre et n'en profitent donc pas. 
J. Heinrich : Il est indéniable que les biens méritoires ne peuvent être produits de manière efficiente par le marché. Et il n'y a rien à redire au fait que ceux-ci soient proposés par les chaînes publiques allemandes. Je constate simplement une tendance, à savoir que ces biens sont offerts aujourd'hui de plus en plus par les chaînes thématiques et de moins en moins par les généralistes. Or le service public avait été créé justement pour les produire...

Deuxième point : comme vous, j'estime Wetten, dass... a une fonction socialisatrice, car l'émission apprend, sous la forme d'un jeu, que la vie en communauté est régie par des règles. On le voit très bien quand on regarde l'émission avec des enfants. Il n'en reste pas moins la question de savoir si nous avons réellement besoin d'un service public pour produire ce genre d'émissions. Aujourd'hui, les chaînes publiques n'ont plus qu'une fonction de complément. Il en allait autrement lors de leur création, et le Tribunal constitutionnel fédéral avait alors défini leur fonction dans une approche maximaliste. Il n'est pas certain que, face aux évolutions constatées, il maintienne cette position.

Dernier point, au sujet de la demande. Je suis très influencé par les philosophes écossais et les pères de l'économie de marché allemande qui croient en une organisation spontanée du marché, contrairement au Français qui croient en Descartes, en la planification. Normalement, les préférences des individus vont vers cette auto-organisation du marché, puisqu'elle permet d'assurer une offre satisfaisante et diversifiée. Or certains cherchent à établir un autre type d'offre et doivent dans ce cas légitimer cette prescription. Dans certains domaines, cela peut se concevoir: la scolarité obligatoire, par exemple, est indispensable à la socialisation ; dans ce cas, la légitimation d'une réglementation est directe.

Mais quand vous dites que la réalisation d'un show comme Wetten, dass... entre dans la mission du service public, alors vous procédez à une légitimation par substitution : pour faire accepter à l'opinion un bien utile pour le lien social, il faut l'habiller, le 'vendre', de manière à ce que le citoyen ait envie de le consommer. Marketing et publicité maîtrisent à merveille cette manière de procéder. Mais encore faut-il que l'équilibre entre le bien et son argument de vente ne soit pas brouillé ; il ne faut par exemple pas prendre la jolie dame sur la publicité pour la voiture à laquelle elle prête son image. Cette confusion est plus courante qu' on croit dans le marketing, et nous devrions nous demander si nous ne sommes pas en train d'en commettre une semblable à propos de la télévision publique.

Ultime remarque : à mes yeux, MTV aussi relève de la culture. La chaîne a sa place dans une organisation des médias visant la garantie du pluralisme; et les régulateurs ne s'y sont pas trompés.

Plutôt que la concentration, le vrai problème ne serait-il pas notre malthusianisme ?

Jean-Louis Missika (dans la salle) : Je voudrais prolonger l'analyse d'Alain Lancelot sur l'apparition des nouveaux acteurs grâce aux nouvelles technologies dans le champ de l'audiovisuel. Je parle pour la France, mais je me demande si ce n'est pas la même chose en Allemagne : le problème majeur n'est pas celui de la concentration des médias, c'est celui du malthusianisme. Le malthusianisme nous amène à une question essentielle, qui est que, en général, il est commandé par la 'sur-réglementation'. 
La position dominante d'une chaîne comme TF1 sur le marché français s'explique à mon avis, contrairement à ce que disent ses dirigeants, beaucoup moins par leur talent de managers que par l'existence d'une réglementation qui protège le fort contre le faible. A chaque fois qu'il y a une innovation soit technologique, soit de contenu, les politiques en France disent qu'il faut accroître la réglementation, mais ils ne disent pas quelle est la réglementation qu'il faut supprimer pour la remplacer par une nouvelle, ils proposent simplement de rajouter une couche réglementaire. Et aujourd'hui, ce n'est pas ma collègue du CSA qui me contredira, circuler dans la jungle réglementaire française de l'audiovisuel est un parcours du combattant. Je défie n'importe quelle petite chaîne de télévision émettant sur le câble ou le satellite de savoir exactement quelles sont ses obligations et ce qu'elle doit faire pour être conforme à la réglementation.

Internet, l'apparition des nouveaux médias et de la télévision sur ADSL, ont représenté une bouffée d'oxygène extraordinaire dans ce pays. La France est un leader mondial en matière de télévision sur ADSL. Parce que la régulation a été très bien faite par l'ARCEP, la France est un pays dans lequel l'abonnement au triple play, c'est-à-dire télévision, téléphone illimité et Internet haut débit, est à 29,90€. De ce fait, la France est un pays dans lequel il y a près de $50 \%$ des foyers qui sont équipés en haut débit; et il y a de ce fait également des initiatives qui sont prises en matière de création audiovisuelle sur le haut débit et qui sont exceptionnelles parce qu'il y a un cercle vertueux qui est en train de se constituer.

Je suis tout à fait d'accord avec Alain Lancelot: les médias audiovisuels français rêvent d'assassiner à la fois les Internet service providers et même le numérique. L'analogique (et la pénurie qu'il crée) est une bénédiction pour eux ; la bataille qu'ont menée les grands médias contre les initiatives du CSA en matière d'appels à candidatures pour les télévisions numériques terrestres en est une excellente illustration. Ils ont quand même réussi une bataille de retardement qui a duré plusieurs années. De ce point de vue, le CSA a été très courageux de mener à son terme l'attribution des fréquences TNT. Ma question est : ne faudrait-il pas concentrer nos réflexions sur le malthusianisme plutôt que sur la concentration des médias ?

\section{« Ne demandons pas aux médias de faire ce qu'ils ne peuvent pas faire »}

Francis Balle (dans la salle) : Lorsque nous nous sommes réunis avec Alain Lancelot dans le cadre de la commission qu'il présidait, nous sommes parvenus à la conclusion que la France ne souffre pas d'un excès de concentration mais d'une insuffisance de concentration, et c'est précisément parce que nous n'avons pas suffisamment de groupes forts et puissants que la diversité est trop limitée et donc que le pluralisme est atteint.

Ma seconde remarque concerne le "marché de l'opinion », une expression effectivement un peu difficile. La diversité ne se décrète pas, et on ne peut pas demander de boire à un âne qui n'a pas soif. Ce n'est pas par les médias qu'on arrivera à plus de diversité, c'est par l'éducation, tout simplement.

$\mathrm{Ne}$ demandons pas aux médias de faire ce qu'ils ne peuvent pas faire. Quand on déplore que les gens ne savent pas lire, on dit que c'est à cause des médias, quand on déplore l'absence de culture de nos contemporains, on dit encore que c'est la faute des médias. Pas du tout, c'est la faute d'autres institutions dont c'est la vocation première. 
Dites-moi comment fonctionnent vos écoles, vos autres institutions de socialisation et je vous dirai ce que vous avez comme médias.

Ne demandez pas aux médias de faire de la diversité. Demandez à la société de pouvoir s'épanouir librement et d'avoir sa propre diversité. Vous aurez alors des médias qui seront divers. Ne trichons pas avec la réglementation. Et comme l'a judicieusement fat remarquer Jean-Louis Missika: on a voulu tellement surajouter des réglementations qu'on a abouti exactement au résultat inverse de celui qu'on cherchait.

I. Bourgeois : De ce point de vue, la France et l'Allemagne se retrouvent donc confrontées au même problème, malgré toutes leurs différences d'approche du pilotage du marché ou des normes et règles à adopter pour garantir la diversité et le pluralisme des opinions. 


\section{Debat $\mathbf{N}^{\circ} 4$}

SEgmentation DE L'OFFRE, INDIVIDUALISATION DE LA RECEPTION, ATOMISATION DE LA SOCIETE.

Quelles bases de légitimité pour une régulation des médias? 



\title{
DEBAT $N^{\circ} 4$
}

\section{SEGMENTATION DE L'OFFRE, INDIVIDUALISATION DE LA RECEPTION, ATOMISATION DE LA SOCIETE. Quelles bases de légitimité pour une régulation des médias?}

\author{
EXPOSE INTRODUCTIF : Jean-Louis Missika (IEP) \\ INTERVENANTS : \\ Jean-Louis Missika (IEP) \\ Jacques Rigaud \\ Norbert Schneider (LfM)
}

Débat animé par Andreas Stopp (Deutschlandfunk)

Pour nos modèles de société, la numérisation représente une révolution d'une ampleur comparable à celle de l'invention de l'imprimerie. Elle mène notamment à une "fracture numérique " entre, d'un côté, une jeune génération familière de l'univers du Web 2.0, et de l'autre, une classe politique pour qui l'univers numérique reste mystérieux.

Ces évolutions, qui s'accompagnent de changements importants dans l'organisation du marché médiatique, soulèvent de nombreuses questions : comment contrôler des acteurs multipositionnés à la fois géographiquement et sectoriellement? Comment contrôler, au besoin, une offre pléthorique, extrêmement diversifiée et d'origine souvent individuelle ? Comment protéger la jeunesse, la vie privée ou la propriété intellectuelle ? Comment protéger le citoyen des rumeurs et de la désinformation?

La révolution technologique déplace le pluralisme au niveau du consommateur, ce qui présente de nouveaux risques pour la société et la démocratie. Pour les endiguer, l'existence de grands opérateurs, capables d'assumer une mission fédératrice dans une société menacée d'atomisation, est plus indispensable que jamais. Mais cela ne suffit pas. Pour être capable de s'orienter dans la jungle de l'offre, le consommateur et citoyen doit disposer de compétences médiatiques - compétences-clefs dans l'ère du numérique et la société de l'information.

Les mutations en cours sont imprévisibles. Mais une nouvelle cohérence va finir par s'esquisser. De nouveaux systèmes de repères devront se développer pour permettre l'orientation dans la masse d'information disponible. Nos modèles classiques de régulation sont impuissants face à ces évolutions. C'est au système éducatif qu'il reviendra d'assumer en quelque sorte le rôle de régulateur : en délivrant cette capacité d'orientation dont aura besoin le citoyen et consommateur, en expliquant et commentant l'offre pour lui redonner du sens et recréer la confiance. 



\title{
EXPOSE INTRODUCTIF
}

\section{Heurs et malheurs de la révolution numérique}

\author{
Jean-Louis Missika
}

Nous vivons une révolution d'une ampleur considérable. Pour moi, c'est une révolution qui a la même ampleur que la révolution de l'imprimerie ou la révolution industrielle. Comme toute révolution, elle a plusieurs dimensions : une dimension technologique, économique, socioculturelle, politique, et comme toute révolution, elle crée des angoisses et des espoirs. Il faut savoir que la peur du nouveau média est une constante de la vie politique des pays démocratiques et même des pays non-démocratiques. La peur du nouveau média déstabilisateur, incontrôlable, a conduit des pays comme la France, qui est pourtant une démocratie, à instaurer un monopole d'Etat sur la radio et sur la télévision pendant 40 ans.

Avec Internet, l'angoisse du législateur et du politique est aggravée par le fait que c'est un média insaisissable, protéiforme, global, qui se moque des logiques de territoires et donc des Etats-Nations. Le mot qui caractérise la période que nous vivons est l'incertitude.

Incertitude technologique : nous passons notre temps, surtout les gens d'un certain âge comme moi, à naviguer face à des acronymes qui sont difficiles à comprendre : IP, ADSL, UMTS. A peine a-t-on compris ce que c'était que le WiFi qu'on nous explique qu'il y a le WiMax, naturellement différent du WiFi. Donc comme toute révolution technologique, c'est une succession de ruptures technologiques. Et chacune d'entre elles entraîne un bouleversement du marché.

Incertitude socioculturelle, parce qu'une technologie, ce sont des outils que les gens doivent s'approprier et qui ont ceci de particulier qu' on doit inventer les usages. Ces usages s'inventent progressivement, et parfois il y a de grosses surprises. Par exemple : qui aurait pensé que le 'social networking' serait un must de la révolution Internet?

Incertitude politique, parce que si on est d'accord pour légiférer, réguler, réglementer, on ne sait ni quoi ni comment. Et on verra que les réponses à ces deux questions ne sont pas évidentes.

\section{Un univers d'incertitudes technologiques...}

Commençons par les incertitudes technologiques. On a vu dans les débats précédents que le principe de base de la révolution que nous vivons est le numérique. Mais il y a aussi le haut débit, le wireless, le protocole Internet, c'est-à-dire une certaine manière de 'packager' les données pour les transporter sur les réseaux, et il y a la profusion des terminaux (consoles de jeux, PC, téléphone, PDA, téléviseur). Encore pour quelque temps, le téléviseur sera le moyen de recevoir la télévision, mais on ne sait pas pendant combien de temps. Microsoft vient d'annoncer une offre de téléchargement de films et 
de séries sur la console de jeux XBOX. Il y a aussi technologiquement les logiciels de partage, les moteurs de recherche.

Nous sommes dans un moment schumpetérien de destruction créatrice. Pour les gens qui s'inquiètent de voir ces milliers d'entreprises qui sont crées et qui disparaissent du jour au lendemain, je rappellerai simplement qu' au moment de l'invention de l'automobile, il y avait plus de 10000 fabricants d'automobiles rien qu'aux Etats-Unis, qu'en une cinquantaine d'années, le nombre s'est réduit à trois et qu'aujourd'hui, il y en a deux sur les trois qui sont en train de mourir.

La caractéristique principale de cette révolution est l'effondrement des coûts de diffusion. Aujourd'hui, une chaîne de télévision comme TF1 paie environ 100 millions $€$ pour sa diffusion sur le hertzien analogique ; or pour diffuser une offre audiovisuelle sur le Web, c'est quelques milliers d'euros seulement. Si vous créez une chaîne de télévision en France et que vous demandez à Free de la diffuser, celui-ci le fait gratuitement, Free étant un fournisseur d'accès à internet.

... qui génère de grandes incertitudes socioculturelles

J'en viens aux incertitudes socioculturelles : quand une nouvelle technologie apparaît, en général, il y a une projection de l'ancien média sur le nouveau média. Quand la radio est née, on a fait le journal parlé - d'ailleurs les journalistes ne voulaient pas annoncer les nouvelles à la radio, et donc ce sont des speakers, qui n'étaient pas des journalistes, qui le faisaient : les journalistes écrivaient les papiers et les speakers les lisaient. Quand la télévision est apparue, on a fait de la radio filmée. Quand Internet est apparu, les radios, journaux et télévisions ont pris leur produit et l'ont plaqué sur le nouveau média, mais d'un certain point de vue, c'est déjà l'ère paléolithique. C'est terminé car très vite, Internet s'est émancipé.

Cette émancipation a eu une double dimension. La première était prévisible : comme on peut combiner dans Internet des médias - du texte, de l'image et du son-, les services sont devenus très vite des services multimédias. Quand vous allez sur le site du New York Times ou du Monde, vous vous rendez compte qu'ils n'ont plus grand chose à voir avec le journal papier car vous avez de la vidéo, de l'audio, des chats, blogs, forums. Toutes les technologies potentielles sont utilisées sur ces services. Le journal papier ne peut pas lutter contre cela, il est dépassé par rapport à ce caractère multiple et varié de l'offre Internet.

On est face à quelque chose qui était prévisible mais qui n'en a pas moins un effet déstabilisateur : l'éclatement du business modèle des médias. Les anciens médias (radio, presse écrite et télévision) vivaient sur une combinaison de trois sources de financement: les petites annonces, la publicité et le paiement par le lecteur ou l'abonné. Aujourd'hui, les petites annonces sont littéralement captées par des structures Internet spécialisées dans les petites annonces comme Monster. Il y a donc un 'siphonage', une disparition de la ressource financière des petites annonces pour la presse écrite. La publicité est captée par les moteurs de recherche et par les agrégateurs de contenu. La culture de la gratuité a été développée par Internet. Faire payer une information devient de plus en plus compliqué.

Mais je pense que la plus grande transformation à laquelle nous assistons est le Web 2.0. Les nouvelles générations se sont emparées de cette technologie, de ces nouveaux 
outils pour inventer de nouveaux usages et c'est cela qui a provoqué la fracture numérique puisqu'il y a deux mondes maintenant : il y a les natifs du numérique et nous, les natifs de l'analogique et donc les émigrés du numérique parce que nous ne pratiquons pas les nouveaux usages inventés par cette nouvelle génération.

\section{Le Web.2 fait apparaître la fracture entre médias conversationnels et institutionnels}

Je vais vous donner un exemple d'un effet politique de ce phénomène en France : au moment de la crise du CPE, le premier ministre est venu expliquer le CPE sur TF1, mais les jeunes ne regardent plus TF1. Par contre les gens ont créé une trentaine de milliers de blogs sur Internet qui parlaient du CPE, les services de Matignon ne lisant naturellement pas les blogs. C'est un exemple de ce que peut créer comme malentendus l'apparition de ces nouvelles technologies.

J'ai parlé d'acronymes tout à l'heure : de quoi parle-t-on quand on parle de Web 2.0 ? De peer-to-peer, de blogs, de social networking. Cela concerne aussi bien les services que l'information et le divertissement. On parle de sociétés qui n'existaient pas il y a quelques années : My Space et YouTube et dont la valorisation boursière se chiffre en plusieurs centaines de millions de dollars. Quelques chiffres : une société comme My Space a 80 millions de visiteurs uniques par mois et 276 milliards de pages vues par mois. Une société comme YouTube, qui n'existait pas il y a trois ans : 72 millions de visiteurs uniques par mois 16600000000 de pages vues par mois. Si je prends un acteur uniquement français, SkyBlog : 11559000 visiteurs uniques par mois, 2603000 visiteurs par jour qui viennent tous les jours soit pour poster un texte, soit pour lire les textes des autres, et il y a plus de 3253000000 de pages vues par mois.

Ces chiffres donnent une idée de l'ampleur de la révolution que nous connaissons. Aux Etats-Unis, les 15-24 ans s'informent davantage sur Internet que sur tous les autres médias réunis, et c'est vrai de plus en plus pour les 25-34 ans. Aux Etats-Unis, un citoyen sur deux s'est informé sur Internet lors de la campagne présidentielle de 2004.

Cela montre que le Web 2.0 représente une opposition majeure entre deux catégories de médias : les médias institutionnels d'un côté, et d'un autre côté les médias conversationnels. Hier, au cours de nos débats, nous n'avons parlé que des médias institutionnels. Un média institutionnel est un média créé par une institution politique, une chaîne publique de télévision par exemple. Ce média est lui-même une institution, il a tendance à parler des institutions et à structurer sa relation au téléspectateur ou à l'auditeur de façon inégalitaire : il y a celui qui parle et celui qui écoute. Ces places sont réservées à des gens qui ont un monopole de la parole légitime ; en face d'eux, des gens qui ont une obligation d'écoute.

Le média conversationnel est tout le contraire : l'interaction, le passage indifférencié de la parole à l'écoute et de l'écoute à la parole. Nous sommes en train de passer d'un monde où le nombre, la position et le statut des émetteurs par rapport aux récepteurs était clairement limité, identifié, à un monde où la frontière entre émetteur et récepteur s'estompe, où le nombre d'émetteurs devient infini, où le monopole de la parole légitime disparait. Tout cela a des effets spectaculaires.

Quelques exemples politiques de ces effets spectaculaires : en Grande Bretagne, lors des attentats de juillet, le gouvernement anglais décide de ne pas autoriser les médias à filmer. Les gens dans le métro ont sorti leur téléphone mobile et ont filmé. Quand ils 
sont sortis, ils ont envoyé cela à des amis, qui avaient des blogs et qui ont mis cela sur leur blog. Les images ont fait le tour du monde et tout ce qui a été mis à l'antenne des télévisions et à la une des journaux étaient des images de cette nature, prises par des amateurs, alors même que le gouvernement anglais avait décidé que les professionnels ne devaient pas avoir accès à l'évènement.

Un deuxième exemple, qui a fait couler beaucoup d'encre : pour s'amuser, des soldats américains prennent des photos dans la prison d'Abu Ghraib et se la font passer sur le Web entre eux. Vous savez les effets spectaculaires politiques qu'a eu l'apparition des ces images sur les médias traditionnels. Ce geste, qui était autrefois un geste professionnel (production -édition - diffusion) appartient tellement à la vie quotidienne qu'il se fait presque naturellement et qu'il est pratiqué par des millions de gens qui le font sans s'en rendre compte, parce que cela fait partie de leur vie quotidienne.

Un autre exemple, et assez intéressant : en Liban Sud à l'été 2006, des blogueurs ont démontré que des photos de Reuters et d'Associated Press avaient été retouchées et truquées. Les agences de presse mondiales ont réagi d'abord de façon extrêmement arrogante, puis elles ont été obligées de s'excuser puisque le blogueur était un spécialiste de la retouche photographique et a fait une démonstration absolument impitoyable, que j'ai lue directement sur le Web, du fait que les photos avaient été truquées. Le photographe en question était un local, vraisemblablement proche du Hezbollah qui avait été recruté par Reuters pour éviter que des expatriés se fassent kidnapper.

Tout cela illustre la percée des amateurs, et leur arrivée sur le territoire autrefois réservé aux professionnels. Cela illustre aussi la transformation de la construction des réputations. Je ne vais pas m'étendre sur ce point, j'ai eu l'occasion d'écrire là-dessus. La réputation des journalistes se détache de la réputation de leur journal. La construction des réputations se fait de plus en plus sur Internet, de façon informelle. Ce n'est plus une réputation de l'institution mais une réputation bottom-up. Puis il y a l'apparition de stars du blog, de la même façon qu'il y a eu des stars du journalisme ou de la télévision.

Incertitudes politiques, ou de l'impossibilité de réguler un processus mouvant et mondial

J'en viens aux incertitudes politiques et aux problèmes que tout cela pose à la question de la régulation d'Internet et des nouveaux médias. Je vais me contenter de lister les problèmes parce que je n'ai pas de solution, nous sommes dans l'incertitude.

Le premier problème que je vois, c'est que les acteurs principaux du Web, tous ceux que j'ai cités jusqu'à présent, ne sont pas des médias. Ce sont des moteurs de recherche, comme Google, ce sont des portails, comme MSN, Orange ou Yahoo, ce sont des plateformes d'agrégation de contenus ou de social networking comme YouTube, My Space, Sky Blog, ce sont éventuellement des outils de personnalisation de services. Il n’y a pas d'éditeur au sens juridique du terme, et la responsabilité éditoriale est partagée entre celui qui poste sa vidéo et celui qui lui donne les outils de la diffusion et de l'édition. S'il n'y a pas d'éditeur, quel est le rôle de l'instance de régulation ? C'est une vraie question.

Deuxième problème, ces acteurs captent une part importante de la ressource publicitaire alors qu'ils ne produisent pas de contenu. Or, jusqu'à présent, le modèle de base d'un média était que les ressources publicitaires servaient à financer la production de 
contenus. Aujourd'hui, ce sont des entités indépendantes qui d'un côté produisent les contenus et de l'autre côté captent la ressource publicitaire. Hier, Google a proposé aux principaux journaux américains, c'est-à-dire le New York Times, le Washington Post, le Los Angeles Times, etc., de s'occuper de leur régie publicitaire.

Pour vous donner l'ampleur du phénomène, voici un chiffre : la plupart des instituts estiment qu'en 2010, Internet générera 50 milliards $\$$ de ressources publicitaires et que la moitié de cette somme sera captée par les moteurs de recherche. Les prévisions sont faites pour être démenties, je le sais bien, mais cela préfigure ce qui va se passer du point de vue de la chaîne de valeur dans les cinq ans qui viennent.

Troisième problème, qui pourrait paraître anecdotique mais qui est loin de l'être : ces acteurs ne sont pas nationaux. Or un législateur, un régulateur intervient sur un territoire national. Ce sont des méga-médias mondiaux : aujourd'hui Google est présent sur l'ensemble de la planète, MSN et Yahoo aussi. Si un législateur décide de créer une nouvelle forme de régulation d'Internet, comment sera-t-elle appliquée ?

Quatrième problème : la réglementation suppose le contrôle. Comment contrôler des centaines de millions de sites, de blogs, et de social networking ? C'est quasi impossible. Aujourd'hui, un site comme SkyBlog a 5 millions de blogs, plusieurs dizaines de milliers de nouveaux textes qui sont postés chaque jour. Bien évidemment, on peut faire du contrôle automatisé, on peut imaginer des moteurs de contrôle comme il y a des moteurs de recherche. Il n'empêche que, pour un régulateur comme pour un législateur, il est extrêmement difficile de dire : nous créons une réglementation et nous allons contrôler le contenu de ces médias, d'autant plus, comme on l'a dit hier, qu'il y a toujours eu dans les démocraties une différence entre ce qui était le multipoint et le point-à-point, et que les blogs et autres relèvent d'une logique conversationnelle, de point-à-point, c'està-dire de quelque chose dans quoi l'Etat ne devrait pas avoir le droit de s'immiscer.

La régulation passe également par l'octroi de licences, d'autorisations à émettre. Là aussi, si la télévision par Internet se déploie, il n'y aura plus de licences. Il ne sera plus nécessaire de demander une autorisation pour créer une télévision sur Internet.

Ensuite, il y a le problème du rythme du changement. Les ruptures technologiques se succèdent tous les ans. Pour faire voter une loi sur l'audiovisuel en France, il faut compter cinq ans, et encore. Donc la réglementation est toujours en retard. On a un embryon de solution pour ce problème, qui a été évoqué par Alain Lancelot hier, c'est de donner plus de pouvoir juridictionnels aux instances de régulation indépendantes. Enfin, dans un pays comme la France, pour le coup, ce serait une révolution politique. On voit mal comment des institutions aussi respectables que le Conseil d'Etat ou le Parlement accepteraient que le CSA ait un pouvoir juridictionnel en fonction de l'apparition de nouvelles technologies.

\section{Un risque majeur : l'incompréhension du monde politique}

Le septième problème, et c'est peut-être celui qui me préoccupe le plus, est la compréhension des enjeux par les acteurs politiques. L'ignorance de la classe politique à l'égard de tous ces phénomènes est proprement insondable. On a beau essayer de faire de la pédagogie, on n'y arrive pas. Non seulement ce sont des immigrés du nouveau pays, mais ce sont des immigrés qui refusent d'apprendre la langue du nouveau pays, sans doute parce qu'ils se rendent compte que ça atteint leur pouvoir et que ça change 
profondément la donne politique. Donc on assiste à des choses surréalistes : les députés français se sont ridiculisés il n'y a pas si longtemps, en voulant absolument imposer une législation sur l'interopérabilité parce qu'ils croyaient qu'un logiciel interopérable était la même chose qu'un logiciel libre. Quand ils ont découvert - encore que je ne sois pas sûr qu'ils l'aient découvert - que Microsoft avait passé un accord avec toute une série d'acteurs du libre, ils se sont peut-être rendus compte que parler d'interopérabilité, ce n'est pas nécessairement approprié. Et puis plus récemment, quand ils ont voulu imposer la licence forfaitaire pour l'échange de fichiers musicaux sur internet, un de mes amis m'a dit que c'était soit illégal, soit inapplicable et je lui ai répondu : c'est probablement les deux à la fois.

Je pense qu'il y a un immense effort à faire de formation de la classe politique à ces nouveaux enjeux, parce qu'on se rend compte que les enjeux se déplacent. Aujourd'hui, quand on parle de diversité culturelle ou d'exception culturelle ou de pluralisme de l'information, on parle justement du monde fermé des anciens médias.

Mais quels sont les problèmes liés au monde ouvert dans lequel nous entrons ? C'est un problème de désinformation. Il y a des risques réels - on l'a vu à plusieurs reprises que des groupes fassent circuler des informations fausses délibérément (je ne parle pas de la désinformation par inadvertance qui est le lot de la presse traditionnelle). La rumeur construite délibérément est quelque chose d'extrêmement important. Il y a un énorme problème de protection de l'enfance. Il y a un problème de protection de la vie privée : dès que vous allez sur Google, il vous met un cookie dans votre ordinateur et il n'est pas simple, même pour un spécialiste de l'informatique, de se débarrasser de ce type de cookie. Il y a un problème de la protection de la propriété intellectuelle, etc.

Et puis évidemment, le problème le plus sérieux est celui de la légitimité de l'intervention de l'Etat. Est-il légitime de vouloir réguler, réglementer ce qui s'apparente à des conversations, à des initiatives individuelles et qui ne peut pas être considéré de la même façon que de celle dont on doit considérer aujourd'hui un média traditionnel ? J'en arrêterai là car je vois que j'ai listé un très grand nombre de problèmes, et je ne pense pas que la table ronde suffira à les résoudre... 


\section{Discussion}

Andreas Stopp : Je propose d'aborder le débat sur les défis du futur par le biais de l'expérience quotidienne. J'ai sur mon toit deux paraboles. L'une est classiquement orientée sur Astra pour capter les chaînes allemandes. L'autre est pointée dans une direction tout à fait différente. Les voisins se demandent si c'est pour capter Dubai TV ou Al Jazeera. Non, elle est orientée sur le satellite de France Télécoms, ce qui me permet de capter à Cologne M6, TF1, France 2, France 3. Et de temps en temps, je regarde la télévision française. Sur la une, il y a un présentateur qui hurle, sur la deux, il y a un public qui est debout et qui hurle... et je me dis, ça doit être ça, la fin de la télévision. Mais ce n'est pas cela, Jean-Louis Missika, que vous décriviez dans votre ouvrage ainsi intitulé...

J.-L. Missika : ... j’y évoque toutes les transformations que je viens d'exposer brièvement dans ma présentation et je dis que la télévision telle que nous la connaissions est en train de disparaître. Pour moi, ce n'était pas seulement des images qui arrivaient à domicile, mais c'était une programmation, faite par des programmateurs professionnels et une écoute collective avec des rendez-vous. C'était la fabrication du lien social par la télévision.

\section{Le numérique signifie-t-il la fin de la télévision?}

C'est cette fonction qui disparaît sous nos yeux puisque de plus en plus souvent. Il y a ce qu'on appelle dans le jargon la télévision délinéarisée, c'est-à-dire qu'il est possible de voir une émission, mais pas à l'heure où elle est diffusée. Il y a la video on demand, et puis il y a tout ce que j'ai expliqué : la désertion des jeunes à l'égard des médias traditionnels et l'apparition de ces plateformes d'agrégation de contenus et de cette possibilité de produire ou de coproduire des contenus audiovisuels. C'est cela que j'ai appelé «la fin de la télévision ». Mais cela ne signifie pas la fin de l'image, bien au contraire. Le transport et l'arrivée de vidéos à domicile, et même ailleurs qu'à domicile : sur les téléphones mobiles, sur les portables, dans les lieux de travail, cela va se multiplier, il y aura toujours plus de services audiovisuels mais ils s'apparenteront de moins en moins à ce que l'on appelle télévision.

Norbert Schneider : Ces évolutions concernent pareillement l'Allemagne, même si les degrés de pénétration respectifs peuvent varient légèrement dans leur détail.

A. Stopp : Français comme Allemands, nous avons donc à faire face au même problème, ou plutôt au même défi, ou encore à la même opportunité ?

N. Schneider : Il s'agit d'une révolution, certes, mais une autre révolution nous a révélé dans le passé que la noblesse n'en était pas supprimée pour autant... A l'issue de la révolution du numérique, il restera toujours quelque chose de ce monde de l'analogique tel que nous l'avons connu jusqu'à aujourd'hui, mais ce quelque chose aura été transformé, rénové, épuré et anobli. Je ne crois donc pas à la thèse de la mort de la télévision.

Sur ce point, les perceptions allemande et française diffèrent légèrement, si je puis me permettre de généraliser ma pensée à l'approche allemande. Je pense plutôt que la télévision 
va se repositionner sur le marché grâce à un changement de profil. Tout ce dont nous parlons a lieu dans un contexte globalisé, et la globalisation n'induira pas seulement l'individualisation de l'utilisation - c'est elle qui fait la nouveauté d'Internet - mais également la globalisation des contenus. Or curieusement, quand nous apprenons des choses aujourd'hui sur Internet ou sur ses évolutions, nous l'apprenons par la télévision. On peut donc s'attendre à un parallélisme dans les évolutions et une spécialisation des divers médias.

Il sera intéressant de voir comment les contenus mondialisés de ce système par définition global qu'est Internet vont s'adapter à la dimension locale autour de l'utilisateur. Cela est fortement lié à l'utilisation des médias aujourd'hui qui n'est plus - et c'est un fait nouveau - dépendante d'un lieu particulier. Mais comme la communication du Web n'en est qu'à ses balbutiements, il m'est très difficile d'établir des prévisions.

Notre société est aujourd'hui structurée en «milieux », et ceux-ci ne sont plus délimités par des frontières géographiques. Il est donc tout à fait concevable qu'en France et en Allemagne et au-delà se dégagent, au sein de milieux définis par leur type de demande, des similitudes dans la consommation des médias. De même, cette similitude pourrait être un critère de définition de certains types de milieux communs aux Etats-Unis, à l'Inde, à l'Allemagne ou à la France, en un mot, des milieux présents à l'échelle mondiale.

Pour la démocratie, il y a un risque plus grand que les monopoles : la perte d'une éthique...

A. Stopp : Avant de nous projeter dans le futur, revenons au moment-charnière que nous sommes en train de vivre. Quelle est la situation en France ? La télévision y possède-t-elle encore sa force intégratrice ? En Allemagne, les grandes émissions capables de fédérer la nation entière n'existent plus depuis longtemps, à l'exception de ce Wetten, dass... qui a été souvent évoqué dans nos débats. Elles rassemblaient les téléspectateurs devant leur poste à $20 \mathrm{~h} 15$ en Allemagne, à $21 \mathrm{~h}$ en France, ces 'carrefours' des préoccupations de la société. Dans quelle mesure la nation télévisuelle française se rassemble-t-elle encore le soir devant un programme ? Ou est-elle déjà plus atomisée que la société allemande?

Jacques Rigaud : La situation en France de ce point de vue n'est pas très différente de la situation en Allemagne. Je suis frappé de voir qu'hier nous avons beaucoup insisté, avec une sorte de complaisance narcissique, sur les différences entre nos deux pays, pays fédéral, pays jacobin. Ces différences me paraissent infinitésimales par rapport aux problèmes que nous allons avoir à aborder. Et sur le fond des choses, je crois que nous allons avoir, Français et Allemands, une réflexion commune, puisque nous avons les mêmes problèmes.

Laissons un peu de côté les différences d'organisation. Je savais déjà, avant de venir ici, que j'étais un dinosaure, mais l'exposé vertigineux de Jean-Louis Missika m'en a convaincu encore plus. J'ai vécu vingt ans comme responsable exécutif de la Compagnie Luxembourgeoise de Télédiffusion, ce groupe audiovisuel européen qu'était la CLT. J'ai donc connu une époque où nous vivions à l'ombre du monopole en France, et où notre présence en Allemagne et en Belgique était extrêmement limitée. J'ai vu la rupture du monopole, la création de télévisions privées, le développement de toutes ces nouvelles technologies et l'arrivée d'Internet.

Je vais vous faire une confidence : les statuts de RTL, tels qu'ils avaient été modifiés par l'un de mes prédécesseurs, prévoyaient que la limite d'âge était de 90 ans pour le 
président, et que par conséquent, j'aurais pu être président de RTL et administrateur de la CLT jusqu'en 2022. En 1998, j'ai expliqué à mes actionnaires que, intellectuellement, j'arrivais encore à imaginer ce que pouvait être le paysage médiatique dans cinq ou dix ans, mais que je pensais qu'il était plus sage de renoncer à mes fonctions en 2000 pour donner à une génération plus jeune la possibilité de mieux affronter ces défis.

En revanche, ayant un engagement dans le domaine culturel depuis trente ans, j'ai pensé que j'avais encore des choses à faire sur le plan culturel, c'est-à-dire sur le plan institutionnel. A écouter Jean-Louis Missika, le rapport entre les êtres humains va être désormais universel ; les gens auront tous quelque chose à dire et ils auront tellement de choses à dire qu'on ne pourra pas entendre seulement le Goethe, le Hegel, le Victor Hugo, le Bergson, le Molière ou le Claudel qui naîtront dans les dix ou vingt ans qui viennent.

Si amateur que je sois de prospectives, de futurologie, et prêt à embrasser tout ce qui arrive, je considère que mon devoir de citoyen et d'homme de culture européen est de lutter contre tout ce qui, à travers ce spontanéisme protéiforme et généralisé, ferait perdre à notre civilisation son sens profond. C'est le même problème, que l'on soit Allemand ou Français. Peu importent les différences institutionnelles qui peuvent nous séparer.

Nous avons parlé hier longuement, d'une manière très intéressante, de concentration et de pluralisme. Du temps du monopole, on ne considérait pas que la concentration extrême que représentait le monopole fût un danger, ou plus exactement on avait pris quelques mesures de protection pour assurer le pluralisme. Là où je rejoins complètement ce qu'a dit Jean-Louis Missika, c'est que la révolution technologique que nous avons vécue change totalement les données du problème. Il a lui-même cité un exemple: on peut maintenant, avec toutes ces nouvelles technologies, voir ce qu'on veut, quand on veut, avec qui on veut, dans m'importe quelles conditions.

Nous sommes passés d'un monde de médias de l'offre à un monde de médias de la demande, et même plus, de l'initiative individuelle. Par conséquent, le pluralisme, que nous souhaitons tous sous l'angle de la démocratie, est du côté du consommateur. On peut assurer soi-même le pluralisme par le choix que nous faisons des différents médias auxquels nous participons.

Quant à la concentration, elle est regardée par toutes les autorités de contrôle comme un danger. Il faut la limiter, l'équilibrer, la contrôler, mais en même temps, la mondialisation fait qu' un certain nombre de produits peuvent s'imposer à l'ensemble de ceux qui sont appelés à les pratiquer ou à les consommer. On nous a cité quelques exemples terrifiants de ces sociétés nouvelles qui, en quelques années, font lire des milliards de pages par des millions de gens. La question peut se poser de savoir si nous ne devrions pas, par volontarisme, constituer ou aider à naître des groupes puissants qui, en termes d'exigence et de qualité des contenus, équilibreront cette espèce de masse informe.

Ce qui m'inquiète le plus à travers ces nouveaux médias dont je reconnais la vitalité (je trouve salubre et heureux que cela nous bouscule dans toute une série de nos habitudes) est qu'on finisse par perdre toute considération éthique et de respect de l'autre. Un exemple : il y a une grande tradition pour les journalistes, qui est le respect de ce qui est dit 'off'. Ayant reçu, en tant que président de la radio RTL, tout le personnel politique pendant vingt ans, je peux vous dire qu'aucun des propos qui ont été tenus par un ministre, un premier ministre, un homme politique, n'a été diffusé à l'extérieur. Maintenant, il y a des journalistes qui ont un blog, et notamment celui qui fait le matin à 
RTL une interview d'une personnalité qui raconte sur son blog tout ce qui s'est dit en dehors de l'émission. Je connais d'autres journalistes qui font la même chose. A l'intérieur même du journalisme, cette nouvelle technologie du blog prétend affranchir le journaliste de cette obligation élémentaire du respect de ce qui a été dit en confidence.

Les techniques et les institutions actuelles, qui sont censées garantir le pluralisme et l'équilibre des médias dans le domaine des concentrations et de la régulation, ce sont des institutions qu'il n'est pas question de supprimer. Mais les vrais problèmes qui se posent à nous en tant que société et à nos dirigeants politiques, c'est pour de donner aux jeunes générations, comme nous l'avons reçu nous-mêmes des générations antérieures, l'esprit de rigueur, de curiosité, de fréquentation des œuvres. Maintenant, on donne la parole aux auditeurs, c'est moi qui ai commencé il y a vingt ans à le faire. Maintenant, tous les jours, à tout moment, on a droit à l'opinion d'un auditeur. Je veux bien qu'il n'y ait plus de mentors, de gens qui disent d'en haut la vérité, mais il y a une médiocrité générale qui risque de résulter de ce spontanéisme qui nous pose un grave problème culturel.

Les vraies responsabilités politiques sont au niveau de l'éducation et de la viabilité de la culture institutionnelle pour équilibrer cette évolution où tout le monde devient médiateur, éditeur, où tout le monde exprime son opinion. Je crains que le lien social et, au-delà, la civilisation risquent d'être compromis.

... et la perte de l'orientation

J.-L. Missika : D'abord je voudrais respectueusement faire remarquer à Jacques Rigaud que si un Goethe ou un Molière naissait au XXI ${ }^{\mathrm{e}}$ siècle, la probabilité qu'il soit joué sur RTL TV ou sur TF1 est proche de zéro. Maintenant, je partage tout à fait son opinion ; j'ai écrit dans mon livre "La fin de la télévision » que si la question qui se posait dans le monde des anciens était de savoir qui parle, qui a le droit de parler, quelle est la légitimité qui permet la prise de parole, la question qui se pose dans le nouveau monde est plutôt de savoir qui écoute. Les gens sont tellement occupés à introduire leur propre petite œuvre audiovisuelle que la question de savoir s'ils ont également le temps de se consacrer à l'écoute des autres se pose. En gros, le problème du média puissant, prescripteur et fiable, ayant une capacité à conseiller, orienter l'auditoire vers un certain nombre d'œuvres, d'informations, d'analyses, cette question est aujourd'hui en devenir.

Simplement, les médias eux-mêmes portent une lourde responsabilité dans cette destruction des réputations. S'il y a la crise que nous connaissons aujourd'hui, c'est parce qu'il y a une désaffection du public à l'égard des médias traditionnels qui ressemble comme deux gouttes d'eau à la désaffection du public à l'égard de la politique et des institutions politiques.

Cette désaffection a quelque chose à voir avec la crise de confiance et, malheureusement, avec la connivence que vous avez décrite tout à l'heure entre les journalistes et les politiques, où évidemment, ce qui s'échangeait là ne devait pas être communiqué au grand public parce qu'il y a avait les milieux autorisés et les autres.

Alain Lancelot (dans la salle) : Je suis un septuagénaire pessimiste, mais je suis aussi un humaniste optimiste.

Septuagénaire pessimiste. Je dois reconnaître que le tableau que nous a peint JeanLouis Missika peut nous faire dresser les cheveux sur la tête parce que le «meilleur des mondes » qui se développe devant nous peut faire peur aux survivants de la planète clas- 
sique. Nous sommes tentés de penser que ce à quoi nous avons cru, ce qui nous a donné une âme et une direction pour agir est peut-être en train de se dissoudre dans cette machine à décerveler qu'est le modèle Internet. Il y a tout de même bien des raisons de penser à mon âge qu'il y a un risque de 'moyennisation' voire de médiocrité, dans cette appropriation des moyens de communication par Monsieur Tout le Monde. Je dis 'à mon âge' parce que, si j'avais 20 ans, je n'aurais certainement pas la même attitude.

\section{« Nous entrons de plain pied dans une démocratie de la communication »}

En tant qu'humaniste optimiste, je me souviens que quand la télévision a commencé à se développer, on redoutait de la voir accoucher la triste figure de "l'homme télévisionnaire » (titre d'un livre célèbre) incarnant l'anti-Goethe, l'anti-Molière, l'anti-Châteaubriand, l'anti-Dante, et ainsi de suite. Et bien non, Molière est encore lu, le nom de Châteaubriand représente encore quelque chose, comme celui de Goethe ou de Dante. Donc, je pense que ce que la télévision n'a pas détruit, Internet ne le détruira pas plus. Je crois très vraisemblable qu'une élite survivra à cette révolution technologique en lui donnant toute sa valeur, et tout son potentiel culturel.

Norbert Schneider disait tout à l'heure qu'il y aura toujours des nobles. Cela me semble très probable pour la noblesse de l'âme, et il y aura toujours des églises et des académies. Quand on regarde de près les arguments que nous pouvons brandir contre Internet, nous risquons de parler comme Burke parlait de la Révolution de France, c'està-dire que nous pensons qu'il est impossible que le peuple soit gouverné par lui-même. L'idée que l'on donne le droit de vote aux analphabètes et aux imbéciles, sans donner un droit plus important à ceux qui savent et qui sont responsables, est non seulement une absurdité mais un danger dont la civilisation chrétienne et européenne ne se remettra jamais. C'est en effet ce que M. Burke disait avec beaucoup de talent à la Chambre des Communes et il trouvait que Rousseau était un Genevois bien sot pour pouvoir prétendre que les hommes naissent libres et égaux.

On sait ce qu'il est advenu de Burke et de ses Considérations sur la révolution de France d'une part et de la démocratie d'autre part. Or nous sommes en train d'entrer de plain pied dans une démocratie de la communication, qui fait peur à ceux qui savent que le peuple a bien des défauts, qu'il peut soutenir des populismes effroyables et peut être trop facilement manœuvré. Mais l'Homme n'est jamais définitivement tombé dans des mouvements anti-démocratiques qui auraient privé la démocratie de sa valeur. Je crois que l'humanisme a encore un grand avenir devant lui, et même avec Internet plutôt que contre lui.

A. Stopp : Le politique doit-il - et si oui, comment - réagir à ces risques qui pèsent sur la démocratie ? Mais peut-être est-il trop tôt pour agir, puisque nous ne sommes pas encore capables de mesurer les effets des mutations en cours?

N. Schneider : Il y a toujours plusieurs manières de considérer l'Histoire. Nous pouvons lire les mutations des médias comme l'histoire d'un déclin, une propension très répandue chez les spécialistes des médias, et qui aboutit à une vision apocalyptique de l'évolution des paradigmes sociaux. Walter Benjamin a écrit que l'Histoire ne connaît pas le déclin. Je dirais que cela s'applique également aux médias. Les choses évoluent, nous vivons une période de transformations dans les comportements de communication, dans les nouveaux dispositifs techniques. Internet est un système grâce auquel ce qui allait lentement, était cher et rare, est devenu rapide, bon marché et disponible en 
abondance : c'est là que réside le seul changement par rapport aux autres médias. A nous de voir comment en saisir les opportunités.

J'ai mentionné la Révolution Française pour rappeler que toute tendance en appelle une autre en contrepoint. C'est cette dynamique qui fait évoluer les choses. Or actuellement, la seule chose que nous maîtrisons, c'est l'art de la prophétie. Pour une raison simple : nul ne sait si les prophètes ont raison. Il n'est pas possible de prédire les médias de demain. Ce constat me rassure en un sens, mais attise aussi ma curiosité.

Il suffit de garder à l'esprit que l'ordre a émergé du chaos pour aborder les bouleversements actuels en toute sérénité et nous poser quelques questions simples sur l'ordre à venir. Par exemple celle-ci : le Web se laisse-t-il réguler ? C'est une des questions les plus simples qui soient, mais y répondre est de la plus haute complexité. Nous nous la sommes posée. Et nous avons discuté avec Google. Avec quel résultat ? Google a retiré de son offre certaines pages susceptibles de mettre les enfants en danger. Ce ne sont que des petits pas, mais tout de même un progrès.

Un autre exemple. Nous avons appris aujourd'hui dans la Berliner Zeitung que l'annonce de la mort d'une comédienne était un fake. L'actrice est bien vivante et attend un enfant. Mais la fausse nécrologie, diffusée sur le Net, tirait sa crédibilité du fait qu'elle semblait publiée par une agence de comédiens. La tendance à la multiplication de ce genre de fausses nouvelles est pour l'instant difficile à enrayer. Car nous ne savons pas encore évaluer la crédibilité des informations circulant sur Internet. Jusqu'à présent, une information était crédible si elle paraissait dans le New York Times ou dans Le Monde, des sources de référence comme il n'en existe pas encore sur Internet.

Mais progressivement les choses s'agencent, et un nouvel ordre s'esquisse. Assurément, ces transformations s'effectuent dans une certaine opacité, mais c'est dû aussi à la numérisation qui relève du domaine de l'abstrait, de la promesse de mondes virtuels.

\section{Considérer la société de l'information non pas sous l'angle d'une régulation,} mais sous celui d'un rééquilibrage mondial entre Europe et Etats-Unis

René Lasserre (dans la salle) : Nous avons beaucoup parlé de la Toile universelle mais j'aimerais que nous en venions à notre 'Toile européenne', nous Français et Allemands. Ne peut-on pas penser que ce décloisonnement de l'information et de l'échange culturel peut être aussi une chance pour l'Europe ? Une chance de surmonter cette fragmentation des identités nationales qui sont cimentées en quelque sorte par nos systèmes de radiotélévision institutionnels nationaux ? Je crois que s'il y a un obstacle à l'émergence d'une opinion publique européenne, c'est bien là qu'il faut le chercher.

Est-ce que justement, il n'y a pas aujourd'hui une chance d'européaniser la société par le bas, de faire émerger une société européenne chez les nouvelles générations, par de nouvelles techniques, un nouveau rapport à l'information et la communication ? Cela pose aussi la nécessité d'une régulation, européenne cette fois-ci, de ces nouveaux médias, dans la mesure où cela peut être possible. Peut-être qu'à l'échelle européenne, cela a plus de sens qu'à l'échelle purement nationale ?

J.-L. Missika : Je vais répondre indirectement à la question, pour revenir sur cet aspect culturel qui a fait débat. Il y a des choses très positives dans Internet au plan culturel. Je vais citer un exemple qui m'a beaucoup frappé. C'est ce que les Américains appellent la théorie de la "long tail », de la longue file d'attente, c'est le constat que lors- 
qu'il y a la possibilité d'avoir accès à un catalogue important d'ouvrages : livres, disques, films, les gens choisissent de façon beaucoup plus diversifiée que quand ils n'ont accès qu'à des catalogues plus limités. Aujourd'hui, dans les librairies, on ne vend que des livres sortis depuis moins de six mois ou qui sont des best-sellers. On se rend compte sur Amazon ou sur d'autres sites de vente de livres que $50 \%$ des commandes passées par les internautes concernent des ouvrages qui ne sont pas disponibles en librairie. C'est aussi vrai pour la musique et le cinéma. Il y a un site américain, Netflix, qui propose 60000 films à louer. Les commandes quotidiennes de catalogue sont de 40000 films différents.

On a beaucoup parlé hier de diversité culturelle et de pluralisme, on se rend compte qu'Internet est une immense machine à produire de la diversité culturelle et du pluralisme. Je me situe résolument du côté des optimistes humanistes, comme Alain Lancelot.

Maintenant, sur l'Europe, je serais plus nuancé, car les jeunes générations sont mondiales, elles ont une approche globale. Les jeunes vont indifféremment sur YouTube, qui est américain, et sur Délimotion, qui est l'équivalent français de YouTube. Ils vont indifféremment sur My Space et sur Sky Blog. Ce qui fait sens est la langue : il est clair qu'il y a des communautés virtuelles francophones, comme il doit y avoir des communautés germanophones ou hispanophones qui se constituent sur le Web. La langue est un facteur de construction de la communauté virtuelle. Mais l'autre langue pratiquée est l'anglais. De ce point de vue, on est face au même obstacle qui était le nôtre dans l'ancien monde.

Sur la régulation européenne, mon sentiment est le suivant: aujourd'hui, ce qui manque à l'Europe, c'est de parler d'une seule voix à l'OMC sur ces questions de propriété intellectuelle et d'abus potentiels de position dominante de sociétés comme Google, MSN ou Yahoo. Le problème majeur ne me semble pas un problème de régulation, mais de réussir à faire en sorte que la question de la société de l'information devienne une question centrale, soit à l'OMC, soit dans d'autres structures, éventuellement mondiales, où l'on puisse débattre notamment de la question du rééquilibrage entre les Etats-Unis et l'Europe. Maintenant, si vous me demandez pourquoi toutes les sociétés que j'ai citées sont américaines et qu'aucune n'est française ou allemande ou européenne, la réponse est très simple : le malthusianisme a des effets catastrophiques sur la capacité des grands groupes européens à devenir des acteurs mondiaux de la nouvelle donne de l'information.

Jürgen Heinrich (dans la salle) : Je ne suis pas vraiment d'accord avec la description et le jugement de valeur implicite qui l'accompagne. Car si on considère les études sur la consommation des médias, on remarque un allongement de la durée passée à regarder la télévision, à écouter la radio, conjugué au fait qu'un auditeur n'écoute que 1,6 radio et qu'un téléspectateur passe près de $60 \%$ de son temps à ne regarder que 2 chaînes. La fragmentation de la demande n'est donc visiblement pas si importante que cela.

Mais je suis encore moins d'accord avec le jugement de valeur implicite. Les émissions à très grande écoute, donc fédératrices, sont présentées comme un idéal. Or du point de vue de l'économiste, c'est plutôt une vision d'horreur - tout à l'inverse de la situation actuelle avec son offre très diversifiée, qui est un paradis.

J. Rigaud : Sur l'audience, j'ai entendu très souvent cet argument qu'on m'a opposé par rapport à ce que j'ai pu écrire. La réponse est extrêmement simple : la mesure d'audience de la télévision est un consensus de marché (ce n'est pas une objectivité 
scientifique) : les acteurs principaux de l'audiovisuel se réunissent et se mettent d'accord pour la définition de la monnaie unique qui est le point d'audience de la télévision.

Aujourd'hui, la télévision sur ADSL en France comme en Allemagne n'est pas mesurée, il n'y a pas de mesure multimédia, c'est-à-dire qu'on ne tient pas compte de ce qu'on appelle la consommation cross-média. Aujourd'hui, vous entrez dans la chambre d'un adolescent : la télévision est allumée, mais il est devant son ordinateur. Il 'chatte', il envoie des SMS sur son téléphone, il fait ses devoirs en même temps et quand le téléphone sonne, il répond. Il consomme quatre médias en même temps qu'il fait ses devoirs. Vu du point de vue de la mesure d'audience de la télévision, il regarde la télévision, mais de quoi parle-t-on? Voilà ma réponse.

« Nous savons gérer la pénurie, mais nous n'avons pas appris à gérer l'abondance »

N. Schneider : Jürgen Heinrich a souligné le fait qu'on puisse se demander pourquoi la consommation n'a pas changé de manière significative alors que l'offre a augmenté sans commune mesure. Les chiffres évoqués reflètent au fond le réflexe qu'a le consommateur de ne consulter que ce qu'il connaît ; il n'a pas vraiment idée de ce qu'il pourrait avoir s'il le voulait.

Face à cette abondance, il conviendrait plutôt de se demander s'il ne pourrait pas y avoir une meilleure source d'orientation ou d'information que celles que préconisent les défenseurs des émissions grand public. Qu'avons-nous à notre disposition ? Comment pouvons-nous y accéder? Qu'est ce que cela nous apporte?

Or pour des raisons liées à l'histoire de nos cultures, nous ne nous posons jamais ces questions. Nous avons appris au fil des siècles à gérer la pénurie. Mais nous n'avons pas appris à gérer l'abondance, du moins en ce qui concerne les médias. Voilà la problématique que nous devrions aborder, dans une perspective générale comme sous l'angle de l'organisation adéquate de cette abondance.

Qui va régner sur l'offre ? Pour le dire de manière populiste : un hedge fund, guidé par la seule logique du profit ? Son approche ou celle d'un autre acteur sera-t-elle purement économique, à quoi il n'y aurait rien à redire en soi, ou alors suivra-t-il une approche culturelle qui voudrait par exemple que tout ce qui est européen mérite d'être particulièrement mis en valeur ? Qui nous guidera alors à travers ces musées de la mémoire informatique pour nous montrer aussi bien les Goethe ancienne que nouvelle manière ? Qui nous permettra de nous orienter dans la jungle des blogs? Qui nous pilotera dans ce nouveau monde à l'instar d'un GPS ?

A. Stopp : Pourra-t-on encore à l'avenir contrôler ceux qui contrôlent les médias ?

N. Schneider : Il est impossible de contrôler avec les mêmes instruments quelque chose qui aura changé. Nous pensons jusqu'ici qu'on ne peut réguler que si on peut également contrôler et qu'on ne peut contrôler que si on peut sanctionner. C'est une façon de voir les choses que nous devrons abandonner à l'avenir. Cela ne signifie pas remettre en question la régulation en tant que telle, mais elle devra trouver de nouveaux instrument. Il est simpliste de ne raisonner qu'en termes d'interdiction et d'autorisation, car il y a un vaste monde des possibles entre ces deux extrêmes.

Le développement d'Internet montre que nous devrions accorder une plus grande importance aux accords internationaux et les développer. C'est un processus long et difficile mais qui ouvre des perspectives, à l'instar de l'évolution de l'ONU qui, aussi 
problématique qu'elle soit par ailleurs, n'en porte pas moins ses fruits. Pourquoi n'en irait-il pas de même pour d'autres problématiques du monde globalisé ?

Une chose est certaine : conserver le mode de régulation établi, à l'échelle nationale, n'est pas la solution. Si la semaine prochaine, ce n'était pas Berlusconi qui souhaitait racheter ProSiebenSat.1 mais un fonds canadien, ce dernier aurait le droit de le faire, car il n'est pas encore présent sur le marché allemand. Aucune régulation particulière n'est nécessaire. Mais si ce même fonds possède une participation en Suède, et que cette société suédoise voulait en racheter une autre en Allemagne, alors ce cas ne dépend plus du droit national, bien qu'il pose un problème du point de vue du droit de la concurrence. Il faut régler ce problème à un autre niveau au lieu de nier le problème, comme nous avons encore trop tendance à le faire. Cette attitude est totalement improductive.

«Considérer plutôt comment l'ensemble des médias peut nous conduire à la culture réelle »

J. Rigaud : Je voudrais revenir sur la question des jugements de valeur implicites qu'a posée Jürgen Heinrich et à laquelle je n'ai pas répondu. C'est vrai que l'époque où les rues étaient vides parce que toute une ville, tout un pays était devant une émission de télévision est révolue. Tant mieux d'ailleurs, parce que ça montre la diversité du choix et l'autonomie des choix individuels. Néanmoins, il existe un certain nombre d'événements qui sont de nature à fédérer toute une nation et même l'Europe. On l'a vu dans le domaine du sport, avec le Mondial de football. Les mesures d'audience ne m'intéressent plus : je ne suis plus dans une chaîne de télévision ou de radio, donc c'est un problème de gestion, de rentabilité, il est économiquement important de savoir quel est le potentiel d'une émission ou d'une autre.

Ce qui m'intéresse sur le plan social et culturel, c'est l'impact d'un événement, d'une œuvre, d'une question à travers ce que l'ensemble des médias en disent. La guerre d'Irak, ce qui s'est passé au Liban, à travers l'ensemble des médias, des chaînes de télévision, même les moins portées sur l'information, a donné à chacun conscience de ce qui se passait. Ce qui s'est passé aux Etats-Unis en septembre 2001, ces images d'avions percutant les tours, des milliards d'êtres humains ont ces images gravées dans leur esprit à travers l'ensemble des médias auxquels ils ont pu faire référence. Je suis très frappé sur un plan proprement culturel de voir combien actuellement, du fait de l'information même très limitée, très superficielle que peuvent donner les chaînes de télévision, même les moins portées sur la culture ; de l'ouverture par exemple d'un musée comme le musée du Quai Branly et la fréquentation que cela induit.

Ce qui m'intéresse, c'est l'impact de l'ensemble des médias, Internet compris, et la manière dont l'ensemble des médias peut nous conduire à la culture réelle. Je ne suis pas du tout un ennemi d'Internet, je suis plutôt excité par l'ensemble des potentialités.

La seule chose que je redoute, c'est que les jeunes générations finissent par vivre uniquement dans le virtuel et que disparaisse ce que nous avons tous appris depuis les origines de l'Homme sur le caractère sensuel du rapport à la culture, à l'événement, à l'œuvre. Expliquez-moi pourquoi l'émotion que vous éprouverez quand vous serez devant cette œuvre, là où elle a été peinte, cette émotion et la mémorisation n'est en rien comparable à ce que l'image télévisée la plus perfectionnée vous donnera.

Ce qui me réconforte dans l'attitude des jeunes, actuelle, qui sont submergés d'informations et de données par Internet, c'est qu'ils ont encore le goût de la ferveur en 
commun et que si tel chanteur se produit, il y aura 50000 jeunes qui iront, parce qu'ils seront ensemble avec leur briquet allumé et qu'ils vivront ensemble autre chose que ce que chacun peut vivre dans sa chambre à travers la culture média. Je crois que notre responsabilité est de maintenir l'offre d'une culture vécue par rapport à l'immensité de la culture virtuelle.

Tout ce qu'on pourra faire en matière de régulation est sans aucune espèce d'intérêt, ni de portée, parce que le problème est mondial, parce que la régulation sera toujours en retard. En revanche, il importe que nous parvenions, nous autres Européens, à donner par l'école, par l'éducation, le sens d'un certain nombre de valeurs, d'une exigence qui nous fera refuser le mensonge, la contre-vérité, la désinformation. C'est la même idée que la démocratie d'après Burke ; c'est vrai, c'était extrêmement dangereux de donner le droit de votre aux imbéciles ou aux demeurés... Et en même temps, tout l'effort de l'instruction et de l'éducation a fait que la plupart de ceux qui contribuent au vote le font d'une manière humainement digne.

" Entre le moment où une technologie se déploie et le moment où des

pratiques sociales apparaissent, il s'écoule plusieurs dizaines d'années »

J.-L. Missika : Je partage beaucoup de ce qu'a dit Jacques Rigaud mais comme par définition un débat est un jeu de rôles, je vais prendre la défense de la génération numérique.

D'abord je voudrais faire remarquer qu'on en est encore au début du déploiement d'Internet en tant que pratique sociale. Par définition, cela va prendre peut-être 40 ou 50 ans pour que l'ensemble des usages sociaux et culturels soient inventés et stabilisés. On parle de choses embryonnaires.

Il faut être très prudent dans les analyses et les prédictions, parce que si on se reporte 40 ans en arrière et qu'on regarde ce qu'était la télévision, on apprend à relativiser : il n'y avait alors qu'une seule chaîne, et beaucoup de sociologues ont écrit beaucoup de sottises sur la télévision comme média totalitaire, ayant une influence politique excessive et faisant l'élection par exemple, et ainsi de suite. Entre le moment où la télévision a commencé et le moment où, à moins avis, elle arrive un peu à sa fin, le média n'a plus rien à voir avec ce qu'il était. Or aujourd'hui, Internet en tant que média est en devenir.

$\mathrm{Si}$ je continue cette analogie encore plus loin dans le temps, jusqu'en 1830, je dirais qu'au moment où est née la presse généraliste populaire : la penny press, aux Etats-Unis et en Europe, on n'avait pas encore inventé le modèle économique qui permettait de financer la production d'information, sa diffusion et de payer des journalistes professionnels. La presse d'avant 1830 était faite par des amateurs : des avocats, des bourgeois, des notables, et la professionnalisation de l'information s'est faite progressivement entre 1830 et 1850, alors même que la rotative avait été inventée bien avant.

Entre le moment où une technologie se déploie, le moment où des pratiques sociales apparaissent, le moment où elles sont 'monétisables', c'est-à-dire où on peut gagner de l'argent avec parce que des entrepreneurs schumpétériens se disent : je vais prendre des petites annonces, de la publicité, du paiement à l'acte, etc., il se passe plusieurs dizaines d'années. C'est exactement ce qu'on est en train de vivre aujourd'hui. La percée conceptuelle permettant, sur Internet, de concevoir des produits d'information, fabriqués par des professionnels et viables économiquement sur le long terme, cette percée con- 
ceptuelle n'a pas encore eu lieu, mais je considère qu' elle devrait avoir lieu dans les dix ans qui viennent.

Isabelle Bourgeois (dans la salle) : Dans le prolongement de ce que vient de dire Jean-Louis Missika, on ne peut pas assez souligner le fait que l'émergence du modèle économique de la presse dans la première moitié du $\mathrm{XIX}^{\mathrm{e}}$ siècle s'est accompagnée de la standardisation de l'information. Les nouvelles technologies - en l'occurrence, le télégraphe - avaient permis de concevoir de nouveaux produits : la dépêche. Elles ont déterminé les règles applicables aux contenus, ou plus exactement à leur présentation, autrement dit : à la standardisation de l'information. Or ce sont ces règles qui font que l'information a un sens. Au même moment, dans un contexte d'effervescence comparable à celui que nous vivons actuellement, se constituait un système de référence propre à cette ère, avec ses offreurs et ses agrégateurs de contenus.

L'histoire des médias nous apprend autre chose encore. Plusieurs intervenants ont évoqué ce droit de vote accordé aux 'idiots'. Le système éducatif a fait en sorte que l'individu, cet 'idiot' à l'origine, est devenu un citoyen conscient et responsable. Les médias de masse y ont contribué eux aussi. Et je vais prendre ici comme exemple le livre, dont on oublie trop souvent qu'il n'est devenu un média de masse que depuis une petite cinquantaine d'années. Auparavant, il était un objet de luxe pour une élite restreinte. Certes, au XXe siècle, plus de personnes savaient lire que du temps de Gutenberg.

Si l'alphabétisation a été la première étape vers la massification de la lecture, l'étape décisive n'a été franchie qu'avec l'apparition du livre de poche, produit en masse, vendu à bas prix, et distribué partout. Cette massification a mené à une réelle démocratisation - une révolution qui n'a pas détruit notre société, bien au contraire. Car dans le même temps, les compétences de base, dont la maîtrise de la lecture, se massifiaient aussi. Norbert Schneider a souligné tout à l'heure le fait que nous n'avons pas encore appris à vivre avec l'abondance que nous connaissons actuellement. La même problématique n'a cessé de se poser depuis le milieu du XIXe siècle. Voilà le premier enseignement que nous permet de tirer l'exemple, récent, de la massification du livre.

\section{" On ne peut pas contrôler ce qui est en mutation »...}

Le second est une interrogation. Au lieu de chercher désespérément à figer les choses en l'état, c'est-à-dire à mettre en place une régulation, tâche impossible au demeurant dans l'univers d'Internet, ne devrions-nous pas nous demander plutôt ceci : et si la clef résidait dans une alphabétisation nouvelle manière ? Lors de la structuration du lien entre médias et société depuis le XIXe, elle a joué un rôle déterminant, apportant et développant chez le consommateur des médias, donc au niveau de la demande, les compétences nécessaires à la formation de l'opinion, permettant à chaque individu de se repérer au sein d'une offre abondante et diverse, et de procéder à des choix.

Nous avons appris à lire, à écrire, à compter. Et nos compétences ont évolué au fil du temps, s'adaptant à un environnement lui-même évolutif. N'est-ce pas là le mécanisme clé du lien social ? Notre approche du nouvel univers d'abondance dont le pivot est désormais la demande et non plus l'offre ne devrait-elle donc pas plutôt être dynamique?

Autrement dit, élargir les compétences du citoyen, lui donner un système de lecture et de valeurs lui permettant de se repérer dans cette offre pléthorique - n'est-ce pas là un 
moyen plus adéquat pour répondre aux défis ? Le contrôle se déplacerait ainsi de l'offre vers la demande, son outil de la régulation vers la diffusion de compétences. Reste la question de savoir quelles institutions ou quels réseaux dispenseraient ce nouveau savoir actif du citoyen...

N. Schneider : Cette discussion sur les nouveaux et les anciens médias ne date en effet pas d'hier. L'apparition de l'écriture a été fortement critiquée par Platon. Pourquoi ? Une des raisons était qu'on ne peut pas contrôler ce qui est en mutation. Cela signifie, si on cherche une analogie métaphorique avec la situation que nous vivons aujourd'hui, que l'autorité et la portée des paroles des papes de la prédiction qui nous ont expliqué la vie dans le monde de l'analogique est aujourd'hui remise en question. C'est une situation typique, et il s'agit maintenant de savoir où se situent les nouvelles instances de prédictions et de définition des doctrines.

\section{La seule régulation possible est « une éducation à la compréhension du monde »}

Et si on va jusqu'à dire que l'éducation en tant que sous partie de la formation est aussi une forme de régulation (et je ne pense pas qu'il existe de plus forte régulation que l'éducation), alors la carte de la formation est l'une des plus importantes qu'on doive jouer. Il ne s'agit pas seulement de la question de la compétence face aux médias, mais de bien plus encore: d'une éducation à la compréhension du monde, qui passe par l'apprentissage de la distinction entre le réel et le message sur le réel, entre ce qu'il vit et ce qu'il voit, qui confère au citoyen le recul nécessaire pour faire ses choix. C'est là l'élément culturel qui doit être intégré dans nos réflexions sur notre nouvelle approche du contrôle des médias. Et il commence à l'être, du moins en Allemagne. 


\section{Debat $\mathbf{N}^{\circ} 5$}

\section{LES ENTREPRISES S'EUROPEANISENT.}

Faut-il un contrôle des concentrations pour les médias à l'échelon européen ? 



\title{
DEBAT $N^{\circ} 5$
}

\section{LES ENTREPRISES S'EUROPEANISENT. Faut-il un contrôle des concentrations pour les médias à l'échelon européen ?}

\author{
EXPOSE INTRODUCTIF : Thomas Kleist (EMR) \\ INTERVENANTS : \\ Olaf Christiansen (Bertelsmann AG) \\ Peter Eberl (DG Concurrence, Commission européenne) \\ Werner Schwaderlapp (me :mi, Europe FH) \\ Débat animé par Joseph Lorent (Luxemburger Wort)
}

\begin{abstract}
Les règlementations nationales sont mises au défi par des mutations qui concernent l'espace communautaire et, par-delà, le marché mondial. Quel peut être l'échelon le plus adéquat pour prendre en considération cette nouvelle donne?

A l'heure actuelle, les phénomènes de concentration observés au niveau des offreurs de services se cantonnent certes encore dans les frontières nationales. Et il n'existe pas, dans le secteur des médias, d'entreprises qui soient réellement de taille européenne. Mais l'articulation existante entre le contrôle par la Commission des positions dominantes sur le marché de l'UE et le contrôle sectoriel des concentrations par les autorités nationales résistera-t-elle aux mutations qui s'esquissent?

Certes, les groupes présents dans plusieurs Etats membres ne mettent guère encore en réseau leurs activités sur les différents territoires, et n'ont pas non plus développé de stratégie unique. La production des contenus n'est pas européanisée; la diversité culturelle et linguistique s'y oppose. De ce fait, rien ne légitime (pour l'instant?) une régulation sectorielle des médias à l'échelon communautaire. Mais qu'en est-il de certains segments de marché, comme celui de la distribution des droits de diffusion, fortement internationalisés ?

Comment considérer également le segment des plateformes (comme réseaux de distribution et prestataires de contenus) à qui revient un rôle croissant de gate keepers ? Ce segment ne constitue pas seulement un goulot d'étranglement. Dans la mesure où il structure l'offre, il influe également sur la demande ; et en proposant une orientation dans une offre pléthorique, il aura de même une influence sur l'opinion.

Certes, l'opinion publique européenne n'existe pas ; la question du pluralisme de l'offre ne se pose donc pas à l'échelon communautaire. Certes aussi, au regard du droit de la concurrence, c'est la demande qui détermine la concentration des acteurs; or cette demande n'est pas européenne, mais nationale. Rien ne semble donc légitimer une régulation sectorielle des médias à l'échelon communautaire. Et pourtant, celle-ci est souvent revendiquée...
\end{abstract}





\section{EXPOSE INTRODUCTIF}

\section{En l'absence d'un espace public européen, l'échelon national reste le cadre pertinent de la garantie du pluralisme}

\section{Thomas Kleist}

La vitesse du changement que nous observons, particulièrement dans le domaine de la communication, amène à se demander si les législateurs sont encore à même de traduire dans la réalité cet "ordre positif» que le Tribunal constitutionnel fédéral leur avait demandé de mettre en œuvre. Il entendait par là un modèle de régulation qui garantirait que toute la diversité des opinions puisse s'exprimer dans l'audiovisuel, que ce pluralisme mène à une information exhaustive et que l'audiovisuel soit ainsi indépendant à la fois de l'Etat et d'un groupe prédominant dans la société.

C'est ce qui m'a amené à me demander ce que recouvre ce «nous » qui figure dans l'intitulé de cette table ronde : "Avons-nous besoin d'un contrôle des concentrations au niveau européen? ». Faut-il entendre par là les entreprises qui veulent s'étendre librement et sans restriction dans toute l'Europe dans le but d'accroître leur valorisation boursière ? Ou ce «nous » veut-il dire le peuple, cette communauté de citoyens où, conformément à l'article 5 de la Loi fondamentale, "chacun a le droit d'exprimer et de diffuser librement son opinion... et de s'informer sans entraves aux sources qui sont accessibles à tous »?

Ou, pour formuler ma question autrement : s'agit-il de protéger les mécanismes du marché, s'agit-il de garantir le pluralisme ou s'agit-il des deux à la fois, ces deux approches constituant les deux faces d'une même médaille?

Nous avons donc affaire à une situation relativement classique où des motivations diverses mènent à la poursuite d'objectifs de même nature. A l'échelon communautaire ont été adoptées des règles régissant la concurrence en général. Or à l'échelle nationale n'existent jusqu'ici que des réglementations sectorielles, spécifiques à la concentration dans le domaine des médias. Et comme toute réglementation communautaire doit être justifiée en raison des principes de subsidiarité et des compétences d'attribution, la question qui se pose à nous est dès lors celle-ci : y a-t-il des raisons irréfragables, existantes ou prévisibles, pour créer des règles communautaires - ou tout du moins des règles harmonisées au sein de l'Union - visant à garantir le pluralisme des opinions et pour faire contrôler également leur application par une autorité européenne ?

Norbert Schneider, président de la Landesmedienanstalt de Rhénanie du NordWestphalie, considérant que l'analyse des marchés des médias d'un point de vue purement national est de plus en plus discutable, estime que désormais, la donne européenne doit être prise en compte. Et de fait, on peut se demander si l'européanisation ou la globalisation des groupes médiatiques n'exige pas en retour une réponse communautaire en termes de garantie du pluralisme. 


\section{L'expansion économique des entreprises}

Les conditions cadre pour les entreprises en expansion se sont considérablement améliorées au cours de ces dernières décennies. L'engagement sur d'autres marchés, surtout dans les autres Etats membres de l'Union Européenne, a été facilité par la mise en œuvre conséquente de la doctrine du marché commun. Songez par exemple aux anciennes entreprises publiques dans le secteur des télécommunications qui ont d'abord étendu leurs antennes vers les opérateurs de téléphonie fixe dans les Etats en voie de démocratisation en Europe centrale et orientale, pour conquérir ensuite les marchés de la téléphonie mobile grâce à l'obtention de licences ou au rachat d'opérateurs existants. Ces mêmes entreprises de télécommunications fixes et mobiles cherchent aujourd'hui à se développer sur les marchés d'Europe de l'ouest grâce à la commercialisation de contenus audiovisuels sur leurs réseaux mobiles ou, via des protocoles IP, sur leurs réseaux fixes.

Ces tendances sont connues, de même que les bouleversements subis par les éditeurs de presse. On a pu s'apercevoir récemment, ici à Berlin, combien les groupes allemands sont aux prises de ces mutations générales, que celles-ci peuvent révéler l'étroitesse des liens qu'entretiennent la presse et le politique, plaçant ce dernier au centre d'âpres débats comme lors de la révision du droit des fusions spécifique de la presse écrite dans le cadre de la réforme de la Loi contre les entraves à la concurrence (Gesetz gegen Wettbewerbsbeschränkungen, GWB).

Le processus de concentration à l'œuvre dans de nombreux pays européens sur les marchés du câble et de l'accès à Internet est un exemple de plus de ces bouleversements. Il nous a contraints à nous interroger sur la manière de prendre en considération les investisseurs financiers (décrits comme des «sauterelles ») dans la régulation des médias. C'est ce qu'a fait Victor Henle, président de la Landesmedienanstalt de Thuringe. Dans un article très remarqué publié dans la revue epd medien, il est parvenu entre autres à la conclusion que le développement des sociétés de participations financières va rendre considérablement plus difficile le contrôle de la concentration des médias.

La question se pose avec une acuité particulière dans le segment de l'audiovisuel où le capital d'au moins une des grandes «familles » du duopole de la télévision privée (le groupe ProSiebenSat.1) est aux mains d'investisseurs, et l'y restera probablement à l'avenir. Pour ce qui est de l'autre «famille» (RTL Group où est impliqué Bertelsmann), il s'agit toujours d'un groupe de dimension internationale qui poursuit une stratégie de développement forcé en Europe centrale et en Europe de l'est. Il s'y heurte à la concurrence d'entreprises pour la plupart américaines qui ont essaimé en Europe, comme SBS, CME, ou à celles des Murdoch et Malone.

Ces quelques éléments mettent en lumière le développement mondial du secteur des médias, son intégration globale, et confirment ainsi le point de départ de nos réflexions.

\section{Le cadre juridique européen : libertés fondamentales...}

J'en viens ainsi aux conditions-cadre juridiques. Considérons pour commencer le niveau européen et, à cet échelon, par ce qu'il est convenu d'appeler les libertés fondamentales, celles-là mêmes qui favorisent les activités transfrontières des groupes médiatiques.

Il s'agit d'une part de la libre circulation des marchandises (art. 28 du Traité CE) qui joue un rôle considérable pour les médias offline comme les quotidiens, les périodiques, la musique sur CD et les films sur DVD. D'autre part, de la liberté d'établissement, de 
la libre circulation des capitaux et des services (art. 43 sq., art. 56 sq. et art. 49 sq. du Traité $\mathrm{CE}$ ). Les deux premiers principes évoqués permettent à une entreprise soit de s'installer directement sur le marché d'un autre Etat membre en y créant filiales ou succursales, soit d'y pénétrer via l'acquisition de parts de capital dans des entreprises existantes. Quant à la libre circulation des services, elle permet la diffusion transfrontières des émissions audiovisuelles et autres services de la société de l'information.

L'application de ces libertés aux activités citées en exemple ne souffre aucune limitation au sein de l'espace communautaire, même pour des raisons purement économiques. Des règles spécifiques discriminatoires, c'est-à-dire appliquées aux ressortissants d'un autre Etat membre ou limitant l'implantation d'une entreprise de nationalité étrangère doivent être toujours être justifiées et ne sont licites que dans un nombre de cas très limité, par exemple la sécurité publique. Les Etats membres peuvent certes prendre, au nom de l'intérêt général, des mesures non discriminatoires élevant des barrières à l'entrée d'un acteur issu d'un autre Etat membre ; mais la pertinence de ces dernières est minutieusement contrôlée par la Commission européenne et la Cour européenne de justice. En outre, de telles mesures d'exception ne trouvent plus leur application que dans les rares domaines qui échappent à l'harmonisation des règles au sein de l'UE. La Directive TSF apporte une telle harmonisation du cadre réglementaire communautaire pour les activités médiatiques.

Rappelons aussi que les différences de réglementation entre les Etats membres, desquelles peuvent découler des entraves aux libertés éditées par le marché commun et qui, pour cette raison, font l'objet de mesures d'harmonisation au sein de l'UE, sont généralement le point de départ d'initiatives prises par la Commission, relayées ensuite par le Parlement et le Conseil. Ajoutons à cela que, en ce qui concerne la liberté d'établissement et la libre circulation des services, le Traité des Communautés Européennes a confié au législateur de l'UE la mission exprès de prendre toute disposition afin de faciliter l'exercice effectif de ces libertés (art. 47 Traité CE). Cet impératif d'adaptation mène souvent à une harmonisation selon le plus petit dénominateur commun, d'un côté, et de l'autre, il fait que les marges de manœuvre des Etats membres deviennent de plus en plus étroites.

\section{... et droit de la concurrence}

Les dispositions du droit de la concurrence constituent un deuxième pilier du cadre juridique communautaire : l'interdiction des ententes (art. 81 Traité CE) ainsi que de l'abus de position dominante (art. 82), et le contrôle des fusions (Règlement sur le contrôle des concentrations). S'ajoutent à ce cadre réglementaire les pouvoirs de contrôle de la Commission lorsque des droits exclusifs ou particuliers sont accordés à certaines entreprises (c'est là le point de départ de la régulation sectorielle des marchés des télécommunications), et plus généralement dans le cas des services d'intérêt économique général (art. 86 Traité CE) ; elle est également compétente en ce qui concerne les aides d'Etat (art. 87).

L'approche du droit de la concurrence au sens étroit du terme est toujours fondée en premier lieu sur la définition du marché pertinent. C'est ensuite seulement qu'est déterminé si l'un des acteurs ou un groupement d'acteurs sont en position dominante sur ce marché. Le contrôle des abus implique qu'on se demande en outre si des avantages 
peuvent être tirés de cette position dominante au détriment des concurrents, puis du développement du marché dans son ensemble. En ce qui concerne le contrôle des fusions, la question centrale est de savoir si de cette fusion va naître ou se renforcer une position dominante sur le marché.

Le droit de la concurrence régule donc la dimension économique sous l'angle de l'offre, afin de garantir un fonctionnement sans entraves de la concurrence. Il est intéressant de noter ici que dans le Règlement sur le contrôle des fusions figure une disposition qui soustrait à son domaine d'application certaines décisions de régulation des marchés prises par les Etats membres dans des contextes et pour des motifs particuliers ; c'est la cas, en l'occurrence, du pluralisme des médias (art. 21 \$2 Traité CE). On estime donc, du point de vue de la théorie du droit, qu'il est possible que la Commission autorise une fusion mais que l'autorité régulant les médias au niveau national prenne une toute autre décision pour des raisons de protection du pluralisme.

\section{Les cadres juridiques nationaux}

Contrairement à ce qu'on observe au niveau communautaire, les Etats membres se caractérisent généralement par la juxtaposition du droit de la concurrence et d'un droit des concentrations particulier aux médias. En ce qui concerne ce dernier, il y a unanimité pour considérer qu'il poursuit des objectifs différents du droit de la concurrence et qu'il n'est donc pas envisageable de renoncer à une régulation spécifique ou de lui substituer la conception purement économique du droit des cartels.

Le droit de la concentration des médias varie considérablement d'un Etat membre à l'autre. On observe néanmoins certaines similitudes : la plupart du temps, il repose soit sur la prise en considération des participations détenues et des parts de capital (comme c'est le cas en France) soit sur celle de la part détenue sur le "marché de l'audience » (Allemagne). On peut aussi trouver des modèles qui combinent les deux approches. En Italie par exemple, le droit des médias, réformé en 2004, prend en considération (mais pour combien de temps ?) le marché de la communication dans son ensemble ; le critère retenu est dès lors un seuil de chiffre d'affaires.

Les réglementations nationales divergent radicalement en revanche par leur approche de la manière dont il convient d'évaluer le potentiel d'influence pluri-médias dans le cas d'activités intégrant les divers segments du marché médiatique, au niveau horizontal, mais aussi vertical.

En Norvège et aux Pays-Bas, on suit en outre une approche consistant à créer plus de transparence sur les marchés des médias grâce à une analyse continuelle des participations et de liens capitalistiques entre groupes de médias. Cela s'effectue en partie au travers des registres rendus publics et en partie par la publication de rapports annuels afférents. Le travail de la KEK allemande est lui aussi déterminé par le recensement et la publication du degré d'interpénétration des acteurs des médias.

La comparaison des régimes de régulation des médias en Europe fait apparaître deux traits communs : l'objectif est toujours de garantir la pluralité des médias via la protection contre une opinion dominante; dans la plupart des Etats, cette mission de protection a été confiée par la Constitution ou la jurisprudence constitutionnelle aux législateurs qui doivent adopter les dispositions nécessaires. Ensuite, ces dispositions avant tout de nature structurelle, et qui visent à garantir la liberté d'opinion en général, suivent 
une approche commune : elles visent explicitement à préserver la pluralité de l'offre et, implicitement, à limiter la concentration économique des opérateurs.

Par ailleurs, il existe une multitude d'autres mesures de régulation visant elles aussi à la protection du pluralisme : la préservation de l'audiovisuel public, les règles du must-carry et du must-offer, les obligations de programmes (par exemple en ce qui concerne les œuvres de producteurs européens ou indépendants, les quotas linguistiques, diverses obligations particulières comme la programmation d'émissions régionales ou d'émissions produites par un tiers, en Allemagne, ou celles découlant, en France, de l'obtention de la licence), la garantie d'un accès non-discriminatoire, équitable et ouvert à tous, la promotion des œuvres cinématographiques, et ainsi de suite.

\section{Préserver au sein de l'UE l'hétérogénéité des mesures nationales}

Ce bref inventaire révèle toute la complexité de la situation. Les mesures prises à l'échelon communautaire se concentrent sur les aspects économiques des médias : elles régulent des services et protègent le jeu de la libre concurrence contre les risques de distorsion découlant de positions dominantes. Les mesures prises à l'échelon national sont mues par des objectifs démocratiques et de politique culturelle. Jusqu'ici, les principes de subsidiarité et des compétences d'attribution interdisent à l'échelon communautaire d'adopter une réglementation spécifique aux médias et visant la garantie du pluralisme.

Ainsi se pose en permanence la question de savoir si le processus de développement du cadre réglementaire de l'UE exige ou non la mise en place de règles homogènes en vue de la protection du pluralisme et, le cas échéant, l'institution d'une autorité de régulation spécifique.

A mon sens, aucune raison contraignante ne l'impose - du moins, pas pour l'instant. Depuis 15 ans que je défends cette thèse, elle semble concorder avec la position officielle de «Bruxelles », même si on a pu constater à intervalles réguliers la tentation de l'échelon communautaire de se saisir de cette question. Estimant que les diverses mesures nationales de protection du pluralisme constituaient une entrave au bon fonctionnement du marché intérieur, le Parlement Européen avait par exemple réclamé, dans une résolution de 1994 qui se fondait sur le «Livre vert sur le pluralisme et la concentration des médias » publié en 1992, l'harmonisation de ces mesures et l'institution d'un Conseil européen des médias chargé de veiller à l'application de ces règles ainsi harmonisées. A la suite de quoi, la Commission avait publié en 1996 un projet interne de directive pour la protection du pluralisme, qui s'attira les plus vives protestations de la part des Etats membres, en particulier pour violation du principe des compétences d'attribution, et fut relégué aux oubliettes. Une nouvelle offensive du commissaire Mario Monti en 1997, ainsi que la résolution afférente du Parlement Européen en 2002, ont subi le même sort - à juste titre.

La garantie du pluralisme doit être, en toute logique, centrée sur la "pertinence de l'impact sur l'opinion » (Meinungsrelevanz). Outre l'adoption de dispositions régulant la structure du marché, elle exige en effet des critères spécifiques aux contenus, ainsi qu'il ressort par exemple de l'art. 25 du Contrat d'Etat sur la radiodiffusion (Rundfunkstaatsvertrag). En référence à la jurisprudence du Tribunal constitutionnel fédéral, cet article stipule : «Aucun programme ne doit avoir un effet disproportionné sur l'opinion 
publique ». Or l'opinion publique ne peut être influencée en toute logique qu'à l'intérieur d'un espace de communication.

Cet espace existe-t-il au sein de l'UE ? Et existe-t-il une opinion publique européenne ? A la différence des Etats-Unis d'Amérique, l'Union européenne, de par le multilinguisme qui la caractérise, est et reste un ensemble hétérogène. Les grands médias qui ont un impact sur l'opinion sont conçus pour leur espace culturel et linguistique. Leur influence s'arrête aux frontières nationales; le journal télévisé de Sat1, les magazines Explosiv de RTL ou Plus Minus de l'ARD, n' ont pas la moindre influence sur la formation de 1'opinion en France.

En outre, chaque marché national de l'opinion est fortement déterminé par la structure de son système audiovisuel public et un certain nombre d'autres facteurs, dont l'organisation de la radio locale par exemple. De ce fait, je pense que c'est à l'échelon national que doivent se prendre les mesures de garantie du pluralisme ; elles y sont et seront plus efficientes que si elles étaient prises à Bruxelles par un Conseil des médias qui ne comprend peut-être même pas notre langue et qui connaît encore moins l'histoire de l'organisation de notre système médiatique.

Quant aux stratégies des Malone ou autres Murdoch, elles échappent par définition à toute approche européenne, et si on veut réguler l'action de ces groupes mondialisés, c'est en toute logique une solution internationale qui s'impose. Dans l'état actuel de l'intégration européenne, je ne vois aucun signe préfigurant un espace public européen, et donc aucune opinion publique qu'il conviendrait de protéger contre une opinion prédominante véhiculée par des groupes dont les activités s'effectuent au sein des différents Etats membres.

J'en conclus que nous devrions préserver au sein de l'UE cette hétérogénéité des mesures nationales visant à garantir le pluralisme. Le droit européen de la concurrence et les libertés fondamentales suffisent à assurer la libre concurrence nécessaire pour que se développe le marché européen des médias. Car ces dispositions ne s'appliquent pas seulement à la concentration horizontale, mais aussi à l'intégration verticale.

Néanmoins, nous devons accorder une attention particulière aux évolutions suivantes : quel impact aura la diffusion paneuropéenne de chaînes comme Eurosport, Euronews ou MTV d'une part, et d'autre part, quel sera celui de chaînes que leur format ou leur programmation destine à la réception simultanée dans plusieurs pays ? Il faut ajouter à ces chaînes la multiplication de formats d'émissions vendus et adaptés dans de nombreux Etats membres, comme l'émission de télé-réalité Big Brother (Loft Story en France) ou le jeu Qui veut gagner des millions ? Jusqu'à quel degré ira l'intégration verticale, notamment dans le segment de la distribution de droits ou d'œuvres cinématographiques?

Enfin, quel sera l'effet de la convergence numérique sur le pluralisme d'opinions ? Plus fondamentalement, l'avènement de l'ère du numérique soulève la question de l'efficacité des mesures établies en matière de garantie du pluralisme - et ce, quel que soit le niveau, communautaire ou national, où elles sont mises en œuvre. Autant d'éléments pour alimenter notre réflexion... 


\section{Discussion}

Joseph Lorent : Une remarque préliminaire : il conviendrait d'intégrer la presse écrite dans notre thématique. Nous entrons en effet dans une nouvelle ère de médias virtuels : une génération déjà a grandi avec Internet, les groupes de presse se sont transformés en opérateurs audiovisuels et s'étendent sur ce segment. A l'inverse, les chaînes de télévision et les stations de radio se font éditeurs ou deviennent partiellement propriétaires de médias de l'écrit. On observe en quelque sorte un 'mélimélange' quasi total. Les liens pluri-médias, le positionnement multimédias s'intensifient. Notre discussion ne peut donc pas non plus se limiter aux opérateurs publics ; elle doit également inclure les opérateurs privés. En d'autres termes : une approche intégrée du contrôle des concentrations est-elle possible et a-t-elle seulement un sens?

Werner Schwaderlapp : Avant toute chose, j'aimerais revenir à la question de départ : "Faut-il un droit des concentrations spécifique pour les médias à l'échelon européen ? » Ma réponse est : non, en aucun cas. La réponse à la question que vous venez de soulever, à savoir la prise en considération de l'ensemble du champ du numérique est donc également un «non ». Je reviendrai plus tard, si vous le permettez, sur la question européenne.

Peter Eberl : T. Kleist a très clairement présenté l'articulation entre, d'un côté, la prise en considération du marché par le droit communautaire de la concurrence et, de l'autre, celle du pluralisme d'opinions, qui relève des compétences nationales et de l'instrument qu'est le contrôle sectoriel, ce contrôle de concentration des médias tel qu'on l'entend en droit français ou allemand. Or la "pluralité des médias » est expressément considérée, par l'art. 21 du Règlement «Concentrations » (Règlement (CE) $n^{\circ} 139 / 2004$ du Conseil), comme un intérêt légitime permettant aux Etats membres de prendre des mesures appropriées dans ce domaine qui relève de la compétence exclusive de la Commission. Mais le catalogue des mesures qui relèvent des compétences des Etats membres est restreint et détaillé avec précision.

Il n'y a donc que très peu de domaines où les législateurs nationaux peuvent intervenir ; outre la garantie du pluralisme, il s'agit essentiellement de préserver des activités stratégiques comme les règles prudentielles ou de préserver la sécurité publique comme dans le cas de la défense et, maintenant dans une certaine mesure, la sécurité de l'approvisionnement de l'énergie. Non seulement ces domaines d'action sont limités, mais toute mesure nationale doit également être justifiée. Les Etats membres doivent au préalable informer la Commission, et celle-ci peut s'opposer aux mesures envisagées si elle estime qu'il s'agit d'un prétexte ou qu'elles ne sont pas proportionnées ou qu'elles sont incompatibles avec les principes généraux et les autres dispositions du droit communautaire.

\section{La Commission n'a aucune raison de se préoccuper d'une régulation spécifique}

En ce qui concerne un contrôle des médias à l'échelon communautaire, établi en parallèle aux autorités nationales ou en complément du contrôle de la concurrence effectué 
par la Commission, je partage l'avis de Werner Schwaderlapp. Les autorités nationales sont en effet mieux placées pour analyser la diversité de l'opinion. Elles en ont la compétence et connaissent beaucoup mieux ce domaine. Par ailleurs, la délimitation du marché communautaire par la Commission fait que les médias ne sont généralement pas pris en considération sous l'angle européen, tant les marchés des Etats-membres restent en quelque sorte régionaux ou nationaux. En l'absence d'un marché médiatique paneuropéen, la Commission n'a aucune raison de se préoccuper de la question.

J. Lorent : M Christiansen, vous venez d'un groupe positionné à l'échelon national et européen...

Olaf Christiansen : ... non seulement cela, mais aussi d'un groupe multimédias. La discussion porte sur l'européanisation des entreprises de médias. Je ne sais pas exactement ce que l'européanisation signifie dans ce contexte. Dans le domaine de la télévision ou de la presse, la détention de participations dans des entreprises dans plusieurs Etats membres suffit-elle pour parler d'européanisation? Je ne vois aucun segment du marché des médias qui existe au-delà des frontières nationales. L'offre est toujours déterminée par les zones linguistiques, les ensembles culturels... La diversité au sein de l'UE est telle qu'aucun marché médiatique communautaire n'existe. Par conséquent, il m'est difficile d'imaginer comment la Commission pourrait y avoir une quelconque compétence pour un contrôle des concentrations des médias.

Mais il ne suffit pas de se demander si un tel contrôle européen pourrait être nécessaire, il faut également se demander ce qu'il signifierait au plan matériel, c'est-àdire dans la pratique.

Par ailleurs, l'intégration d'autres médias, comme la presse et l'Internet, me paraît difficile. Cette semaine, dans le cadre d'un débat organisé par la Fondation FriedrichEbert sur le contrôle des opinions prédominantes à l'ère de la convergence (6 novembre 2006), a été débattue la question d'une régulation sous l'angle d'un "modèle médiatique global ». Et je me suis demandé par quel biais il pourrait être possible d'étendre à 'l'ensemble des médias' des compétences de régulation nées dans le seul domaine audiovisuel. Un contrôle selon le droit commun de la concurrence s'ajoutant au contrôle des concentrations spécifique à l'audiovisuel n'est-il justifié que lorsque la télévision est en jeu ? Je me demande si certains ne cherchent pas tout simplement à étendre sans le dire à d'autres médias les compétences qu'ils ont dans le seul domaine de l'audiovisuel.

J. Lorent : Je trouve le thème de ce Dialogue : "L'Européanisation des entreprises groupes médiatiques » un peu trop étroit, car ce monde virtuel des médias dans lequel nous entrons à pas de géants est déjà largement mondialisé ou globalisé. D'où ma question: ne menons-nous pas un débat d'arrière-garde si nous nous en tenons à la seule européanisation? Cela me rappelle l'ancien directeur de mon journal (Luxemburger Wort), qui aimait à rappeler les risques qu'il y a quand l'arbre cache la forêt. Et il citait toujours en exemple le siège de Constantinople, où les savants religieux discouraient sur le sexe des anges, pendant que les Turcs étaient en train de conquérir la ville. Ne sommesnous pas en train, nous aussi, de livrer ici un tel «combat d'arrière-garde »?

W. Schwaderlapp : La réponse est oui, bien sûr. Mais pourquoi le faisons-nous ? Parce que nous nous laissons guider par le souci de conserver les structures et non pas celui de préserver l'objectif de pluralisme. Notre conservatisme nous mène à nous interroger en maniant les seuls termes et outils que nous connaissons. Nous nous interro- 
geons sur la notion d'audiovisuel, sur un contrôle sectoriel de la concentration des médias à l'échelle européenne, voire au-delà de l'Europe. Mais nous ne nous demandons jamais réellement à quoi ressemblera le paysage médiatique vers lequel nous nous acheminons.

Pour en revenir au cas Berlusconi, je ne pense pas que le problème réside là où nous l'avons cherché. Il ne s'agit pas de se demander si on éprouve de la sympathie pour MM. Berlusconi, Murdoch, Saban ou d'autres. Mais si MediaSet rachetait ProSieben Sat.1, il résulterait de ce rapprochement un volume de chiffre d'affaires de l'ordre de 5 à 6 milliards d'euros. Ce volume n'a rien extraordinaire, il correspond au CA du Groupe RTL. Mais il ne résulterait pas de cette fusion un plus grand pouvoir médiatique dans quelque Etat membre que ce soit. Alors qui aurait à redouter une concentration des pouvoirs aux mains d'un tel groupe ? En premier lieu M. Blatter de la FIFA, ensuite Hollywood, car ce nouveau conglomérat négocierait à la fois des droits allemands et italiens, de la même manière que le groupe RTL doit négocier actuellement à la fois des droits français, anglais et allemands. Je ne vois pas où il pourrait y avoir un problème tant que l'Office fédéral des cartels et les autorités communautaires de contrôle des concentrations assurent un travail efficace.

Je ne vois pas non plus la nécessité de recourir à un contrôle sectoriel. Car un contrôle sectoriel signifie, et cela vaut surtout en matière de télévision, que nous avons à considérer un facteur de rareté temporelle. Bien que cela semble abstrait, cela est au contraire très concret. Comme la journée n'a que 24 heures, ce facteur de rareté crée pour le téléspectateur une situation différente de celle qu'il vit avec tous les autres types de biens. Ce temps limité, nous l'organisons, et nous le régulons en tenant compte des structures de pouvoir existantes. En ce qui concerne la consommation de la télévision, la structure de pouvoir à considérer est la chaîne ou le canal : nous nous sommes habitués depuis longtemps dans ce cas à l'idée que le vecteur (plates-formes Astra, Kabel Deutschland, etc.) et que les contenus ne font qu'un. Or il nous suffit de prendre en considération ce canal ou chaîne, d'y ajouter les quelques autres, et nous obtenons alors l'univers pertinent nous permettant de raisonner en termes de politique culturelle, et donc d'objectifs et de valeurs : diversité, culture et socialisation ou lien social.

Les plateformes, organisateurs de contenus, devraient-elles avoir un statut d'exception comparable à celui de l'audiovisuel public ?

Or demain, nous allons voir se conjuguer, du point de vue de l'utilisateur final, la communication en réseau, l'interpénétration entre communication individuelle et de masse. Les plateformes que nous aurons ne seront plus la simple addition de supports techniques, mais des organisateurs de contenus pour les utilisateurs. On peut résumer cette situation à venir par une formule : "plateforme = réseau de vecteurs x contenu ». L'émergence d'une telle plate-forme soulève de nouvelles questions en matière de rareté et de pouvoirs. Qui structurera ou organisera alors cette nouvelle situation ?

C'est-à-dire qu'il conviendrait de considérer ces plateformes de la même manière que l'audiovisuel public allemand qui dispose de certaines prérogatives en droit communautaire (et que l'Allemagne est loin de savoir épuiser...), prérogatives et un statut d'exception au droit commun de la concurrence que lui confèrent ses missions dans l'intérêt de la diversité, de la culture et de la socialisation des téléspectateurs. Autrement 
dit, il conviendrait de reconnaître que ces plateformes sont une catégorie médiatique pertinente qui doit bénéficier, de par les missions qu'elle remplit, d'un statut d'exception dans le champ d'application du droit communautaire de la concurrence.

P. Eberl : Qu'entendez-vous par statut d'exception pour ces plateformes?

W. Schwaderlapp : Je veux dire par là que l'organisation de ces plateformes (qui en est le responsable, comment doit-elle être structurée ?, etc.) doit pouvoir s'effectuer elle aussi dans une large mesure à l'échelon national. Je ne pense pas qu'il soit pertinent de réguler au niveau communautaire un type d'offre national, ce qui reviendrait à chercher à concilier artificiellement les habitudes de vie des Portugais, des Polonais ou des Français...

P. Eberl : Vous évoquez les plateformes au sens large, c'est-à-dire généralement basées sur Internet. Or il y a eu quelques cas où le contrôle des fusions européen a été appliqué à des plateformes de télévision payante. Ne peut-on en conclure que ce contrôle des fusions, qui s'est concentré sur le pouvoir des entreprises sur le marché, a eu pour corollaire (ou plutôt effet secondaire) de préserver le pluralisme d'opinions ?

La question s'est posée au milieu des années 1990, lorsque Bertelsmann, Kirch et la Deutsche Telekom avaient fondé en commun une plateforme ou un bouquet qui aurait été en situation de quasi-monopole dans la télévision payante en Allemagne, puisqu'elle était établie sur des technologies de cryptage propriétaires. Ce projet avait été interdit par la Commission. Un projet similaire, avec les mêmes acteurs, a ensuite vu le jour en Allemagne en 1998. Il a été à nouveau refusé, la Commission estimant que l'accès des tiers à cette plateforme était trop restreint. Voici pour l'Allemagne, mais la Commission s'est également opposée à un projet scandinave, a délivré une autorisation sous conditions en Italie, où Newscorp, l'entreprise de Murdoch, et Telepiù ont accepté de garantir le libre accès à leur plate-forme.

Le contrôle semble pourtant avoir fonctionné jusqu'à présent. Une situation de quasi-monopole pouvait être acceptée à condition que soit respecté l'accès des tiers, et que le marché reste ouvert. Ce critère est assurément fondamental en droit de la concurrence, surtout sur un marché hautement concentré comme c'est le cas en Allemagne et qui ne présente de perspectives que pour probablement tout au plus deux opérateurs de bouquets payants. Dans un tel contexte, il est primordial que la même technologie de cryptage et les mêmes décodeurs ou set-top-boxes soient accessibles à tous les acteurs, notamment pour éviter que le consommateur soit captif, mais aussi qu'un des acteurs dispose d'une prime au premier entrant. Les refus, ou plutôt les conditions exigées par la Commission ont eu pour effet d'éviter un certain cloisonnement du marché de la télévision payante.

W. Schwaderlapp : J'aimerais préciser un point. Je ne pense pas que la régulation doive s'effectuer à l'échelle nationale. Et par plateforme, j'entends ces nouveaux ensembles qui vont naître de la transition entre les médias de masse et la communication par les réseaux. Dans la description que j'en ai donnée, et qui prolonge les analyses faites par les participants de ce Dialogue, je me suis placé délibérément dans la perspective du consommateur final, car ces plateformes peuvent donner lieu à des abus de position dominante et, en tout cas, élever des barrières d'entrée sur le marché. Les médias de masse intègrent donc le facteur réseau dans leurs gènes par l'intermédiaire des évolutions actuelles - c'est cela que j'appelle plateforme. 
Et il est tout aussi pertinent d'appréhender ces nouveaux modèles que de se demander si l'Allemagne a besoin d'un audiovisuel public. Voilà pourquoi je souhaiterais que l'UE reconnaisse la plateforme comme un agrégateur de contenus à part entière. Reste à savoir comment organiser cette nouvelle offre, quelles règles définir. La question est complexe. Quoi qu'il en soit, il faut que l'Europe contrôle ce nouveau 'modèle', et qu'elle le contrôle mieux qu'elle ne le fait actuellement dans le cas du développement de l'audiovisuel public allemand vers une configuration d'agrégateur de contenus numériques, ou de plateforme.

O. Christiansen : Nous considérons naturellement que l'importance croissante des opérateurs d'infrastructures qui gèrent les plateformes est un aspect essentiel que nous devons intégrer dans notre approche du droit des concentrations. Et je pense que les instruments dont nous disposons au sein des Etats membres de l'UE ne prennent pas assez en considération ces évolutions. Mais je ne crois pas qu'il faille adopter une clause particulière pour le pluralisme des médias sur le modèle de l'art. 21 § 4 du Règlement sur le contrôle des concentrations. Je suis d'accord avec Peter Eberl sur le fait que le droit des cartels, qu'il soit communautaire, allemand ou français, s'applique également aux offreurs de plateformes et qu'il est suffisant pour garantir le libre jeu de la concurrence même s'il faut du temps pour qu'un acteur parvienne à faire valoir ses droits d'accès au marché.

Concernant la globalisation, je ne pense pas que les questions que nous abordons aujourd'hui soient exclusivement relatives au marché. Par ailleurs, nous sommes toujours contraints de définir le précisément le marché que nous considérons et de le délimiter sous l'angle du droit des concentrations. On aboutit alors toujours au marché national, de sorte que la question de la globalisation ne se pose pas de ce point de vue. Certaines entreprises sont présentes dans plusieurs pays, voire sur plusieurs continents, sans que les affaires qui y sont traitées de front ne soient obligatoirement conduites en un seul et unique lieu ou reliées entres elles. La philosophie de notre groupe a toujours été de considérer le monde dans sa diversité et d'apporter à chaque cas particulier une réponse spécifique, autonome. Nos différentes divisions coopèrent même trop peu entre elles, se trouvent plutôt même en situation de concurrence, ce qui ne me facilite pas la tâche au niveau de la direction centrale.

Et quand je considère la globalisation des autres groupes, comme ceux de Berlusconi ou de Murdoch, je ne peux m'empêcher de prendre fait et cause pour le droit allemand. Le droit allemand a en effet ceci de positif qu'il ne s'attarde pas sur l'origine des entreprises qui souhaitent se lancer dans la radio ou la télévision en Allemagne. J'aimerais qu'il en soit de même dans le monde entier.

\section{L'Europe reste faite de frontières : ce sont les langues et les mentalités}

J. Lorent : Pour en revenir au droit européen sur les concentrations... On a entendu dans l'introduction de ce panel que les limites nationales prévalent toujours dans le domaine de l'audiovisuel. Je ne partage pas ce point de vue. Je viens d'un pays où est établi un opérateur audiovisuel privé historique : RTL, qui s'est très tôt développé hors des frontières luxembourgeoises, d'abord à Paris avec la radio RTL, puis en lançant à partir de Luxembourg une station destinée aux îles britanniques (Radio Luxembourg), puis une autre destinée à l'Allemagne (RTL Radio Fröhliche Wellen), en instituant RTL 
Television en Allemagne, puis d'autres chaînes dans d'autres pays européens. Nous avons ainsi affaire aujourd'hui au plus grand groupe audiovisuel européen. Ce groupe ne connaît plus, ne connaît pas de frontières nationales. Il est soumis à la seule réglementation européenne, qu'il s'agisse de la Directive TSF ou du droit de la concurrence. Ce qui m'amène à ma question : les obstacles à une coopération transfrontières résideraient-ils dans le droit des concentrations ?

W. Schwaderlapp : Le cas de Radio Luxembourg est intéressant. Un groupe réalise, dans son pays d'origine, de nombreux programmes 'nationaux', c'est-à-dire aux contenus destinés à différents marchés. On pourrait envisager de même qu'une radio française diffuse un programme spécifique au marché allemand, ou l'inverse, qu'une radio allemande crée une station pour la France, etc. En théorie, tout est possible. Reste à savoir seulement s'il est judicieux du point de vue de l'entreprise allemande, par exemple, de servir le marché français avec des programmes radios produits à Berlin. Au Luxembourg, pays au cœur de l'Europe, vous parlez le Français, l'Allemand, et vous connaissez les diverses cultures et mentalités. C'est parce que le Luxembourg présente des circonstances particulières que vous avez pu déployer votre offre hors des frontières. Mais cet exemple peut difficilement être généralisé.

L'Europe est faite de frontières : celles des langues et celles des mentalités. J'ai eu jadis à piloter un groupement européen de coproduction d'œuvres audiovisuelles destinées à être diffusées dans les sept pays membres de ce groupement. France 2, qui s'appelait alors Antenne 2, en faisait partie. Nous avons tenté de définir des contenus pouvant convenir aux sept pays à la fois, en vain. Le seul sujet 'européen' en termes de contenu et pas seulement de financement que nous avons pu trouver fut une satire de l'Union Européenne intitulée The Gravy Train, et qui a été finalement produite par les... Britanniques.

Cette expérience est révélatrice. Les marchés médiatiques européens sont tellement différents les uns des autres (pas seulement du point de vue linguistique, mais avant tout du point de vue des cultures) que seul un haut niveau d'abstraction permet de développer des dénominateurs communs. Je m'en tiendrais ici avec les Américains, qu'on peut ne pas aimer pour certaines choses mais dont on ne peut qu' apprécier le pragmatisme, et qui ont cette belle formule : "If it ain't broke, don't fix it », s'il n'y a rien de cassé, il n'y a rien à réparer.

P. Eberl : La situation n'est guère différente du point de vue de la politique de la concurrence. Il faut toujours opérer une distinction entre les divers opérateurs sur le marché, qui parfois s'européanisent voire se globalisent comme RTL, MediaSet ou Newscorp (Murdoch). Il faut également prendre en compte le contrôle exercé par les autorités nationales sur les marchés pertinents.

Or ces marchés pertinents sont déterminés dans une large mesure par la demande. Dans le domaine des médias, celle-ci est liée en grande partie à la langue, mais aussi au contexte politique et culturel. Si TV5 présentait un débat sur un candidat socialiste à des élections en Allemagne, cela n'intéresserait presque personne dans les pays où est diffusée la chaîne. Dans le domaine du divertissement, c'est la même chose, certains formats peuvent fonctionner, mais ils ne connaîtront pas le même degré de succès selon les pays. C'est la raison pour laquelle l'offre de programmes diffère d'un Etat à l'autre et que les marchés médiatiques restent largement nationaux. Un groupe médiatique euro- 
péen ou international comme Bertelsmann peut, sans que cela pose normalement le moindre problème en droit, racheter une société de programmes dans un Etat où il n'est pas encore implanté. La concentration aussi reste une dimension nationale. En général, elle ne devient problématique que lorsqu'il s'agit d'acquisitions supplémentaires dans les pays où le groupe s'est déjà implanté.

O. Christiansen : Il n'y a effectivement pas de frontières nationales d'un point de vue juridique. Je suis tout à fait d'accord avec Peter Eberl sur le fait qu'il faille considérer la demande pour déterminer si le marché est national ou non, ou plutôt : la cible visée. Si l'opérateur luxembourgeois émet à partir du Luxembourg une offre destinée à l'Allemagne, son groupe-cible est évidemment le public allemand. Mais l'opérateur ne sert pas une demande plus grande que celle émanant des auditeurs allemands ; il n'accroît pas le groupe-cible. Mais il est vrai que son cas est particulier.

Dans l'introduction à ce Dialogue ont été évoqués le spill over et le principe du free flow of information. Il existe bien sûr des chaînes comme TV5 que l'on peut regarder en Allemagne et qui proposent des débats français, ou bien ARTE. Mais quand nous débattons de concentration des médias à l'échelle communautaire, nous parlons d'un tout autre public que des élites culturelles qui consomment ces chaînes et qui, parce qu'il s'agit d'un groupe restreint, n'entrent pas dans notre problématique.

Contre une régulation sectorielle européenne au-delà du droit de la concurrence ; mais pour une régulation « incitative » légitimée par la préservation du lien social

J. Lorent : Revenons-en à la question centrale : dans quelle mesure un droit sectoriel spécifique en matière de concentration des médias est-il nécessaire? Les intervenants ont déjà répondu en partie à la question mais je souhaiterais la poser sous un autre angle. Considérons-nous l'européanisation uniquement sous l'approche du marché ou dans une autre perspective encore - culturelle, comme l'a fait tout à l'heure Jacques Rigaud?

W. Schwaderlapp : Il faut à mon sens distinguer, lorsque nous évoquons l'organisation des paysages médiatiques, entre mesures répressives et mesures incitatives. Par mesures répressives, j'entends les conditions à l'obtention d'une licence, la limitation de certaines activités, etc. Par mesures incitatives, j'entends la promotion de la production cinématographique française ou allemande, de même que celle du service public.

Cette distinction me permettra de mieux répondre à votre question. En ce qui concerne les mesures répressives, j'ai déjà exposé mon point de vue : une régulation sectorielle européenne qui aille au-delà du droit actuel de la concurrence ne s'impose pas. En revanche, en matière de mesures incitatives, deux initiatives s'imposent à l'échelon européen. D'abord, le statut d'exception dont bénéficient les médias et l'audiovisuel par rapport au droit commun de la concurrence, légitimé par les objectifs de garantie du pluralisme, d'apport culturel et de lien social, doit être maintenu. Ensuite, l'Europe devrait, d'un point de vue économique, veiller à ce que la communication en réseaux s'organise autour d'entreprises européennes.

Je m'explique sur ce dernier point. Les plateformes (réseaux) organiseront l'accès à la communication à la fois individuelle et de masse. Les activités d'un Google nous donnent une idée partielle des enjeux. Les contenus qu'il met en ligne ne sont pas soumis au régime du droit d'auteur, puisqu'il se contente d'en rendre disponible l'accès. C'est là une réalité d'Internet, connue sous le nom de "réalité de contenu », et qui 
trouve une structure organisationnelle en la qualité d'un Google. Or la réalité de l'univers d'Internet revêt de si nombreuses formes qu'aucun de nous ne peut plus se passer d'un moteur de recherches Google ou MSN.

C'est ce schéma que je vois se transposer au monde des médias. Quand je veux trouver, dans l'offre pléthorique de la télévision, une émission susceptible de m'intéresser ce soir, il faut que je consacre une demi-heure à la lecture de mon programme TV, à passer en revue au fil des pages la programmation de toutes les chaînes. La situation se complique au fur et à mesure que l'offre se démultiplie (le réseau câblé du Land de Bade-Wurtemberg proposerait quelque 600 chaînes) et qu'elle se combine avec des services interactifs dépassant les traditionnelles offres téléphoniques (en Allemagne, le 0137) ou de consultation en ligne (9 Live), c'est-à-dire qui permettent au téléspectateur d'interagir avec le programme via son ordinateur, etc. Pour appréhender l'abondance de l'offre et les voies d'accès à celle-ci dans ce monde de communication en réseaux, l'analogie avec les Google s'impose.

Prenons un autre exemple, cette fois-ci dans l'univers analogique. Certaines personnes ne regardent plus du tout les chaînes publiques de l'ARD et de la ZDF, d'autres ne regardent pratiquement pas les chaînes commerciales comme Sat.1 et RTL. C'est là une manière pour le consommateur de réagir à l'abondance de l'offre sans avoir à passer une demi-heure à lire le programme TV. Il économise son temps et est assuré à $80 \%$ de trouver une offre qui lui convient. Le choix se fait ainsi par l'intermédiaire d'une marque.

Si nous revenons maintenant à l'aspect du pluralisme dans la dimension Internet, alors nous constatons que nous avons besoin d'une sorte de Google appliqué à la télévision. Considérer un tel système uniquement sous l'angle économique - le nombre de 'clics' qu'il génère - relève de l'absurdité. Mais intégrer à ce Google télévisuel un élément qui faciliterait la visibilité dans la richesse de l'offre, voilà en revanche un objectif de politique culturelle qui va dans le sens d'une organisation des médias fondée sur l'incitation et non sur la répression.

Voilà l'approche que je défends à la fois pour des raisons d'ordre culturel et des raisons de politique structurelle. Car les opérateurs européens du satellite, du câble, les offreurs de contenus mènent une stratégie de défense de leurs propres intérêts. Pourquoi participeraient-ils donc à une telle œuvre d'orientation commune ? Je récolte les bénéfices de ma position dominante sur le marché, alors que mes concurrents rencontrent des difficultés - voilà comment ils pensent. Et c'est pour cela qu'il faut prendre des mesures incitatives. Pour que la puissance virtuelle de ces Google ait un sens. Un YouTube, par exemple, Norbert Schneider l'a évoqué tout à l'heure, a atteint une valorisation boursière de 1,65 milliards de dollars, en l'espace de 18 mois seulement...

P. Eberl : Je voudrais juste ajouter une précision au sujet de Google et du fait qu'il ne donne que des liens vers des contenus sans avoir à payer de droits d'auteurs. Cette pratique a fait l'objet récemment d'une plainte en référé déposée par des éditeurs de presse belges contre Google, avec succès.

Pour ce qui est de ces filtres ou de ces aides à l'orientation dans la multiplicité des canaux, ces activités vont se développer. Un peu comme dans le segment du support papier se sont multipliés les magazines télé. Or ces éditeurs servent une demande nationale en Europe. De même, dans leur propre intérêt, les éditeurs de presse et les opérateurs audiovisuels européens vont se mettre à proposer des electronic program guides, 
de sorte que cela ne tombera pas forcément entre les mains d'un Google ou autre moteur de recherche.

J. Lorent : Bertelsmann pense-t-il uniquement au commerce ou aussi à la culture ?

O. Christiansen : Nous pensons évidemment aussi bien à l'aspect culturel qu'à l'aspect commercial ; ce sont les deux faces d'une même médaille pour un groupe médiatique. Point n'est besoin de régulation pour augmenter la diversité culturelle dans les programmes télévisés, celle-ci est suffisamment garantie.

Mais il faudra une telle régulation pour le numérique. Car l'interactivité, la possibilité de s'adresser à des groupes-cibles et de leur proposer une offre spécifique que permet la numérisation, donc la segmentation et l'individualisation de l'offre vont faire naître le besoin de structurer ces services afin que le consommateur puisse s'y orienter et trouver l'offre qui lui convient. Mais je ne sais pas quelle configuration il conviendra de donner à un tel système.

En en appelant à " Bruxelles », ne sommes-nous pas en train d'ouvrir une boîte de Pandore ?

J. Lorent : Ne serions-nous pas en train de jouer à l'apprenti sorcier en réclamant une réglementation européenne dans le domaine par nature sensible des médias ? Pour le dire autrement: ne sommes-nous pas en train d'ouvrir une boîte de Pandore qu'il nous sera ensuite impossible de refermer ? Faut-il réellement en appeler à « Bruxelles » alors même que nous ne savons pas encore comment va évoluer le paysage des médias?

W. Schwaderlapp : Je pense que ne doivent être régulés à l'échelon communautaire que quelques rares points parmi ceux que nous avons soulevés dans le cadre de cette problématique...

J. Lorent : ... l'Europe veut peut-être aller plus loin ?

P. Eberl : En tant que représentant de la boîte de Pandore potentielle, je pense que le contrôle du pluralisme doit s'effectuer à l'échelon national. Il y a certainement quelques personnes à la Commission qui voient les choses autrement. Mais si une demande est émise par la société civile et que cette demande ne peut trouver de réponse qu'au niveau européen, alors la Commission sera prête à établir des propositions qui devront ensuite être approuvées par le Parlement et le Conseil qui impliqueront les Etats membres dans la discussion.

O. Christiansen : Le débat sur la concentration dans les médias est en cours à l'échelle nationale, et Bruxelles de son côté traite du sujet dans les détails. Je ne sais pas si ces débats vont converger. En Allemagne, ou en France avec le Rapport de la Commission Lancelot, nous débattons de nombreux modèles différents. Je ne sais pas si c'est le bon moment pour lancer le débat à l'échelon européen. Je crains qu'il n'en sorte un système quelque peu chaotique qui risque de n'être pas opérationnel.

Dans notre propre intérêt, nous souhaitons qu'il existe un système suffisamment transparent pour que les entreprises puissent évaluer ce qui est faisable et ce qui ne l'est pas (c'est déjà assez difficile avec le droit des cartels allemand). Mais je pense qu'il est prématuré pour l'instant d'aller au-delà des débats en cours sur la révision de la Directive TSF.

J. Lorent : Dans le dossier préparatoire à ce colloque, Isabelle Bourgeois a fort justement évoqué la diversité culturelle. N'est-elle pas en effet l'un des atouts majeurs d'une vision de l'intégration européenne conformément à la devise : "l'unité dans la 
diversité »? Dans le domaine des médias, les enjeux vont bien au-delà de la diversité culturelle, et bien au-delà aussi du principe de subsidiarité. Ils concernent le pluralisme des opinions et un point dont nous n'avons guère parlé jusqu'à présent : la liberté de la presse en général.

Au risque de me répéter : les médias sont un champ très sensible. Et ce champ ne relève pas en priorité des législateurs nationaux, ni du droit européen, mais d'abord et avant tout de l'article 11 de la Charte des droits fondamentaux et de l'article 10 de la Convention européenne des droits de l'Homme. C'est pour cette raison que la Commission reste très prudente en la matière et préfère se tenir en retrait, voire à l'écart comme me l'a expliqué récemment Viviane Reding, la Commissaire en charge de la politique des médias et de la société de l'information, et qui fut pendant de nombreuses années ma collègue au sein de la rédaction du Luxemburger Wort.

Voici ma question : Le moment est-il opportun pour mettre en place un droit européen de la concentration des médias européen ? Et une telle action n'est-elle pas susceptible, directement ou indirectement, de porter atteinte au pluralisme des opinions et à la liberté de la presse?

W. Schwaderlapp : A mon avis, une réglementation européenne ne pourrait se justifier que dans deux cas. D'une part, si des groupes procèdent à des fusions et que le droit européen de la concurrence ne parvient pas à appréhender cette nouvelle concentration des pouvoirs - une situation que j'ai néanmoins du mal à imaginer. Donc si le droit commun européen tel qu'il existe échouait dans sa mission, alors il faudrait envisager un autre modèle. D'autre part, il faudrait également qu'il existe un espace public européen, un Figaro européen, une ZDF européenne, que les barrières linguistiques tombent, etc. C'est alors seulement, si ce cas se produit - ce dont je doute fort -, qu'il conviendrait de faire intervenir l'échelon communautaire.

P. Eberl : Une précision en ce qui concerne le contrôle des fusions dans la presse. Il y a à l'échelle européenne relativement peu de cas de fusions, car nous ne sommes compétents que si un certain seuil de chiffre d'affaires est atteint par la nouvelle entité. Or les groupes de presse atteignent rarement les seuils de chiffres d'affaires à partir desquels une fusion est soumise au contrôle de la Commission. En outre, comme la presse est normalement une activité nationale, la clause des deux tiers est souvent appliquée, c'est-à-dire que quand toutes les entreprises intéressées réalisent les deux tiers de leur CA dans un Etat membre, l'autorité nationale (l'Office fédéral des cartels pour l'Allemagne) est compétente. C'est pour cette raison que nous avons eu à connaître de très peu de cas de fusions dans la presse.

O. Christiansen : A la différence de la Commission, l'Office fédéral des Cartels a à connaître de très nombreux cas de fusions dans le domaine de la presse, de cas concernant l'audiovisuel sur les marchés locaux en partie, car il existe en Allemagne une clause spécifique à ces activités, liées généralement à des entreprises de taille moyenne ou petite, et selon laquelle le seuil de CA à partir duquel s'opère le contrôle est multiplié par vingt.

Mais pour en revenir à la question d'un contrôle européen de la concentration des médias, je pense que c'est une approche trop hâtive. En tant que groupe médiatique, nous nous soucions par définition de la liberté de la presse. L'idée même d'un élargissement de la régulation nous préoccupe beaucoup en ce sens. 
Il faut au contraire promouvoir la concentration, la naissance de groupes européens !

Jacques Rigaud (dans la salle) : Un mot seulement sur l'intitulé de cette table-ronde : "Les entreprises de médias s'européanisent. Faut-il un contrôle des concentrations?" Je trouve que le sujet est très mal posé, pardonnez-moi, car nous manquons d'entreprises européennes.

Les entreprises de médias ne sont pas suffisamment européennes, pas suffisamment puissantes, on l'a dit, par rapport à la mondialisation. Et par rapport au fait qu'un certain nombre d'entreprises américaines sont mondialisées, l'Europe est déjà provinciale en tant que telle. Je souhaite en tant que Français et en tant qu'Européen qu'il y ait de grands groupes de médias en Europe qui soient capables d'atteindre la dimension mondiale. La question que je me pose, c'est comment la renforcer. Et la question que je pose à l'UE, c'est comment aider à la concentration des médias ? Et ne nous lançons pas une fois que plus dans un processus que je croyais spécifique à la France, qui consiste à réglementer, à limiter, à interdire, à se poser le problème du contrôle des concentrations.

Le monde des médias, ça a été dit à plusieurs reprises, que ce soit dans le domaine de la radio ou de la télévision, est très tributaire des réalités nationales. Vous l'avez dit à l'instant, s'il y avait un Figaro européen, le problème se poserait en des termes différents.

Je vais vous donner un exemple : il y avait, à l'époque où je suis arrivé à la CLT, RTL Télévision-Télé Luxembourg qui émettait sur 3 régions francophones ; il n'y avait même pas l'obstacle de la langue. C'était le Grand Duché de Luxembourg, où le français est une des langues reconnues, ainsi que la Wallonie et la Lorraine. On s'est rendu compte que ce programme unique n'avait pas un contact suffisant avec son public parce que les Belges ne dînent pas à la même heure que les Français et parce qu'un journal où on explique la grève des dentistes belges n'intéresse pas les Lorrains. De la même manière, les Belges se moquent des problèmes de la Lorraine. Les Luxembourgeois, même s'ils s'intéressent à leur puissant voisin, aiment qu'on leur parle du Luxembourg. On a fini par faire de cette télévision trois télévisions. Pendant des années, comme on avait une équipe à Bruxelles, par tous les temps, vers 5 heures de l'après-midi au plus tard, on envoyait les sujets à Luxembourg, les sujets belges, pour être traités dans le journal de 19 heures. On a créé RTL TVI à Bruxelles, qui est devenue une chaîne belge, sur le câble belge et reconnue en Belgique. On a créé RTL Télévision pour le grand Duché de Luxembourg, on a créé un programme en français pour la Lorraine, ce qui montre bien qu'il faut coller, pas seulement à la langue, mais aux habitudes, aux références culturelles et sociales d'un public.

En revanche, sur un certain nombre de problèmes, il peut y avoir une vision stratégique, et je regrette qu' on n'ait pas suffisamment distingué, dans toutes nos discussions, le niveau managérial, celui de la stratégie économique et financière et celui des contenus. Dans le domaine des entreprises européennes des médias, la règle d'or doit être qu'il doit y avoir une profonde unité de références, de méthodes et d'évaluation des résultats en termes économiques et financiers, qu'il peut y avoir des services communs en ce qui concerne les achats de droits ou, pour certaines productions de haut niveau, un travail en commun.

Et puis le niveau des contenus, qui doit coller aux réalités nationales, voire régionales. Autant dans le domaine de l'industrie automobile ou pharmaceutique, une entreprise 
européenne va fabriquer le même produit, les Renault ou VW que l'on vend de part et d'autre du Rhin sont, que je sache, exactement les mêmes voitures ; il n'y a que les notices d'emploi qui changent. En revanche, dans le domaine de l'audiovisuel, il peut y avoir à la fois des produits généralement de haut niveau qui peuvent être communs avec les traductions, voire des concepts de jeux qu'on peut transposer d'un média à l'autre, mais une grande diversité culturelle et de contenu et une grande unité au niveau de la stratégie. Mais avant de se poser le problème du contrôle des concentrations, concentrons les entreprises et donnons leur le moyen d'être à la fois européennes et si possible mondiales.

\section{La diversité culturelle au sein de I'UE contribue au pluralisme}

Un intervenant (dans la salle) : Je ne suis malheureusement pas Suisse, mais je suppose qu'un Suisse répondrait autant que vous par la négative à cette question de la nécessité d'un contrôle européen de la concentration des médias. La Suisse reçoit les chaînes de ProSiebenSat.1 et peut-être aussi de MediaSet. Et maintenant, ces deux entreprises fusionnent. Vous n'avez pas répondu à la problématique des petits pays. Je demanderais bien aux représentants de l'UE comment ils ont pris en compte la problématique des petits pays.

P. Eberl : Il faudrait d'abord que le Suisse adhère à l'UE...

Mais je répondrai d'abord à Jacques Rigaud : l'UE favorise les concentrations transfrontalières. D'abord, comme Thomas Kleist l'a exposé, il y a les libertés fondamentales, la libre circulation des services et des capitaux, et s'il y a des mesures de protectionnisme de la part de quelques Etats membres, il existe des procédures contre ces Etats membres. Dans un tel scénario, la Commission peut porter plainte auprès de la Cour de justice au Luxembourg. De même, dans le contrôle des concentrations, il y a une clause qui confère à la Commission la compétence exclusive pour l'analyse de la fusion notifiée.

La Commission a les pouvoirs de défendre cette compétence si les Etats membres essaient de prendre des mesures protectionnistes, comme ça a été le cas dans la fusion E.ON-Endesa dans le secteur de l'énergie en Espagne, où le gouvernement espagnol a augmenté les compétences de l'autorité de régulation de l'énergie. Cette autorité a imposé des conditions, invoquant l'article 21 , au nom de la sécurité nationale. Elle a imposé ainsi une vingtaine de conditions, puis la Commission a adressé une lettre officielle au gouvernement espagnol et a menacé de traîner l'Espagne devant la Cour de Justice européenne, à la suite de quoi le gouvernement espagnol a abandonné ses conditions.

S'il y a un protectionnisme qui porte atteinte à la libre circulation et au marché unique, la Commission intervient contre les Etats membres. Il y a une intervention de la Commission lors de projets de fusions uniquement s'il y a un problème au niveau de la concurrence. Ces problèmes surgissent, vu la structure nationale des marchés, quand il y a des chevauchements au niveau national. Si Bertelsmann achète une chaîne de télévision ou de radio en Allemagne, il y aura probablement des problèmes. Mais s'il achète une chaîne en Suède, a priori, il n'y a pas de problème. Le droit de contrôle des concentrations et le Traité de l'UE est à mon avis très favorable à une intégration par-delà les frontières.

Mais pour créer des champions européens, l'initiative doit émaner des entreprises. Ce n'est pas la Commission qui peut créer des grands groupes de médias. La seule 
chose que l'on peut faire, ce sont des programmes de coopération dotés de fonds, comme le programme MEDIA.

Vous avez aussi évoqué les achats de droits. Là encore, il s'agit de marchés nationaux : le football italien intéresse au premier chef les Italiens. Il y a les droits de football nationaux, mais aussi les droits de la Champions' Ligue, qui sont vendus au niveau national. En ce qui concerne la Premier League, la Commission est intervenue pour que BSkyB (Murdoch) n'attire pas à lui la totalité des droits mais qu'il y ait des droits pour certains matches chaque semaine pour des tiers, donc pour qu'il y ait une certaine concurrence.

J'en viens maintenant à la problématique des petits pays. L'analyse est la même. La Suisse n'étant pas membre de l'UE, imaginons par exemple le cas d'une fusion francoallemande de deux chaînes qu'on peut capter au Luxembourg. Dans ce cas, la Commission considère les parts de marché que ces entreprises candidates à la fusion réalisent au Luxembourg, en France et en Allemagne, qu'il s'agisse des parts de marché publicitaire ou des parts d'audience. Ainsi, les petits pays ne sortent pas du cadre.

Supposons que la Suisse soit membre de l'UE. Il serait très regrettable pour ce pays que la pluralité dépende du fait que les Suisses se voient obligés de regarder des programmes italiens ou français dans le cas où il n'existerait pas de programmes proprement suisses. C'est plutôt un avantage pour les Suisses de pouvoir recevoir tous ces programmes en quelque sorte par hasard, grâce au spill over. Ils constituent un complément bienvenu à leurs propres règles garantissant la liberté et le pluralisme d'opinions.

W. Schwaderlapp : Etant donnée la taille réduite du marché, le nombre de programmes suisses est limité. Les Suisses ont de nombreux programmes étrangers car ils peuvent capter les programmes de cet espace trilingue et qu'ils les acceptent. Il s'agit d'une situation un peu particulière car il s'agit d'un pays où trois langues se côtoient. Le cas de l'Autriche, bien sûr, est similaire. Mais dans ces petits pays, il faut rappeler aussi que le système public se porte plutôt bien, à l'exclusion d'ATV, dont je ne sais pas si elle existe encore.

L'adaptation des formats et programmes est une réponse à la diversité des cultures

Isabelle Bourgeois (dans la salle) : Vous n'avez répondu qu'en partie à la question de Jacques Rigaud et êtes resté, pour ainsi dire, au niveau managérial. Or Jacques Rigaud parlait des différences culturelles, des structures mentales, des différentes habitudes de vie, des différentes gestions du temps...Comment une entreprise du secteur des médias gère-t-elle cette diversité ?

W. Schwaderlapp : La réponse du point de vue de l'entreprise, formulée de manière abrupte et ne prenant en compte que l'aspect purement économique, est qu'il lui faut créer des produits réutilisables sur d'autres marchés (d'autres pays) en partant d'un seul investissement et en réduisant au maximum les coûts d'adaptation des produits.

Nous avons déjà évoqué Qui veut gagner des millions? ou Big Brother. J'ai rappelé les difficultés que nous avons rencontrées dans le temps au sein du Groupement européen de co-production. Et le constat que nous y avons fait : l'œuvre nationale est toujours beaucoup mieux reçue que l'œuvre étrangère, quelle que soit l'attention apportée à la qualité du doublage de celle-ci.

Il existe peut-être quelques exceptions, mais en règle générale, c'est cette situation qui prévaut. Du reste, la langue prédominante au sein de la télévision à l'échelle 
mondiale est l'anglais américain, c'est un fait établi, tout comme le haut degré d'acceptation des Européens à l'égard des produits américains, facteurs de nivellement, si je puis me permettre de m'exprimer en faveur des Européens. En tout cas, ils préfèrent ces produits-là à ceux de leurs voisins européens.

En ce qui concerne l'adaptation des programmes, il y a de multiples approches, liées au 'format'. Généralement, j'ai un produit audiovisuel qui ne consiste pas en une émission, d'une durée de 90 minutes par exemple, mais en un projet de structure, une idée générale en vue de la production. Je peux donc réaliser ce projet de sorte qu'il devienne un produit adapté aux pays respectifs. Cela dépend de la grille des chaînes qui le diffuseront. Le packaging est ainsi un élément très important du point de vue de la rentabilité mais aussi du point de vue culturel.

Si par exemple j'obtiens pour Big Brother (l'adaptation française s'appelle Loft Story) un créneau d'une heure par jour sur une grande chaîne, cela déterminera les possibilités d'adaptation de ce format, de même que mes possibilités seront différentes si je dispose d'un créneau sur une petite chaîne. Maintenant, il existe aussi des formats comme Deutschland sucht den Superstar (diffusé en France par M6 sous le nom de Nouvelle Star) qui s'adaptent de manière quasi-automatique aux conditions nationales car elles invitent le public national à y participer. Il existe par ailleurs des émissions dont l'adaptation est strictement réglementée par la licence d'exploitation, ce qui est le cas du jeu Qui veut gagner des millions? L'inventeur britannique de ce format a précisé par exemple dans le détail (comme Andrew Lloyd Weber pour ses comédies musicales) qu'il devait y avoir 27 types d'éclairage et 33 mélodies différentes et que la mélodie $\mathrm{n}^{\circ} 27$ devait être jouée à un moment bien précis.

Pour conclure, comme Jacques Rigaud l'a expliqué pour Radio Luxembourg, les entreprises cherchent à réaliser des produits destinés à plusieurs marchés tout en faisant en sorte que cela leur coûte le moins possible, c'est-à-dire en produisant des programmes communs à plusieurs pays lorsque cela est possible (quel que soit le niveau considéré dans le processus de production et de commercialisation). Mais lorsque cela s'impose, nous adaptons le produit en conséquence.

J. Lorent : Nous pouvons conclure de notre discussion qu'un droit européen de la concentration spécifique aux médias n'est actuellement souhaité par personne, dans le sens où il a été débattu aujourd'hui. Nous devons donc manier avec prudence cette virtuelle boîte de Pandore. 


\section{Postface}

\section{Construire la démocratie de la communication}

Si j'essaie de résumer le contenu de ces deux derniers jours, un exercice assurément subjectif, je commencerai par un constat. Nous sommes tous d'accord pour dire que nous vivons actuellement une troisième révolution copernicienne du monde des médias. Après celui de Gutenberg, puis celui d'Elektron avec l'apparition des médias de l'écrit puis des médias électroniques à partir des années 1920 avec d'abord la radio et ensuite Internet. Les révolutions coperniciennes sont très utiles, surtout dans le domaine de l'astronomie. Elles ont permis de démontrer que la Bible n'est pas un manuel de sciences naturelles et nous ont transmis une image réelle du monde qui nous entoure. Nous avons constaté durant ce Dialogue franco-allemand sur les médias d'importants changements structurels dans les médias à proprement parler, et aussi dans le financement des médias.

Le deuxième point que je retiendrai a été développé de manière très claire par JeanLouis Missika et peut se résumer par cet adage que nous connaissons bien : s'il existe une certitude dans la vie des Hommes, c'est bien celle de l'incertitude. Nous ne pourrons jamais tenter de modeler l'avenir que pour en réduire les incertitudes. Si cela est inconfortable, cela présente aussi des avantages. Les évolutions que nous avons abordées sous les angles les plus divers révèlent toute l'ambivalence de la vie humaine. C'est à la lumière de cette ambivalence que nous devrons considérer l'évolution future des médias.

Le troisième point à retenir de ce Dialogue est que, malgré tous les points communs que partagent la France et l'Allemagne (pas seulement pour des raisons de proximité géographique, mais aussi pour des raisons culturelles), nos deux pays restent très différents. Les participants allemands auront beaucoup parlé d'approche du marché et de ce marché lui-même, y compris dans la perspective des sciences économiques théoriques (Dieter Schmidtchen). A l'opposé, Alain Lancelot a rappelé que le rôle du marché est considéré en France comme un «trou noir », si vous me permettez de filer la métaphore astronomique. Or un trou noir permet la création de nouveaux mondes. Tandis que plusieurs intervenants se sont prononcés en faveur de la dérégulation des compétences en matière de médias, Jacques Rigaud a évoqué la possibilité d'autoriser un plus fort degré de concentration dans les médias afin de favoriser la création de grands groupes au niveau européen. Les moyens que nous avons à notre disposition pour empêcher la concentration dans les médias sont limités.

Un quatrième point a surgi au fil des débats, et il me semble primordial : celui de l'éducation. J'ai pu constater en Allemagne, comme l'a fait Jean-Louis Missika pour la France, que la classe politique a grand besoin d'apprendre ce que sont les médias et quelles lois régissent leurs activités. Et les représentants de la 'classe universitaire' présents à nos débats pourraient utilement rappeler aux responsables politiques français et 
allemands que si les médias diffusent leur message, que si le politique est en situation d'interdépendance avec le monde médiatique, la 'classe politique' manque cruellement de ce savoir indispensable pour comprendre réellement les effets des médias et leur évolution. En Allemagne, nous avons compris depuis longtemps que, pour contrer les effets de la concentration des médias, il faut développer les compétences médiatiques des citoyens et consommateurs, donc leur dispenser les savoirs nécessaires à la compréhension des lois inhérentes à la communication et au monde des médias. Ce sont là les nouvelles compétences requises non seulement pour que chacun puisse s'orienter dans l'immensité de l'offre, choisir un contenu en connaissance de cause, mais bien plus généralement aussi celles qu'il nous faut à tous pour entrer de plain pied dans l'ère de l'information.

Victor Henle 


\section{LES INTERVENANTS}

\section{Francis Balle}

Professeur à l'Institut Français de Presse (IFP, Université de Paris 2); directeur de I'Institut de recherches et d'études sur la communication (IREC)

Diplômé de l'Institut d'études politiques de Paris. 1963-67 : professeur de philosophie au lycée français d'Oran, puis assistant à l'Université d'Alger. 1967-1972 : assistant de Raymond Aron, puis maître-assistant à la Sorbonne. 1976-1986 : directeur de l'Institut Français de Presse. 1986-89: Vice-chancelier des Universités de Paris. 1989-93: membre du Conseil supérieur de l'audiovisuel (CSA), en charge du spectre hertzien, des nouvelles normes et des nouvelles technologies. Depuis 1981 : professeur invité à l'Université de Stanford (Californie). Depuis août 2004 : membre du Conseil d'administration de Radio France. Nombreuses publications, notamment «Médias et sociétés » (1980, régulièrement actualisé).

\section{Isabelle Bourgeois}

Chargée de recherches au Centre d'Information et de Recherche sur l'Allemagne Contemporaine (CIRAC) ; maître de conférences à l'Université de Cergy Pontoise.

Née en 1955. Ancienne élève de l'Ecole Normale Supérieure (Fontenay-aux Roses). 1980-1985: lectrice à l'Université de Hanovre. 1985-1988: attaché culturel près l'Ambassade de France à Bonn, chargée de l'audiovisuel, puis chargée de mission à la Direction générale des relations culturelles, scientifiques et techniques du Ministère des Affaires étrangères (Coordination Europe, puis Direction de la Communication). Depuis novembre 1988 : chargée de recherches au Centre d'Information et de Recherche sur l'Allemagne contemporaine (CIRAC). 1989-2001: chargée de cours à l'Institut d'études politiques de Paris. Depuis 2002 : maître de conférences à l'Université de Cergy Pontoise. Domaines d'activité : questions économiques et sociales, médias/communication, économie du savoir, innovation, études comparées France/Allemagne dans ces domaines. Nombreuses publications en France et en Allemagne. 1990-2006 : correspondante du bulletin spécialisé «epd-medien». Depuis 2000 : rédactrice en chef de Regards sur l'économie allemande (Bulletin économique du CIRAC).

\section{Olaf Christiansen}

Avocat spécialisé dans le droit des concentrations, Bertelsmann AG

Né en 1971. Etudes de droit à Würzburg et à la Penn State Dickinson School of Law, USA. Stage de fin d'études à Hambourg. Avocat dans un cabinet international à Berlin, 
avant de rejoindre la Bertelsmann AG en 2002. Au sein de la direction Droit des sociétés du Groupe Bertelsmann à Gütersloh et Bruxelles, en charge du droit des concentrations et de ses domaines connexes, comme le droit des médias et de leur concentration. Suit principalement les procédures de contrôle des fusions au niveau allemand et européen, ainsi que les questions juridiques internes liées aux concentrations dans les domaines télévision et radio, presse quotidienne et périodique, magazines, livres et musique, ainsi que dans les activités d'impression et de services.

\section{Prof. Dieter Dörr}

Président de la Kommission zur Ermittlung der Konzentration im Medienbereich (KEK: Commission d'évaluation de la concentration dans le secteur des médias ; Potsdam)

Né en 1952 à Tübingen. Etudes de droit, Universités de Sarrebruck et de Cologne, puis Professeur à 1'Institut des affaires internationales de 1'Université de Hambourg. 199095 : Directeur juridique du Saarländischer Rundfunk (établissement de radiodiffusion de droit public de la Sarre). Depuis 1995, titulaire de la Chaire de Droit public, Droit international et européen et de Droit des médias à l'Université Johannes-Gutenberg de Mayence. Depuis 2000, Directeur de l'Institut des Médias de Mayence. Depuis 2000, membre de la KEK, puis président depuis 2004. Parallèlement (fonction secondaire), depuis 2003, Juge auprès du Tribunal supérieur régional de Coblence.

\section{Peter Eberl}

DG Concurrence, Commission européenne (Bruxelles)

Etudes de droit et d'économie à l'Université de Bayreuth et en France (maîtrise de droit international, Aix-en-Provence, et D.E.A de droit communautaire, Rennes). Avant de joindre la Commission européenne, Peter Eberl a travaillé pour des cabinets internationaux d'avocats à Bruxelles et à New York, pour l'ART (aujourd'hui l'ARCEP) en France et dans le secteur public en Allemagne. Peter Eberl est fonctionnaire européen au sein de la Direction Générale de la concurrence à Bruxelles. Il est actuellement membre de l'unité en charge des questions horizontales et de cohérence dans le domaine du contrôle des fusions et de l'anti-trust. Récemment, il a travaillé sur des fusions concernant les systèmes de navigation et des cartes numériques ainsi que des concentrations dans les marchés de la publicité online. En outre, il a suivi des cas anti-trust concernant les droits d'auteur et la musique en ligne.

\section{Elisabeth Flüry-Hérard \\ Membre du Conseil Supérieur de l'Audiovisuel (CSA, Paris)}

Ancienne élève de l'Ecole normale supérieure et de l'ENA. 1982-86 : Administrateur civil des services du Premier ministre. 1986-88 : Mise à disposition du Secrétariat général du comité interministériel pour les questions de coopération économique européenne (SGCI). 1989-93 : Déléguée aux affaires européennes, puis directeur des affaires internationales au Centre national de la cinématographie (CNC). 1993-94 : Conseiller tech- 
nique au cabinet de Jacques Toubon, ministre de la Culture. 1994-98 : Directeur de la production cinématographique, puis directeur du cinéma au CNC. 1998-2002 : Directeur général de l'Institut français du cinéma et des industries culturelles (IFCIC). 19992002 : membre du Conseil de la concurrence. Depuis avril 2002 : membre du CSA.

\section{Prof. Jürgen Heinrich}

(Université de Dortmund)

Etudes de sciences économiques et de gestion à Kiel et Tübingen ; soutenance de thèse à Kiel. Collaborateur de l'institut de recherche économique Institut für Weltwirtschaft (IfW, Kiel) et maître de conférences à l'université de Kiel. Depuis 1980 : Chaire de journalisme économique à l'Université de Dortmund. Domaines de spécialité : économie des médias, macro-économie et journalisme économique.

\section{Victor Henle}

Président de la Thüringer Landesmedienanstalt (TLM : autorité de régulation du secteur privé des médias du Land de Thuringe)

Né en 1942. Etudes de droit à Munich et Paris. Diverses fonctions dans l'administration du gouvernement de l'Etat libre de Bavière. 1985-89: journaliste dans un quotidien bavarois, développement des activités de l'éditeur et direction (filiale radio, radio locale et câblo-opérateur régional). 1989-90: au sein du ministère de l'Intérieur de Bavière, création et direction d'un mensuel pour la police bavaroise. 1990-92: participation à l'institution du ministère de l'Intérieur de Thuringe, puis porte-parole. 1992-2007, Président de la TLM. Depuis 1999, vice-président de la Direktorenkonferenz der Landesmedienanstalten (DLM : conférence permanente des directeurs des autorités de régulation des médias des Länder) et président de la Konferenz der Direktoren der Landesmedienanstalten (KDLM : conférence permanente des directeurs en charge de la garantie du pluralisme). 1993-2007, membre de la Commission Protection de la jeunesse et de la Cellule commune des Landesmedienanstalten pour les questions relatives aux programmes, à la publicité et à la compétence médiatique.

\section{Prof. Bernd Holznagel}

Directeur de l'Institut für Informations-, Telekommunikations- und Medienrecht auprès de l'Université de Münster

Né en 1957 à Lehrte près de Hanovre. Etudes de droit et de sociologie à Berlin, puis cursus Master of Laws Program de l'Université McGill de Montréal. Thèse soutenue en 1990. 1988-91 : auditeur de justice. 1991-95 : maître de conférences au Département d'études juridiques II de l’Université de Hambourg. 1996 : habilitation et délégation sur une chaire de droit public et administratif à l'Université de Potsdam. 1997 : titulaire de la Chaire de droit public et administratif à la Westfälische Wilhelms-Universität de Münster. Directeur de 1'Institut für Informations-, Telekommunikations- und Medienrecht, Département droit public, auprès de l'Université de Münster. 


\section{Isabell Hülsen}

Journaliste médias au Financial Times Deutschland

Née en 1973 à Düsseldorf. Scolarité à Gummersbach et Hambourg. Etudes de sciences politiques, de journalisme et d'histoire à Hambourg et Paris. Durant les études, stages effectués notamment à Gruner + Jahr/Prisma Presse (Paris). Formation professionnelle de journaliste au Financial Times Deutschland, puis journaliste dans le même quotidien. Depuis 2005, journaliste médias. 2005 : admission à la FT School of Excellence. Depuis 2007, journaliste à Der Spiegel (Hambourg).

\section{Hans-Jürgen Jakobs}

Directeur de la rubrique Médias du quotidien Süddeutsche Zeitung (Munich)

Né en 1956 à Wiesbaden. Etudes en sciences économiques à Mayence. 1985-86 : Formation professionnelle de journaliste dans la rédaction des quotidiens Mainzer Allgemeine Zeitung/Wiesbadener Tageblatt. 1986-89: journaliste au magazine $Z V+V$ et rédacteur en chef du magazine Copy (groupe Handelsblatt, Düsseldorf). 1990-93 : directeur de la rubrique Economie du quotidien Münchner Abendzeitung. 1993-2001 : journaliste à l'hebdomadaire Der Spiegel (Hambourg). Depuis 2001, directeur de la rubrique Médias du quotidien Süddeutsche Zeitung (Munich).

\section{Uwe Kammann}

Directeur de l'Institut Adolf-Grimme (Marl)

Né en 1948 à Bünde. Etudes de germanistique et de romanistique. Journaliste au quotidien Rheinische Post (Düsseldorf). 1978 : journaliste à l'agence Evangelischer Pressedienst (epd). 1984 : responsable de la rédaction du bulletin d'information spécialisé epd medien. Nombreuses contributions à la presse quotidienne et hebdomadaire, émissions radio et $\mathrm{TV}$, conférences et animations de débats (congrès professionnels). Membre de divers jurys, dont: Prix Adolf-Grimme, Deutscher Fernsehpreis, Hörspielpreis der Kriegsblinden. Membre du comité consultatif du symposium annuel Mainzer Tage der Fernsehkritik et de la Filmakademie Baden-Württemberg. Distinctions : Deutscher Preis für Medienpublizistik et Hans-Bausch-Media-Preis.

\section{Thomas Kleist}

Avocat, ancien Secrétaire d'Etat

Né en 1955. Etudes de droit à l'Université de Sarre. 1980-93 : maître de conférences. 1985 : Directeur et président du directoire de la Landesanstalt für das Rundfunkwesen des Saarlandes, SLM (autorité de régulation du secteur privé des médias du Land de Sarre). 1994-96: Président de la Direktorenkonferenz der Landesmedienanstalten (DLM : conférence permanente des directeurs des autorités de régulation des médias des Länder). 1996-99 : Secrétaire d'Etat au gouvernement du Land de Bavière. Depuis 2000 : avocat et activités de conseil en management (médias, secteur hospitalier). 
Depuis 2000 : Directeur de l'Institut du Droit européen des médias (EMR, Sarrebruck/Bruxelles). Président du Conseil d'administration du Saarländischer Rundfunk (établissement de radiodiffusion de droit public de la Sarre). Vice-président du conseil de surveillance des sociétés Werbefunk GmbH et Telefilm GmbH. Membre du conseil de surveillance de Radio Salü GmbH. Président du comité consultatif de la société OBG $\mathrm{GmbH}$. Membre du cercle des managers de la Fondation Friedrich-Ebert. Nombreuses publications sur les médias.

\section{Jasmin Kundan}

Assesseur (jusqu'en juin 2008) à la 6ème section de décision du Bundeskartellamt (Medias)

1990-95 : Etudes de droit à l'Université de Heidelberg, puis de Cologne ; premier Examen d'Etat. 1996-1998 : Stage préparatoire juridique à Düsseldorf ; deuxième Examen d'Etat. Juillet 1998-août 2001 : Conseiller rapporteur à l'Autorité de régulation des télécommunications et des services postaux (ex-RegTP, actuellement Bundesnetzagentur) ; procédures d'autorisation ex ante concernant la rétribution des prestations exécutées d'avance par Deutsche Telekom AG. Août 2001-mai 2008 : Assesseur à l'Office fédéral des cartels, d'abord à la $11^{\text {ème }}$ Section de décision : Contrôle des abus sur les marchés de l'électricité jusqu'en mai 2004, puis à la $6^{\text {ème }}$ Section de décision : Médias, sport, industrie du papier (contrôle des fusions et procédures en matière de cartels). Depuis juin 2008: Expert détaché auprès de la Commission Européenne de Bruxelles, Direction Générale de la concurrence (Merger Network).

\section{Alain Lancelot \\ Ancien membre du Conseil constitutionnel ; président de la commission chargée d'examiner les problèmes de concentration dans les médias}

Né en 1937 à Chêne-Bougeries (Suisse). Professeur des Universités, Docteur ès lettres et sciences humaines, docteur en études politiques, diplômé de l'Institut d'études politiques de Paris. 1987-1996 : Directeur de l'Institut d'études politiques de Paris. Administrateur de la Fondation Nationale des Sciences Politiques, membre du Conseil d'administration de l'ENA. 1992-1993 : membre de la Commission de réforme du mode de scrutin et du Comité consultatif pour la révision de la Constitution. 1996-2001 : membre du Conseil Constitutionnel.

\section{Joseph Lorent}

Grand reporter au quotidien Luxemburger Wort et membre du Conseil de direction

Né en 1946. Journaliste au quotidien Luxemburger Wort depuis 1973 : d'abord directeur de la rubrique Politique et affaires sociales, parallèlement responsable du dossier Médias ; aujourd'hui, Grand reporter et membre du Conseil de direction. Avril-mai 1994 : observateur désigné par l'UE pour les premières élections libres en Afrique du Sud. Depuis 1981, membre du Conseil Luxembourgeois de la Presse, dans diverses fonctions : secrétaire général de 1981 à 1994, et depuis 2004 ; président de 1994 à 1996 
et de 2002 à 2004. Ancien président de l'Union des Journalistes Luxembourg (UJL). Membre de la Commission de Conseil sur les médias auprès du Premier ministre.

\section{Jean-Louis Missika}

Consultant (stratégie médias et conseil en communication) et professeur à Sciences Po, Paris.

Diplômé en philosophie, sciences politiques, gestion et sciences économiques. 19791984 : conseiller du Président Directeur Général d'Antenne 2. 1988-1991 : chef du service information et diffusion du Premier ministre Michel Rocard. 1991-1995 : collaborateur de la Sofres. 1995-1998 : Directeur général de l'institut de sondages BVA. Puis, création et direction de la société JLM Conseil (conseil en stratégie médias et en communication). Depuis 1984 : professeur de sociologie des médias à l'Institut d'études politiques de Paris. Auteur de nombreuses publications, notamment de La fin de la télévision (2006).

\section{Bénédicte de Peretti}

Correspondante en Allemagne du quotidien économique La Tribune.

Née en 1959 à Versailles. Formation commerciale. Diplômée de l'ESCP-EAP European School of Management in Paris, Oxford et Düsseldorf (Diplom-Kauffrau). 1982-1988 : journaliste à Paris dans différents quotidiens (La Croix et Libération). De 1988 à juin 2008 : correspondante en Allemagne du quotidien économique La Tribune

\section{Jacques Rigaud}

Ancien Président directeur général de RTL radio (Paris). Vice-président de la Fondation Robert Schuman (Paris).

Né à Paris en 1932. Diplômé de l'ENA. 1958-1985 : professeur à l'Institut d'études politiques de Paris. 1969-1973 : directeur du cabinet de Jacques Duhamel, ministre de l'Agriculture, puis des Affaires culturelles. 1975-1978: Sous-directeur général de l'UNESCO ; puis conseiller spécial du ministre des Affaires étrangères Jean FrançoisPoncet. 1980-juin 2000 : administrateur délégué de la Compagnie luxembourgeoise de Télédiffusion (CLT, Luxembourg) et Président directeur général de RTL radio (Paris). 1981-1986 : responsable de la construction du musée d'Orsay, auteur de plusieurs rapports au gouvernement sur la politique culturelle. Depuis 1979 : président de l'Association pour le développement du mécénat industriel et commercial (ADMICAL), fondée la même année. Depuis 1985 : membre du Conseil d'administration de la Fondation nationale des sciences politiques, de l'Alliance française, de l'Institut national de l'Audiovisuel (INA) et du Festival de Cannes. Auteur de nombreux ouvrages, notamment «La culture pour vivre » (1975), « Libre culture » (1990), «L'exception culturelle » (1995), «Les deniers du rêve - essai sur l'avenir des politiques culturelles » (2001) et «Vivre à propos » (2005). 


\section{Prof. Dieter Schmidtchen}

Titulaire de la chaire d'économie nationale (politique économique) de l'Université de la Sarre, Directeur du Centre de recherche sur l'analyse économique du droit

Né en 1940 à Schwiebus, Mark Brandenburg. Etudes de sciences économiques à la Phillips-Universität de Marburg, doctorat (1971), puis habilitation en sciences économiques (1976). 1977-78 : professeur à l'Université de Cologne. 1979 : nommé à l'Université de la Sarre sur la chaire d'économie nationale (politique économique); depuis 1979 également Directeur du Centre de recherche sur l'analyse économique du droit de l'Université de la Sarre.

\section{Prof. Norbert Schneider}

Président de la Landesanstalt für Medien Nordrhein-Westfalen (LFM : autorité de régulation du secteur privé des médias du Land de Rhénanie du Nord-Westphalie)

Né en 1940 à Langenau (Wurtemberg). 1959-64 : études de théologie protestante et de journalisme à Tübingen, Marburg et Hambourg. 1965-71 : activités de recherche, formation au ministère pastoral, formation professionnelle de journalisme (Südwestfunk : établissement de radiodiffusion de droit public commun à la Rhénanie-Palatinat et au nord du Bade-Wurtemberg). 1971-81 collaborateur, puis directeur du Gemeinschaftswerk der Evangelischen Publizistik (Francfort/Main). 1981-86 : Directeur des programmes (radio et TV) du Sender Freies Berlin (établissement de radiodiffusion de droit public du Land de Berlin). 1986-93: Directeur de la maison de production Allianz-Film GmbH (Berlin). Depuis 1993 : Directeur de la LfM (Düsseldorf). 1999-2003 : Président de la Direktorenkonferenz der Landesmedienanstalten (DLM : conférence permanente des directeurs des autorités de régulation des médias des Länder). Depuis 2003 : Président de la Cellule commune des Landesmedienanstalten pour les questions relatives aux programmes, à la publicité et à la compétence médiatique. Nombreuses publications sur la radio, la TV et les médias. Edition de publications spécialisées. Membre de diverses instances et commissions dans les médias, notamment : Mahrenholz-Kommission, conseil de surveillance de l'Institut Adolf-Grimme (président), Commission des aides de la Filmstiftung NRW, comité consultatif du symposium annuel Mainzer Tage der Fernsehkritik, Jury du Fernsehfilm-Festival Baden-Baden (président). 2004 : élevé au titre de Professeur des universités par le gouvernement du Land de Rhénanie du Nord-Westphalie.

\section{Prof. Werner Schwaderlapp}

Ancien président de Endemol Deutschland, Directeur du me:mi Medien+Entertainment Management Institut auprès de la Europa FH, Cologne

Né en 1950. Etudes de philosophie, de journalisme et d'économie à Francfort, Tubingen et Mayence ; Advanced Management Programm de la Harvard Business School. 197882 : Conseiller technique auprès du directeur des programmes de la seconde chaîne publique Zweites Deutsches Fernsehen (ZDF). 1982-87 : Création et direction de la Direction du développement de la ZDF. 1987-93 : Directeur de la planification des programmes de la ZDF. 1993-97 : président directeur général de ZDF Enterprises GmbH. 
1998-2000 : Directeur du développement de Endemol Deutschland. 1999-2000 : Président du conseil de surveillance de Helkon Media AG (filiale de Endemol Deutschland). 2000-01 : Président de Endemol Entertainment Productions. 2001-mai 2003 : Président de Endemol Deutschland Holding. 2003 : Création du cabinet de conseil en management et communication ws-mediaconsult. 1997-82 : chargé de cours à l'Institut für Publizistik de l'Université de Mayence. 1998-2001 : chargé de cours (management culturel et médias) à la Hochschule für Musik und Theater de Hambourg. Depuis 2002 : titulaire de la chaire de management des médias à la Europa Fachhochschule Fresenius, Cologne. 2003 : Cofondateur de la Hochschule für Wirtschaft und Medien. Membre du Conseil d'orientation de la Europa Fachhochschule Fresenius. Fondateur et directeur du me:mi Medien+Entertainment Management Institut. Membre de la Commission médias de la Chambre de commerce et d'industrie de Cologne. Membre de l'International Academy for Television Arts and Sciences, New York.

\section{Andreas Stopp}

Directeur de la rédaction "Médias et société » de la radio nationale allemande Deutschlandfunk (Cologne)

Né en 1958. Etudes de didactique des médias à Bonn et Cologne. Journaliste free lance et enseignant/chercheur à l'Université de Bonn. Actuellement, notamment chargé de cours (journalisme) à l'Université de Bamberg. Responsable de la rédaction «médias » et de la rédaction « voyages » à la radio publique Deutschlandfunk (Cologne). 


\section{INDICATIONS BIBLIOGRAPHIQUES}

\section{OUVRAGES GENERAUX ET QUESTIONS GENERALES}

Balle F., Médias et société, $13^{\mathrm{e}}$ édition, Montchrestien, Paris, 2007

Balle F., Cohen-Tanugi L., Dictionnaire du web, Dalloz, Paris, 2001

BAUSCH H. (dir.), Rundfunk in Deutschland, DTV, Munich, 1980

BoURGEOIS I., «Médias français et allemands. Convergences et divergences dans le contexte européen », Revue d'Allemagne, $\mathrm{n}^{\circ}$ 1/2005

BURKarT R., Kommunikationswissenschaft, $4^{\mathrm{e}}$ édition, UTB, Vienne/Cologne/Weimar, 2002

DörR D., Müller-GrafF P.-C. (eds), Medien in der Europäischen Gemeinschaft, Nomos, Baden-Baden, 2007

HENLE V., Fernsehen in Europa. Strukturen, Programme und Hintergründe, Kopäd, Munich, 2001

Mattelart A., Histoire de la société de l'information, Editions La Découverte, $3^{\mathrm{e}}$ édition, Paris, 2006

MEYN H., Massenmedien in Deutschland, réédition, UVK Verlagsgesellschaft, Constance, 2004

Missika J.-L., La fin de la télévision, Editions du Seuil, Paris, 2006

Missika J.-L., Wolton D., La Folle du logis, la télévision dans les sociétés démocratiques, Gallimard, Paris, 1984

RIGAUD J., L'Exception culturelle - Culture et pouvoirs sous la $V^{e}$ République, Grasset et Fasquelle, Paris, 1995

SCHWARZKOPF D. (ed), Rundfunkpolitik in Deutschland, DTV, Munich, 1999

\section{DROIT DES MÉDIAS}

Berger E. G., Schalast C. (eds), Telekommunikations- und Medienrecht, Nomos, Baden-Baden, 2007

Dörr D., SchWARTMann R., Medienrecht, Hürtig Jehle Rehm, Heidelberg, 2006

DERIEUX E., Droit des médias, Dalloz, Paris, 2005

DerieuX E., Granchet A., Droit des médias : Droit français, européen et international, LGDJ, 5e édition, Paris, 2008

EMR, Der Rechtsrahmen für die neue Medienlandschaft. Eine Richtlinie über audiovisuelle Mediendienste, Sarrebruck, 2007 (www.emr-sb.de/home/schriftr.htm)

Petersen J., Medienrecht und Informationsrecht, Nomos, Baden-Baden, 2005

RosSnAGEL A. (ed), Neuordnung des Medienrechts. Neuer rechtlicher Rahmen für eine konvergente Technik? Nomos, Baden-Baden, 2005 


\section{CONVERGENCE}

BALLE F., «La convergence de l'audiovisuel, de l'informatique et des télécommunications : mythes et réalités », in Clés pour le siècle, Dalloz, Paris, 2000

Dimitrakopoulou K., Medienkonvergenz, und der Relevante Produktmarkt in der europäischen Fusionskontrolle, Nomos, Baden-Baden, 2007

JAKOBS H.-J., LANGENBUCHER W. R. (ed), Das Gewissen ihrer Zeit : Fünfzig Vorbilder des Journalismus, Picus Verlag, Vienne, 2004

KLeist T., LAMPRECht-Weißenborn N., Scheuer A., Audiovisuelle Mediendienste heute und morgen Die Revision der EG-Fernsehrichtlinie, Friedrich-Ebert-Stiftung, Berlin, 2007

\section{TÉLÉCOMMUNICATIONS}

BourgeoIS I., «Télécommunications - Quelle régulation à l'heure de la convergence ? », Regards sur l'économie allemande, $\mathrm{n}^{\circ}$ 78/2006

Holznagel B., Enaux C., Nienhaus C., Telekommunikationsrecht, $2^{\mathrm{e}}$ édition, C.H. Beck Verlag, Munich, 2006

\section{QUESTIONS DE RÉGULATION}

Gedanken zu den Medien und ihrer Ordnung. Festschrift für Dr. Victor Henle, Schriftenreihe des Instituts für Europäisches Medienrecht, Nomos, Baden-Baden, 2007

HENLE V., Das gesellschaftsplurale Ordnungs- und Kontrollmodell des deutschen Rundfunks. Überlegungen zu einer Reform, Arbeitspapier $n^{\circ} 12$, Hans-Bredow-Institut an der Universität Hamburg, juin 2002

Holznagel B., DöRR D., Hildebrand D., Elektronische Medien - Entwicklung und Regulierungsbedarf, Münchner Kreis, 2008 (www.muenchner-kreis.de)

Kleist T., Henle V., DörR D., Kopp R., Closs W., Die Landesmedienanstalten (eds), Die Rechtsstellung der Landesmedienanstalten in grenzüberschreitenden Angelegenheiten, Vistas, Berlin, 1996

Kleist T., Roßnagel A., Scheuer A., Die Reform der Regulierung elektronischer Medien in Europa. Dargestellt am Beispiel der EG, Belgiens, Deutschlands, Frankreichs, Italiens und des Vereinigten Königreichs, Coll. Medienforschung der Landesanstalt für Medien NRW (LfM), vol. 53,Vistas, Berlin, 2007

\section{ECONOMIE ET MARCHE DES MEDIAS}

BöGE U., «Effizienz und Wettbewerb aus Sicht des Bundeskartellamts », in OBERENDER P. (ed), Effizienz und Wettbewerb, Duncker \& Humblot, Berlin, 2005

BoURGEOIS I., «Springer/ProSiebenSat.1 : quel droit de la concurrence ? », Regards sur l'économie allemande, $\mathrm{n}^{\circ} 75 / 2006$ 
BoURGEOIS I., Radios et télévisions privées en Allemagne. Entre la loi et le marché, Coll. Travaux et Documents du CIRAC, Levallois-Perret, 1995

Christiansen O., Gemeinsame Beschaffung und Wettbewerb: Einkaufskooperationen im EG-Kartellrecht, Peter Lang, Berne, 2003

HEINRICH J., « Pressefusionskontrolle«, in MedienWirtschaft, vol. 4, 2004

Henle V., Paetow K., KLEemann D., Konzentrationskontrolle im Rundfunk und wettbewerbliche Fusionskontrolle, Vistas, Berlin, 2001

SCHMIDTCHEN D., «Effizienz als Leitbild der Wettbewerbspolitik: Für einen 'more economic approach' », in OBERENDER P., Effizienz und Wettbewerb, Duncker \& Humblot, Berlin, 2005

SCHMidTChen D., Cooter, R. (eds), Constitutional Law and Economics of the European Union, Edward Elgar, 1997

SCHMidTChen D., SCHMIDT-Trenz H.-J. (eds), Vom Hoheitsstaat zum Konsensualstaat. Ökonomische Analyse der Flexibilisierung von Genehmigungsverfahren, Nomos, Baden-Baden,1999

\section{OPINION ET PLURALISME}

BoURGEOIS I., «Opinions et valeurs : 60 ans de démoscopie allemande », Regards sur l'économie allemande, $\mathrm{n}^{\circ}$ 80/2007

Dörr D., Stockmann K., HaEckel H., Henle V., Lange B. P., Engel C., Kübler F., DiE LANDESMEDIENANSTALTEN (eds), Die Sicherung der Meinungsvielfalt, Vistas, Berlin, 2001

FEISE C., Medienfreiheit und Medienvielfalt gemäß Art. 11 Abs. 2 der Europäischen Grundrechtecharta, Nomos, Baden-Baden, 2005

Holznagel B., Ricke T., SiMON I. M., Mediennutzerschutz : Beschwerderechte für Fernsehen, Hörfunk und Internet, LfM, Düsseldorf, 2008

Kontrolle von Meinungsmacht in Zeiten der Konvergenz - Demokratie und Wirtschaft brauchen klare Regeln, Friedrich-Ebert-Stiftung, Berlin, séminaire, 6 novembre 2006 (www.fes.de)

\section{SERVICE PUBLIC}

BDI, „Dritte Säule“ des öffentlich-rechtlichen Rundfunks im Internet nicht gerechtfertigt. Positionspapier zu den Beratungen des 12. RÄndStV, 20-05-2008 (www.bdi.eu)

BoURgeOIS I., Radio et télévision publiques en Allemagne. Un modèle à l'épreuve de la nouvelle Europe, Coll. Travaux et Documents du CIRAC, Paris, 1993

DöRR D., «Eine Chance. ARD und ZDF sollten den Drei-Stufen-Test ernst nehmen », epd medien, $\mathrm{n}^{\circ} 34 / 2008$

EBERLE C.-E., «Der öffentlich-rechtliche Funktionsauftrag im Internet », epd medien, $n^{\circ} 47 / 2008$

HEINRICH J., «Zur Funktionalität des Wettbewerbs im dualen System », in RIDDER C.- 
M. (ed.), Bausteine einer Theorie des öffentlich-rechtlichen Rundfunks, VS Verlag, Wiesbaden, 2005

HENLE V., «Comment limiter l'expansion de l'audiovisuel public allemand? », Regards sur l'économie allemande, $\mathrm{n}^{\circ} 87 / 2008$

Henle V., «Wie testet man Public Value ? Ein vergleichender Blick auf das britische Verfahren », epd medien, $n^{\circ}$ 92/2007

KLEIST T., SCHEUER A., «Klärung von Grundsatzfragen. Die EU überprüft die Finanzierung des öffentlich-rechtlichen Rundfunks », in Funkkorrespondenz, n 10/2005

LIBERTUS M., «Die Revision des EU-Regulierungsrahmens für elektronische Kommunikation. Problematik aus Sicht des öffentlich-rechtlichen Rundfunks », Media Perspektiven, $\mathrm{n}^{\circ} 5 / 2008$

SCHWADERLAPP W., HeYgSTER A. (ed), Vielfalt und Wettbewerb der Programme. Konkurrenz, Kontrast, Koordination im Fernsehen, Hase \& Kœhler, Mayence, 1980

\section{RAPPORTS ET DOCUMENTS OFFICIELS}

ALM-Jahrbuch 2007, Vistas, Berlin, 2008

ALM, GSDZ (eds), Digitalisierungsbericht 2006, Vistas, Berlin, 2006

BundeSKARTEllamt, 6. Beschlußabteilung, Stellungnahme des Vorsitzenden, Klaus Paetow zum Gutachten von Prof. Hasebrinck auf Anfrage von Prof. Dörr, 01-07-2005

Crossmediale Verflechtungen als Herausforderung für die Konzentrationskontrolle. Bericht der Kommission zur Ermittlung der Konzentration im Medienbereich (KEK) über die Entwicklung der Konzentration und über Maßnahmen zur Sicherung der Meinungsvielfalt im privaten Rundfunk, Vistas, Berlin, 2007

DeutSCHER BundeSTAg, Schlussbericht der Enquête-Kommission „Kultur in Deutschland", décembre 2007 (http://dip.bundestag.de/btd/16/070/1607000.pdf)

HASEBRINCK U., Zur Berücksichtigung medienrelevanter verwandter Märkte bei der Anwendung des Zuschaueranteilsmodells (\$26 Abs. 2 Satz 2 RStV). Kommunikationswissenschaftliches Gutachten für die KEK, Hans-Bredow-Institut, Hambourg, 2003

HolzNaGeL B., Rechtsgutachten zur Auslegung des §26 Abs. 2 Satz 2,2. Alt. RStV, juillet 2005

KLeist T., Rossnagel A., Scheuer A., Stellungnahme des EMR im Rahmen der öffentlichen Befragung durch die KEK, Berücksichtigung medienrelevandter verwandter Märkte im Rahmen der Medienkonzentrationsrechtlichen Prüfung (\$26 Abs. 2 Satz 2,2. Alt. RfStV), EMR, juin 2005

Mitteilung der Bundesregierung an die Europäische Kommission. Staatliche Beihilfen E 3/2005. Die Finanzierung der öffentlich-rechtlichen Rundfunkanstalten - Deutschland, in epd medien, $\mathrm{n}^{\circ} 38 / 2005$

Mitteilung der Generaldirektion Wettbewerb der EU-Kommission zur Finanzierung des öfentlich-rechtlichen Rundfunks in Deutschland, Bruxelles, mars 2005, in Funkkorrespondenz, $\mathrm{n}^{\circ} 10 / 2005$

MONOPOLKOMMISSION, Wettbewerbsentwicklung bei der Telekommunikation 2007: Wendepunkt der Regulierung, Sondergutachten n ${ }^{\circ}$ 50, 2007 
MonOPOLKOMMission, Zur Gesamtbeurteilung des Meinungseinflusses eines Unternehmens gemäß § 26 Abs. 2 Satz 2,2 Alternative des Rundfunkstaatsvertrags/ Hier: Stellungnahme zum Gutachten von Prof. Dr. Uwe Hasebrinck, Bonn, 2005

Monopolkommission, Die Pressefusionskontrolle in der Siebten GWB-Novelle, Sondergutachten $\mathrm{n}^{\circ} 42,2004$

MONOPOLKOMMISSION, Wettbewerbspolitik im Schatten "Nationaler Champions", Hauptgutachten XV, 2002/2003

RAPPORT AU PREMIER MINISTRE sur les problèmes de concentration dans le domaine des médias (Rapport de la commission présidée par Alain Lancelot), Paris, décembre 2005

SENAT, Rapport d'information fait au nom de la commission des Affaires économiques sur le bilan et les perspectives d'évolution de l'ARCEP, Sénat, Paris, juin 2007

Vielfalt durch privaten Rundfunk-Eckdaten einer Leistungsbilanz, Gutachten im Auftrag des VPRT, Goldmedia, Berlin, 2008

WISSENSCHAFTLICHER BEIRAT BEIM BUNDESMINISTERIUM FÜR WIRTSCHAFT UND TECHNOLOGIE, Reform der europäischen Kartellpolitik, juillet 2000

WISSENSCHAFTLICHER BEIRAT BEIM BUNDESMINISTERIUM FÜR WIRTSCHAFT UND TEChNOLOGIE, „, Offene Medienordnung “, Gutachten, octobre 1999

\section{REVUES}

epd medien (www.epd.de)

Funkkorrespondenz (www.funkkorrespondenz.de)

Media-Perspektiven (www.media

Revue européenne des médias, Institut de Recherche de l'European Business School, Institut de Recherche et d'Etudes sur la Communication (IREC), Université PanthéonAssas Paris 2

\section{SITES}

www.arcep.fr

www.bundeskartellamt.de

www.bundesverfassungsgericht.de

www.cirac.u-cergy.fr

www.csa.fr

WwW.conseil-concurrence.fr

www.ddm.gouv.fr

www.europa.eu

www.kek-online.de

www.lfm-nrw.de

www.monopolkommission.de

www.tlm.de 



\section{OUVRAGES PARUS AUX EDITIONS DU CIRAC}

\section{- dans la collection Travaux et documents du CIRAC :}

\section{Isabelle BOURGEOIS (dir.), Allemagne : compétitivité et dynamiques territoriales.}

La compétitivité de l'économie allemande ne repose pas uniquement sur la puissance, la spécialisation sectorielle et la tradition exportatrice de son industrie, mais également sur la qualité de ses infrastructures et l'excellence d'une gestion territoriale fondée sur l'autonomie régionale et le polycentrisme. Dans le cadre du fédéralisme, c'est au niveau des Länder que, pour l'essentiel, se conçoit, se décide et se construit la dynamique des territoires et que s'affirme ainsi la capacité de l'Allemagne à faire face aux mutations structurelles d'une économie hautement développée et largement ouverte sur le monde.

La vitalité et la solidité de l'économie allemande sont dans une large mesure la résultante de dynamiques territoriales plurielles, dont cet ouvrage montre, à travers une série de portraits des Länder les plus significatifs, qu'elles sont elles-mêmes les fruits de choix politiques et économiques régionaux assumés. Ainsi de la Bavière agricole devenue site high-tech, du Bade-Wurtemberg qui a construit son identité sur la culture industrieuse de ses habitants, de la Rhénanie du Nord-Westphalie, ancien 'pays noir' reconverti dans le multimédia et la logistique intermodale, de la Saxe qui, une fois affranchie de l'économie planifiée de l'ex-RDA, redevient un site industriel de premier plan au centre de la nouvelle Europe...

Pourtant, ce modèle de gestion territoriale polycentrique est lui-même confronté à l'épreuve du changement : la globalisation des activités et, plus encore, l'accélération de l'intégration européenne, placent aujourd'hui l'Allemagne face à un défi de gouvernance. Comment préserver l'autonomie des Länder, garante d'une concurrence régulée des particularismes au sein d'un fédéralisme coopératif, alors que «Bruxelles » tend à piloter les politiques structurelles jusqu'à l'échelon local ? Comment préserver institutionnellement, dans ce processus d'uniformisation, cette diversité régionale qui fonde la prospérité et l'unité de l'Allemagne?

Juillet 2007, 144 p., ISBN 978-2-905518-35-4 (25€).

Leo Kißler, René Lasserre, Marie-Hélène PaUtrat (dir.), Modernisation des services publics et management social en France et en Allemagne.

Consolidation des budgets publics, mise en concurrence et dérégulation des services d'intérêt général à l'échelle européenne, évolution des attentes des usagers-clients au regard de la flexibilité et de la qualité des services rendus, nécessité de promouvoir à l'échelon des Etats et des régions un environnement public stimulant pour l'activité économique... Ces changements imposent une rénovation des modèles classiques d'organisation de l'action publique et la mise en œuvre de nouvelles formes de management des ressources humaines.

Cet ouvrage rassemble les contributions de chercheurs et responsables français et allemands portant sur la gestion de l'emploi et des compétences, la représentation du personnel et les relations sociales dans la sphère publique. Il en ressort un tableau différencié qui met en évidence les conditions et les modalités très variables selon lesquelles ces grands enjeux de la modernisation de l'Etat sont abordés de part et d'autre du Rhin.

Juillet 2007, 144 p., ISBN 978-2-905518-34-0 (20 €).

\section{Isabelle BOURGEOIS (dir.), Le modèle social allemand en mutation.}

Le modèle social allemand est en crise. Depuis 2003, gouvernement et partenaires sociaux ont commencé à le réformer : révision du système d'indemnisation chômage, revalorisation de l'apport individuel à l'assurancemaladie ou aux régimes de retraite, nouvelle approche globale de la politique salariale pour concilier compétitivité des entreprises et solidarité collective. Mais l'Allemagne doit aussi et surtout refonder son contrat social 
et trouver un nouvel équilibre entre le marché et la solidarité, entre la promotion de l'activité et la redistribution, entre les droits et devoirs respectifs de l'individu et de la collectivité.

Les réformes en cours impliquent aussi un débat sur les valeurs qui fonderont les nouveaux choix de société allemands et assureront le maintien de la performance économique du pays dans le cadre d'une globalisation assumée et d'un engagement européen réaffirmé. C'est dans la perspective d'une Europe compétitive dans l'économie du savoir que l'Allemagne inscrit la refondation de son modèle économique et social.

Septembre 2005, 208 p., ISBN 2-905518-33-2 (25€).

\section{Hervé Joly (dir.), Formation des élites en France et en Allemagne.}

Si de nombreux travaux récents abordent les élites en général, seules quelques initiatives isolées analysent, sur des aspects particuliers, les élites françaises actuelles. A l'inverse, en Allemagne, après une longue période d'occultation liée à des associations historiques embarrassantes, les publications sociologiques sur les élites foisonnent aujourd'hui de manière spectaculaire. Cette profusion traduit cependant plus une proclamation d'intérêt pour un concept qu'une restitution massive de recherches empiriques nouvelles.

Publier des textes d'auteurs français et allemands sur ce thème constitue donc une démarche inédite, développée en trois temps dans cet ouvrage : étude des conditions dans lesquelles se sont construites les différences observées dans la formation des élites des deux pays, présentation des enquêtes récentes sur le recrutement des élites politiques, administratives et économiques ; analyse de la manière dont, en pratique, les différences constatées se répercutent sur les conditions de travail en commun dans les diverses entreprises mixtes constituées depuis quelques années.

Juillet 2005, 228 p., ISBN 2-925518-32-4 (20€).

\section{Patricia Commun (dir.), L'ordolibéralisme allemand. Aux sources de l'économie sociale de marché.}

L'ordolibéralisme a retrouvé une actualité scientifique. Il est considéré aujourd'hui comme un précurseur de l'institutionnalisme et, plus largement, d'un retour à une économie culturelle. L'ordolibéralisme, comme toute pensée économique, repose en effet sur des postulats philosophiques et s'inscrit dans un cadre éthique et culturel. C'est cette dimension philosophique et culturelle de l'ordolibéralisme allemand qui est analysée dans cet ouvrage collectif par des germanistes, des économistes et des philosophes français, anglais et allemands. Le livre aborde également la question de l'application politique de cette pensée économique, en apportant des éclairages sur certains des principes mis en œuvre, ainsi que sur l'action politique et idéologique entreprise par les ordolibéraux eux-mêmes en RFA dans les années d'après-guerre.

Juin 2003, 274 p., ISBN 2-905518-31-6 (32€).

\section{Isabelle BOURGEOIS (dir.), Allemagne 2001. Regards sur une économie en mutation.}

Confronté au double défi de l'unification et de la mondialisation, le «modèle rhénan » semblait s'être englué dans ses pesanteurs et ses rigidités. Pourtant, le gouvernement Schröder est parvenu à sortir l'Allemagne de sa torpeur. Une nouvelle dynamique collective de modernisation a saisi pouvoirs publics, acteurs économiques, partenaires sociaux. Elle se fonde sur une acceptation raisonnée du changement. Mais le défi que relève la société allemande dépasse l'horizon mental national : c'est avant tout un choix européen assumé. Parce que le site Allemagne est au cœur de l'Europe, il ne peut échapper à la modernisation : de son dynamisme retrouvé dépendra la compétitivité de l'espace économique européen dans son ensemble, donc celle de chacun des Etats membres. En révélant le haut degré d'interdépendance des économies nationales au sein de la zone euro, l'avènement de la monnaie unique en a forcé la prise de conscience collective...

Juin 2001, 274 p., ISBN 2-905518-30-8 (32 €).

Jacques PATEAU, Une étrange alchimie. La dimension interculturelle dans la coopération francoallemande.

A travers des centaines d'interviews réalisées dans des entreprises appartenant aux secteurs les plus variés, l'auteur nous fait découvrir non seulement les différences dans les manières d'organiser le travail, de gérer ou 
de communiquer, mais surtout l'impact concret des développements historiques qui ont façonné nos cultures. Ce livre recèle des outils précieux pour comprendre la cohérence et l'intelligence de chaque système.

5 e édition, novembre 2005, 254 p., ISBN 2-905518-29-4 (32€).

Rémi LALLEMENT, L'unification sans miracle. L'économie allemande en mutation (1990-1995).

L'unification révèle les limites du «modèle allemand»: elle absorbe une bonne part des ressources nationales, exacerbe les conflits de répartition et, surtout, bat en brèche sa compétitivité internationale. En réponse à ces chocs, l'Allemagne s'est engagée dans un vaste aggiornamento en de nombreux domaines : des pratiques managériales aux conventions collectives, en passant par la politique industrielle. L'unification n'est pas seulement une épreuve de vérité, mais bel et bien l'occasion de renforcer les atouts de l'économie allemande en les adaptant aux exigences nouvelles de la compétition mondiale.

Octobre 1995, 176 p., ISBN 2-905518-28-6 (18,29€)

- dans la collection Deutsch-französische Studien zur Industriegesellschaft aux éditions Campus (Francfort/New York) :

Leo KIBLER, René LASSERRE, Marie-Hélène PAUTRAT (dir.), Öffentlicher Dienst und Personalmanagement. Zur Verwaltungsreform in Deutschland und Frankreich. 2006, 212 p., ISBN 3-593-38178-8.

Anne-Marie Le GloanneC (ed.), Mehr oder weniger Staat? Die Rolle des Staates in Deutschland nach 1989. 2003, 260 p., ISBN 3-593-37245-2.

Philippe D'IRIBARNE, Ehre, Vertrag, Konsens. Unternehmensmanagement und Nationalkulturen. 2003, 260 p., ISBN 3-593-37245-2.

\section{- autres publications du CIRAC :}

Regards sur l'économie allemande - Bulletin économique du CIRAC

5 numéros par an (abonnement : $200 €)$. ISSN 1156-8992

CIRAC-Forum - Bulletin d'information pour la coopération franco-allemande dans les sciences humaines et sociales

4 numéros par an. Diffusé gratuitement par courrier électronique et consultable sur le site du CIRAC.

Liste complète des publications du CIRAC sur le site : www.cirac.u-cergy.fr 

Composition : CIRAC

CIRAC, c/o Université de Cergy-Pontoise

33 Boulevard du Port - 95011 CERGY-PONTOISE CEDEX

Impression : Dupli-print

2 rue Descartes - Z.I. Sezac - 95330 DOMONT

Dépôt légal : juillet 2008

Imprimé en France 





\title{
LES MÉDIAS À L'ÈRE DU NUMÉRIQUE \\ Réflexions franco-allemandes pour l'Europe
}

Sous la direction d'Isabelle Bourgeois

La télévision est en sursis. Bientôt, elle ne sera plus le seul média de référence : inexorablement, Internet poursuit sa montée en puissance, et le portable se métamorphose en téléviseur. La numérisation a brouillé les frontières entre les divers moyens de communication électroniques : audiovisuel et télécommunications convergent vers un nouvel ensemble aux contours indéfinis.

Comment l'Europe réagit-elle à ces mutations ? Comment en particulier la France et l'Allemagne abordent-elles ces défis? Malgré d'importantes différences dans l'organisation concrète des marchés et de leur régulation, nos deux pays partagent foncièrement la même approche de la mission de l'audiovisuel, fondée sur la nécessité de garantir le plẹralisme des opinions. Mais pourra-t-il encore assurer cette mission, indispensable au lien social, à l'ère du numérique ? Le consommateur des médias, le citoyen, est aujourd'hui largement libre de ses choix ; faut-il, et si oui, comment, le protéger contre les nouveaux risques susceptibles de naitre dans un avenir médiatique encore incertain ? Face à la convergence de fonctions naguère séparées entre éditeurs, diffuseurs, fournisseurs d'accès, quelle approche faut-il développer pour veiller au libre jeu de la concurrence, au libre accès de tous au marché et à la libre circulation de l'information ? Convient-il de soumettre à l'avenir les médias aux seules lois du marché ? Ou convient-il au contraire de développer un statut d'exception rénové ? Faut-il, à l'ère du numérique, réguler plus, moins, ou autrement ? Et, le cas échéant, où devrait se situer cette régulation : à l'échelon des Etats membres, à celui de l'Europe?

Toutes ces interrogations nous renvoient à la question du sens que nous voulons donner au modèle de société qui naitra des changements que traversent la France, l'Allemagne et l'Union européenne en ce début de l'ère du savoir et de la connaissance. Elles sont au cœur d'un dialogue entre journalistes spécialisés, experts, professionnels et chercheurs réputés, que retrace le présent ouvrage.

René Lasserre

Directeur du CIRAC

Professeur à l’Université de Cergy-Pontoise

\author{
$25 € \mathrm{TTC}$
}

ISBN : 978-2-905518-36-1

\section{CIRAC}

